University of Louisville ThinkIR: The University of Louisville's Institutional Repository

Electronic Theses and Dissertations

$8-2019$

\title{
Cognitive satellite communications and representation learning for streaming and complex graphs.
}

Wenqi Liu

University of Louisville

Follow this and additional works at: https://ir.library.louisville.edu/etd

Part of the Digital Communications and Networking Commons, and the Systems and Communications Commons

\section{Recommended Citation}

Liu, Wenqi, "Cognitive satellite communications and representation learning for streaming and complex graphs." (2019). Electronic Theses and Dissertations. Paper 3272.

https://doi.org/10.18297/etd/3272

This Doctoral Dissertation is brought to you for free and open access by ThinkIR: The University of Louisville's Institutional Repository. It has been accepted for inclusion in Electronic Theses and Dissertations by an authorized administrator of ThinkIR: The University of Louisville's Institutional Repository. This title appears here courtesy of the author, who has retained all other copyrights. For more information, please contact thinkir@louisville.edu. 


\title{
COGNITIVE SATELLITE COMMUNICATIONS AND REPRESENTATION LEARNING FOR STREAMING AND COMPLEX GRAPHS
}

\author{
By \\ Wenqi Liu \\ B.S., Donghua University, 2011 \\ M.S., Stevens Institute of Technology, 2013

\begin{abstract}
A Dissertation
Submitted to the Faculty of the

J. B. Speed School of Engineering of the University of Louisville in Partial Fulfillment of the Requirements for the Degree of
\end{abstract} \\ Doctor of Philosophy in Electrical Engineering \\ Department of Electrical \& Computer Engineering \\ University of Louisville \\ Louisville, Kentucky
}

August 2019 
Copyright 2019 by Wenqi Liu

All rights reserved 

COGNITIVE SATELLITE COMMUNICATIONS AND REPRESENTATION LEARNING FOR STREAMING AND COMPLEX GRAPHS

\author{
By \\ Wenqi Liu \\ B.S., Donghua University, 2011 \\ M.S., Stevens Institute of Technology, 2013
}

A Dissertation Approved On

June 24, 2019

by the following Dissertation Committee:

Hongxiang Li, Dissertation Director

Jacek Zurada

Andre J Faul

Lihui Bai 


\section{DEDICATION}

This dissertation is dedicated to all

who love, support and help human beings to make the earth a better place for us. 


\section{ACKNOWLEDGMENTS}

Firstly, I would like to thank my advisor, Dr. Hongxiang Li, Associate Professor, Department of Electrical and Computer Engineering at the University of Louisville, for his guidance, knowledge and patience during this academic journey.

I also would like to express my appreciation to my committee, Dr. Jacek Zurada, Dr. Andre J Faul, and Dr. Lihui Bai for agreeing to serve on the dissertation committee. I am deeply grateful for their encouragements and suggestions.

I would like to extend my grateful to my supervisor Dr. Bin Xie for his supporting and collaboration during my internship at InfoBeyond Tech.

I would like to acknowledge my colleagues, Chen Cao, Xiaohui Zhang and Ruixuan Han for their supports during the research. At last, special thanks to my family for their great supports, wise counsel and sympathetic ear. Their love is always with me. 


\section{ABSTRACT \\ COGNITIVE SATELLITE COMMUNICATIONS AND REPRESENTATION \\ LEARNING FOR STREAMING AND COMPLEX GRAPHS \\ Wenqi Liu}

June 24, 2019

This dissertation includes two separate topics. The first topic studies a promising dynamic spectrum access algorithm that improves the throughput of satellite communication (SATCOM) under the uncertainty environment. The other topic investigates real-time distributed representation learning for streaming and complex networks.

\section{Cognitive Satellite Communications}

Dynamic spectrum access (DSA) allows a secondary user to access the spectrum holes that are not occupied by primary users. However, DSA is normally operated under uncertainty in a complex SATCOM environment, which could cause more spectrum sensing errors or even service disruption. In this case, DSA requires a decision-making process to optimally determine which channels to sense and access. To this end, I propose a solution that addresses the uncertainty in SATCOM to maximize the system throughput. Specifically, the DSA decision making process is formulated as a Partially Observable Markov Decision Process (POMDP) model. Simulation results prove the effectiveness of our proposed DSA strategy. 


\section{Distributed Real-time Representation Learning of Large Networks}

Large-scale networks have attracted significant amount of attentions to extract and analyze the hidden information from big data. In particular, graph embedding learns the representations of the original network in a lower vector space while maximally preserving the original structural information and the similarity among nodes. I propose a real-time distributed graph embedding algorithm (RTDGE) which is capable of distributively embedding the streaming graph data by combining a novel edge partition approach and an incremental negative sample approach. Furthermore, a real-time distributed streaming data processing platform is prototyped based on Kafka and Storm. On this platform, real-time Twitter network data can be retrieved, partitioned and processed for state-of-art tasks including synonymic user detection, community classification and visualization.

For complex knowledge graphs, existing works cannot capture the complex connection patterns and never consider the impacts from complicated relations, due to the unquantifiable relationships. In this dissertation, a novel hierarchical embedding algorithm is proposed to hierarchically measure the structural similarities and the impacts from relations by constructing a multi-layer graph. Then an advanced representation learning model is designed based on an entity's context, which is generated by taking random walks on the multi-layer content graph. Experimental results show that our proposed model outperforms the state-of-the-art techniques. 


\section{TABLE OF CONTENTS}

Page

ACKNOWLEDGMENTS

ABSTRACT $\quad$ V

LIST OF TABLES $\quad \mathrm{x}$

LIST OF FIGURES $\quad$ xi

\section{CHAPTER}

I INTRODUCTION 1

A Introduction on Efficient and Robust DSA Algorithm for SATCOM . 1

1 Dynamic Spectrum Access . . . . . . . . . . . 1

2 Types of Uncertainty in DSA . . . . . . . . . . . 2

3 Related Work - Dynamic Spectrum Access . . . . . . . . . 4

B Introduction on Graph Analysis and Embedding Algorithms . . . . . 5

1 Real-time Distributed Graph Embedding . . . . . . . . . . 6

2 Related Work - Graph Embedding . . . . . . . . . . 8

3 Hierarchical Structural Embedding of Knowledge Graph . . . 9

4 Related Work - Knowledge Graph Embedding . . . . . . . . . 12

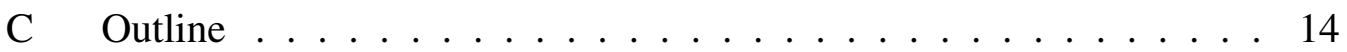

II EFFICIENT AND ROBUST DYNAMIC SPECTRUM ACCESS UNDER UNCERTAINTY (ERDSAU) ALGORITHM FOR SATCOM 16

A Problem Significance, Motivations . . . . . . . . . . . . 17

B Proposed Innovations . . . . . . . . . . . . . . . . . 22

C System Model and Mathematical Formulation For ERDSAU . . . . . 24

1 Network Model . . . . . . . . . . . . . . . 25

2 Dynamic Spectrum Access (DSA) Model . . . . . . . . . . 26 
3 Markov Process and Uncertainty . . . . . . . . . . . . 27

D LEO-Oriented Spectrum Sensing (LOSS) Algorithm . . . . . . . 30

$1 \quad$ LEO Spectrum Sensing . . . . . . . . . . . . . 31

E Key ERDSAU Notations . . . . . . . . . . . . . . . 32

III DISTRIBUTED GRAPH PARTITION AND EMBEDDING ON LARGESCALE STREAMING NETWORK 34

A Mathematical Model . . . . . . . . . . . . . . . . 34

1 Problem Formulation . . . . . . . . . . . . . 35

B Real-time Distributed Graph Partition and Embedding . . . . . . . 37

1 Graph Partition . . . . . . . . . . . . . . . . 37

2 Dynamic Graph Embedding . . . . . . . . . . . . . 41

3 Heuristic Global Aggregation . . . . . . . . . . . . 43

C Experiments .................... 45

$1 \quad$ System Setup . . . . . . . . . . . . . . . . 45

2 Graph Partition . . . . . . . . . . . . . . . 47

3 Dynamic Graph Embedding . . . . . . . . . . . . 48

4 Multi-label Classification . . . . . . . . . . . . . . . . . . 49

$5 \quad$ Link Prediction . . . . . . . . . . . . 55

$6 \quad$ Scalability . . . . . . . . . . . . . 56

IV REAL-TIME DEEP ANALYSIS OF TWITTER NETWORK 59

A Real-time Complex Twitter Network Extraction . . . . . . . . . . 62

1 Fundamental Graphs . . . . . . . . . . . . . 63

2 Complex Graph Generation . . . . . . . . . . . 64

B $\quad$ Results . . . . . . . . . . . . . . . . 67

$1 \quad$ System Setup . . . . . . . . . . . . . . . . 67

2 Twitter Network Analysis _. . . . . . . . . . . 68

$\mathrm{V}$ HIERARCHICAL STRUCTURE EMBEDDING OF KNOWLEDGE GRAPH 
A System Model . . . . . . . . . . . . . . . . . . . . 73

1 Scale-free Network . . . . . . . . . . . . . . . . . . 73

2 Mathematical Definitions . . . . . . . . . . . . . . . . 74

3 Structural Distance Calculation . . . . . . . . . . . . 75

4 Relation Similarity Calculation . . . . . . . . . . . . . 77

5 Structural Graph Creation _. . . . . . . . . . . . . 78

6 Knowledge Path Generation . . . . . . . . . . . . . . . 79

7 Latent Representation Learning _. . . . . . . . . . . 80

8 Complexity and optimization . . . . . . . . . . . 82

B Implementation and Experiments $\ldots \ldots \ldots \ldots$

1 Implementation . . . . . . . . . . . . . . . 84

2 Data Sets ................... . . 84

3 Entity Prediction $\ldots \ldots \ldots \ldots \ldots \ldots$

4 Multi-relations Prediction $\ldots \ldots \ldots \ldots$

$5 \quad$ Triple Detection . . . . . . . . . . . . . 88

$6 \quad$ Scalability . . . . . . . . . . . . . . . 89

VI CONCLUSION AND FUTURE WORK 91

A Conclusion ...................... 91

B Future Work . . . . . . . . . . . . . . . . . . . 93

REFERENCES 94

$\begin{array}{ll}\text { CURRICULUM VITAE } & 100\end{array}$ 


\section{LIST OF TABLES}

TABLE Page

1 ERDSAU Notations . . . . . . . . . . . . . . . . . 33

2 Data sets used for experiments in Section IV . . . . . . . . . . . 45

3 Multi-label classification results of BlogCatalog. . . . . . . . . . . 51

4 Multi-label classification results of Wikipedia. . . . . . . . . . . . 52

5 Multi-label classification results of YouTube. . . . . . . . . . . 53

6 Performance evaluation w.r.t. dimensionality. . . . . . . . . . 56

7 Performance evaluation w.r.t. dimensionality. . . . . . . . . . . . 58

8 Generated complex graphs . . . . . . . . . . . . . 68

9 Closest Twitter users of realDonaldTrump . . . . . . . . . . . . 70

10 Closest nodes detection . . . . . . . . . . . . . . . 71

11 Compositional Representation Operator . . . . . . . . . . 83

12 Data Sets . . . . . . . . . . . . . . . . . . 84

13 Entity Prediction. . . . . . . . . . . . . . . 85

14 Entity Prediction by Relation Types on FB38k . . . . . . . . . . . . . . . 86

15 Hits@10(\%) on FB38k ... . . . . . . . . . . . . . . 87

16 Multi-relation Prediction. . . . . . . . . . . . . . 88

17 Triple Classification . . . . . . . . . . . . . . . . . 89 


\section{LIST OF FIGURES}

FIGURE $\quad$ Page

1 Cognitive Satellite System Model . . . . . . . . . . . . . . 2

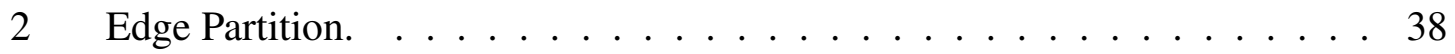

3 Subgraph Size Stand Deviation Evaluation. . . . . . . . . . . . 46

4 Maximum Subgraph Size. . . . . . . . . . . . . . . . . . . 46

5 Original Les Miserables network at (a) $T=t_{i}$, (b) $T=t_{j}$, (c) $T=t_{k}$. . . 47

6 Dynamic graph embedding results. . . . . . . . . . . . 50

7 Link prediction results using weighted-L1 operator. . . . . . . . . . . 54

8 Link prediction results using weighted-L1 operator. . . . . . . . . . . . . 54

9 Micro-F1 Performance evaluation w.r.t. \# computing nodes. . . . . . . . . 56

10 Macro-F1 Performance evaluation w.r.t. \# computing nodes. . . . . . . . . 57

11 Speed up w.r.t Number of Partitions _ . . . . . . . . . . . . 58

12 An example of the complex graph generation. . . . . . . . . . . . 66

13 Distance between realDonaldTrump and his friends under policy*), policy $y_{2}^{*}$ and policy $y_{3}^{*}$, respectively. . . . . . . . . . . . . . . 69

14 The distribution of entities appearing in random walks in FB15k follows a similar power-law distribution as in Blog data social network. . . . . . . . . 73

15 Knowledge Graph. . . . . . . . . . . . . . . . . . 75

16 Construction of Hierarchy Graph $M \ldots \ldots$. . . . . . . . . . . 78

17 Scalability of Execution Time . . . . . . . . . . . . . 90 


\section{CHAPTER I}

\section{INTRODUCTION}

A Introduction on Efficient and Robust DSA Algorithm for SATCOM

1 Dynamic Spectrum Access

Due to the spectrum scarcity, Dynamic Spectrum Access (DSA) becomes a desirable technology to improve the utilization of electromagnetic spectrum for a SATCOM system. It provides the capability that allows a Low Earth Orbit (LEO) in a SATCOM system to dynamically use free channels that are not occupied by Geosynchronous (GEO) satellites to augment the channel utilization. DSA has been extensively investigated in the last few years [1]-[2] for CR (Cognitive Radio) networks. However, most of the DSA approaches are developed for terrestrial communications without addressing the unique challenges in a SATCOM environment and these challenges include the error-prone spectrum sensing, the high mobility, the large GEO and LEO coverage, and a long signal delay due to long distance signal propagation. Fig. 1 illustrates a SATCOM system where a LEO moves in and exits the GEO coverage subsequently. The high LEO movement (i.e., about 17,000 $\mathrm{MPH}$ ) degrades the reliability of the spectrum detection. When a LEO is moving close to the edge of the GEO primary beam, its spectrum sensing results are not reliable and change drastically in a very short time. The error probability of the spectrum sensing varies with the GEO and LEO relative locations as well as the LEO mobility. In addition, 


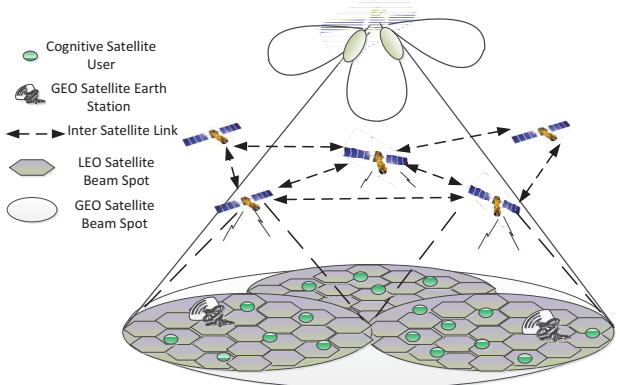

Figure 1: Cognitive Satellite System Model

GEOs and LEOs are operated in a hostile environment which is subjected to the adversarial interference. Strong interference could result in a high misdetection probability. However, most of the current DSA approaches, e.g., [3]-[4], are mainly developed for a relative static environment where the secondary users are fixed or moving with a low speed in a small geographic area, compared to the LEO's mobility. On the other hand, the LEO spectrum sensing suffers from weak GEO signals and long delay due to a long distance of the GEO signal propagation.

Decision-making is a problem to determine which channels to sense and which channels to access in a uncertainty environment. Specifically, the decision making for a cognitive SATCOM should be conducted under three types of uncertainty.

\section{Types of Uncertainty in DSA}

- Uncertainty of sensing channels: A LEO is unable to detect all spectrum channels. In other words, it only detects at most $L$ out of $N$ channels $(L<N)$ for a given time instant. A sensing strategy is needed to decide which $L$ channels to sense to achieve a high probability of finding the GEO idle channels;

- Uncertainty of sensed status: The spectrum status may not be accurately sensed by a 
LEO and the sensing results may have a high false alarm probability and a large miss detection probability. For example, the probability of false alarm could be very high (e.g., $30 \%$ or more) when external interference occurs;

- Uncertainty of spectrum access: Upon the high false alarm probability, it is a problem to determine the optimal channel for LEO to access which achieves a high data delivery ratio without collisions with the primary users.

With the consideration of the uncertainty in a SATCOM environment, this dissertation proposal presents an approach to optimize the spectrum sensing and decision making strategies for the purpose of improving the SATCOM spectrum utilization. Specifically, we formulate the spectrum sensing and access under the uncertainty as a problem of Partially Observable Markov Decision Process (POMDP). In the POMDP problem, the partial observation indicates that a secondary user, e.g., LEO, is only able to partially observe the underlying states of the primary channels. Meanwhile, the sensing errors could occur in the partially observed spectrum band. Our approach in this paper has the following contributions:

- GEO-oriented Spectrum Sensing: The spectrum sensing approach is studied to improve the LEO sensing capability to detect the GEO spectrum holes. Our sensing approach uses GEO signal-oriented cyclostationary feature detector to achieve a higher detection probability.

- POMDP Model for SATCOM: POMDP is adapted to a SATCOM system for the optimal sensing strategy, the sensor operating policy, and the access strategy by a 
two-state channel model, i.e., busy or idle, and these strategies are optimized to achieve the maximal channel utilization.

- Improved Performance: Simulations are conducted in a SATCOM environment to test the efficiency of the proposed approach. Our results include the detection probability vs. false alarm probability under different SNR environments. The accumulated throughput of single or multiple SATCOM channels are presented and our results show the improvement of the channel utilization.

\section{Related Work - Dynamic Spectrum Access}

As we mentioned above, many of the current existing DSA research works rely on the perfect sensing results for channel access and the above uncertainties are not well investigated for SATCOM data communications. Sahai et. al. [5] study the imperfect sensing. However, the sensing errors are not been considered into channel access in an optimal way. POMDP have been studied by Chen et al. [6] and their works shows that POMDP can improve the channel access efficiency under partial observation and imperfection awareness of channel. However, authors do not address the uncertainty caused by high mobility large delay in the SATCOM scenario. Furthermore, there are some DSA works [7] use the conservative access strategy to minimize the interference to the primary user. In addition, Yilmaz et al. [8] consider the problem of joint spectrum sensing and channel estimation and Liu et al. [9] present a DSA design of multiple secondary users. However, these approaches can not perform well for cognitive SATCOMs since the DSA for SATCOM under uncertainty is not well investigated which involves spectrum sensing as well as decision making for spectrum access. 


\section{B Introduction on Graph Analysis and Embedding Algorithms}

Graph Analysis draws a lot of research intentions over decades due to the omnipresence of graphs (also known as networks) in the real-world. The information of relationships/intereactions among individual units in a system can be naturally described by graph. For instance, social media network usually represents the friendship relations among user accounts, Protein-Protein interaction network can denote biology information and word co-occurrence network symbolizes linguistics models. Additionally, graphs are widely used in modern enterprise data comprised of products, orders, and transactions, which are typically recognized in form of traditional data systems [10]. Big companies are eager to have the ability of network-wide knowledge discovering of activities and relationships among users for further decision-making such as recommendation and prediction.

Previous graph analysis focused on studying of static tasks, for instance, maximum network flow and graph coloring by using classic graph theory. Also, the representation of graph was conducted by matrix, leading to a very high computational cost. In order to avoid complex matrix operations, dimensionality reduction approaches, such as principle component analysis (PDA) and multidimensional scaling (MDS), are the most frequently adopted method to graph analysis area. Modern graph analysis is much more involving with machine learning and deep learning techniques. The tasks of modern graph analysis can be categorized broadly in four applications: (1) node classification, (2) link prediction, (3) clustering, and (4) visualization [11]. However, the traditional approaches are having troubles to achieve the demanded performances. A new method named graph embedding has been proposed recently which aims to embed all the vertices of a graph into a lower 
dimensionality vector space with all the features of the graph and the relationships among the vertices are optimally encoded in the vectors. Compared to the classic graph analysis methods, graph embedding outperforms effectively and efficiently both on preserving the original similarities of the graph and modern large-scale graph processing tasks.

Embedding algorithms benefit the modern graph data analysis tasks by extracting the implicit structural information and capturing the hidden variation from high dimensional data features without complex computation. G. Hinton et al. have proposed first embedding work $[12,13]$ in hands-written recognition task by using vectors in low dimensional space to represent the high dimension of pixel intensities. Similar idea is adopted to nature language processing (NLP) area. To provide the ability of learning/reading articles to machine, the essential step is to translate the words into digital inputs. Mikolov et al. [14, 15] propose word2vec which successively represent words by encoding their semantics into $\mathrm{N}$-dimensional vectors. Due to the outstanding performance of word2vec, scientists in NLP are able to build word vector library for 13 billion words. In addition, many hard NLP tasks, for instance, machine translation, semantic analysis and question answering, are having significant process. DeepWalk [16] is the first work that applied skip-gram to social media network graph and represents the structural information of unweighted graph as vertex sequences which is generated by random walks on the graph.

\section{Real-time Distributed Graph Embedding}

While graph embedding is an intriguing idea, the existing algorithms have two major limitations: (1) None of them can perform real-time graph embedding of streaming 
data. Specifically, current graph embedding algorithms rely on prior knowledge of the entire graph and can only process the data in a batched fashion, which is not applicable to real-time streaming applications. (2) Most graph embedding algorithms are centralized, which is unable to handle big data. For example, big social networks such as Twitter and Facebook generate massive graph data (e.g., interactions) in a very short period of time. In these cases, even a super computer could quickly deplete its resources (i.e., computation, memory and storage). In fact, our own experiences have shown that "out-of-memory" is the most common error even when the data size is moderate. To overcome these limitations, it is necessary to resort to distributed graph process. While some distributed graph process frameworks (e.g., MapReduce [17], Pregel [18] and Apache Giraph [19]) have been proposed and used for iterative graph algorithms with static graph structure, none of them can handle real-time data streaming applications.

In my research, I design a streaming distributed graph processing platform which is able to distributively divide the large-scale graph data and perform all the graph embedding processes in one pass. A real-time distributed graph partition and embedding (RTDGE) algorithm also has been proposed and completed, which consists of three major steps: (i) graph partition, (ii) dynamic graph embedding, and (iii) graph aggregation. The input graph can be either real-time streaming data or batched offline data.

Data partition is an important concept in distributed big data processing. Most common method of graph partition is vertex partition which distributes vertices into un-overlapped subgraphs [20-22]. However, it is unable to guarantee an balanced graph partition due to the uncertainty of the assigned degrees of each vertex even the size of vertices assigned to each subgraph is approximately equivalent. My work investigates a 
different approach which divides the edges into distinct subsets, while vertices are associated to edges and thus may belong to several partitions. In order to accomplish the goal of processing streaming data, an adaptive negative sampling method also has been proposed which is capable of updating embedded vector by passing the training data one single time.

\section{Related Work - Graph Embedding}

The classic approach to learn the graph representations is matrix factorization technique [20, 23, 24]. Such method is designed to use the statistic information, i.e., global co-occurrence counts of the graph affinity matrix. Therefore, one major shortcoming of matrix factorization approaches is they are only considering the direct connections which is also known as first-order proximity. Additionally, matrix factorization cannot be applied to direct graphs. Usually, such approaches require the eigen-decomposition of a data matrix which is a big drawback of the computational performance.

DeepWalk [16] is the first work that adopts skip-gram from word2vec to social media network graph and represents the structural information of unweighted graph as vertex sequences which is generated by random walks on the graph. A. Grover et al. extends the graph embedding algorithm on his node2vec [25] which carefully designs the selection of the vertex sequences in order to preserve better structural information of the original graph. Both DeepWalk and node2vec first select one vertex $v_{1}$ from the graph randomly, and then select next vertex $v_{2}$ from the neighbor set of $v_{1}$. The processes are repeated until the size of the sequence is reached a pre-set number which is known as walk 
length $\theta$. The difference between DeepWalk and node2vec is that node2vec designed a biased random walk procedure. Shaosheng Cao et al. [26] have accomplished very similar work which develops shuffle sampling method to have the vertex sequences.

LINE [27] is a distinct graph embedding work which extended the skip-gram and negative sampling (SGNS) model to social media graph from the nature language processing area. In SGNS, the negative table which contains vertex pairs created stochastically from the empirical probability of the connection between two vertices plays an important role to represent the graph structure. The objective of SGNS is to train the lower dimensional vector by maximizing the positive edge and minimizing the negative pairs. Besides, LINE also significantly improved the sampling efficiency by applying alias sampling method.

Amr Ahmed et al. [20] first time use graph partition on graph factorization. In his work, a factorization technique was proposed which relies on partitioning a graph so as to minimize the number of overlapped vertices. However, such partition cannot be guaranteed to have an balanced partition. Furthermore, it requires expensive communications during the process. And as we mentioned above, factorization technique has the quadratic computational complexity.

\section{Hierarchical Structural Embedding of Knowledge Graph}

Classic graph only contains single type relation, i.e., all the edges represent same relationship among nodes. Such graph can not satisfy the modern applications. For example, a typical recommendation system includes users and products. The edge between users denotes the relationship between users, and the edge between a user and a 
product is the rating score from the user to the product. Knowledge graphs (KGs) model knowledge/fact information in the form of entities and relations. A number of KGs, such as Freebase [28], WordNet [29], DBpedia [30], YAGO [31] and NELL [32], have been created and successfully applied to many real-world applications, such as information extraction [33] [34] and question answering [35] [36]. Usually, an edge in a KG is represented as a triplet: (head entity, relation, tail entity), denoted by $(h, r, t)$, such as (Obama, BornIn, USA). Although effective in representing structured data, the underlying symbolic nature of such triplets makes KGs hard to manipulate [37].

The idea of knowledge graph embedding is to learn representations in a lower vector space of both the entities and relations meanwhile preserving their maximal relationships in the given KG. This kind of relational knowledge representation has been proved by a lot of research works [38], [39], [40], [41], [42], [43], [44] that have a better performance of facilitating various kinds of tasks, such as relation extraction, entity classification and entity prediction.

The key stone of knowledge graph embedding technique is using the representations of entity and relation to most reasonably describe the facts. The early work of knowledge graph embedding, i.e., TransE [38], is based on a translate model that assumes an equation of the representations $\vec{h}+\vec{r} \approx \vec{t}$ holds for triplet $(h, r, t)$. Despite the simplicity and efficiency of TransE model, difficulties are arisen when there are multiple relations between a pair of entities, e.g., (Obama, PresidentOf, USA) and (Obama, BornIn, USA), since only one legal $r$ is allowed by the equation. Some new translate-based algorithms such as TransR [39] and TransH [41], are proposed to tackle the disadvantage of TransE by allowing entity to have distinct representations when involved in different 
relations. But these TransE-based methods do not consider any structural information of the knowledge graph which contains rich semantic cues of the facts. Such semantic information which always conducted by relation paths, i.e., multi-hop relationships between entities, is also helpful to distinguish the multi-relations between pair-wise entities. The key challenge is how to represent the relation paths in the same vector space along with entities and relations. Because the semantic meaning of a path depends on all its constituent relations, it is reasonable to construct the path as a composition of the representations of these relations. Lin et at. [40] extend the TransE model by using three typical compositions to model the relation paths: addition, multiplication and recurrent neural network (RNN) [45]. Guu et al. [43] have proposed a similar framework, the idea of which is to build triplets using entity pairs connected not only with relations but also with relation paths. While incorporating relation paths improves model performances, the complexity of selections of relation paths is a critical challenge. Meanwhile those knowledge graph embedding approaches is limited to capture rich interactions in relational data, since the structural similarities of unlinked entities are difficult to be preserved by such relation paths. Structural embedding of knowledge graph (SE) [46] establishes an embedding from the structural information from the KGs into the lower vector space by using neural network which is an alternative method without relation path selection. More specifically, entities are represented by the lower dimensional vectors, and two separated matrices $\boldsymbol{M}_{r}^{h}$ and $\boldsymbol{M}_{r}^{t}$ to project head and tail entities respectively for each relation $r$. Then the similarity between two entities is written as: $f_{r}(h, t)=-\left|\boldsymbol{M}_{r}^{h} \cdot \vec{h}-\boldsymbol{M}_{r}^{t} \cdot \vec{t}\right|$. As a result, two entities that shared in same triplet are located closer in the embedded space. Clearly, SE only counts local structural 
relationships of entities.

I propose an original knowledge graph embedding method which embeds the entities of the knowledge graph based random walks that generated from the hierarchical context of the knowledge graph. Such hierarchy context is constructed as a multi-layer graph in which each level contains the structural similarity of entities of its corresponding multi-hop neighbors. More specifically about the hierarchical structure similarity, the bottom of the hierarchy is the degree of the entities, while at the top of the hierarchy, the similarity depends on the entire knowledge graph. Moreover, while the multi-layer graph also inflects the impact power of different relations. For instance, in the triplet (Obama, PresidentOf, USA) and (Obama, BornIn, USA), the relation PresidentOf clearly has a stronger impact on entity Obama. Besides, circular correlation composition operator is applied in the model. Hence, by using the circular correlation as the compositional operator, the proposed model is able to capture the rich interactions (multi-relation issue) but simultaneously remains efficient to computer, easy to train, and scalable to very large data sets.

\section{Related Work - Knowledge Graph Embedding}

The early work of knowledge graph embedding (Bordes et al. 2013; Socher et al. 2013;) focus on exploring different objective functions to model direct relationships between two entities, such as the TransE-based Methods (e.g., TransE [38], TransH [41], TransR [39]). The basic idea behind all translation-based models is that the relation is regarded as a translation from head to tail when it is encoded into a metric space, that is, $\vec{h}+\vec{r} \approx \vec{t}$ holds for the triplet $(h, r, t)$. This assumption results in relation completion 
by finding an $\vec{r}^{*}$ such that it corresponds to one of the nearest neighbors of $\vec{r}$, that is, $\vec{h}+\vec{r}^{*}=\vec{t}$ for a given entity pair $(h, t)$. TransH [41] follows the general idea of translationbased model, by introducing relation-specific hyperplanes. It embeds each relation $r$ as a vector $\vec{r}$ on a hyperplane with a corresponding normal vector $\vec{w}_{r}$. Then a given triplet $(h, r, t),\left\|\vec{h}_{\perp}+\vec{r}-\vec{t}_{\perp}\right\|_{2}^{2} \approx 0$ holds, where $\vec{h}_{\perp}=\vec{h}-\vec{w}_{r}^{T} \vec{h} \vec{w}_{r}$, and $\vec{t}_{\perp}=\vec{t}-\vec{w}_{r}^{T} \vec{t} \vec{w}_{r}$ are the corresponding vectors when head $h$ and tail $t$ are projected to relation $r$ ' hyperplane. TransR [39] shares a very similar model as TransH, but only it propose a relation-specific space instead of the hyperplane. A translation matrix $M_{r} \in \mathbb{R}^{k x d}$ is introduced to project the entity space to the relation space of relation $r$. Hence, the corresponding vectors in the relation-specific space of $r$ is given as: $\vec{h}_{\perp}=M_{r} \vec{h}$ and $\vec{t}_{\perp}=M_{r} \vec{t}$. CTransR is developed as an extension of TransR, which clusters diverse head-tail entity pairs into groups and learns distinct relation vectors for each group.

Several recent approaches (Guu et al. [43]; Toutanova et al. [47]; Lin et al. [40]) demonstrate limitations of prior approaches relying upon vector-space models alone. For example, when dealing with multi-step (compositional) relationships (e.g., (Obama, BornIn, Hawaii), (Hawaii, PartOf, USA)), direct relationship-models suffer from cascading errors when recursively applied their answer to the next input. Hence, recent works propose different approaches of injecting multi-step relation paths from observed triplets during training, which further improve performance in knowledge graph tasks. For instance, Lin et al., [40] and Gu et al., [43] also encode multiple-step relation path information into $\mathrm{KG}$ representation learning. In [43], for a given pair of entities $(h, t)$, if there is path $p: r_{1} \rightarrow r_{2} \rightarrow \ldots \rightarrow r_{l}$ can be found between them, a new triplet is constructed as $(h, p, t)$. To model this path-connect triplets. Guu et al. extend TransE as 
$-\left\|\vec{h}+\left(\vec{r}_{1}+\ldots+\vec{r}_{l}+\vec{t}\right)\right\|$. It also extends another model [48] to $\vec{h}_{\perp}\left(\boldsymbol{M}_{1} \circ \ldots \boldsymbol{M}_{l}\right) \vec{t}_{\perp}$.

Although it achieves better performance, using multi-step relation paths also introduces some technical challenges. Since the number of possible paths grows exponentially with the path length, it is prohibitive to consider all possible paths during the training time for knowledge bases such as FB15K. Existing approaches need to complexly designed procedures for sampling or pruning paths of observed triplets in the symbolic space. As most paths are not informative for inferring missing relations, these approaches might be suboptimal.

Hoffmann et al. [49] propose a weak supervision information extraction algorithm which is capable of modeling overlapping relations. But it focuses on extracting the facts of entities from natural language sentences and is only able to learn the sentence-level embedding presentations. The future similar works, such as SE [46], also concentrate on finding/reconstructing the mission data(i.e., missing entities or relations) from random web data which fails to dig structural information of the original network.

\section{Outline}

This dissertation is organized as follow. In the next chapter (Chapter II), the SATCOM network model in the uncertain wireless communication environment is described. Besides, the optimized spectrum sensing and POMDP-based decision making model are also represented in Chapter II. Then, the distributed real-time graph embedding approach is demonstrated in Chapter III including the detailed system model and experimental results. A real-world application: Twitter network analysis has been introduced in Chapter III which is implemented on the proposed platform. Hierarchical 
Structural Embedding of Knowledge Graph (HSE) is introduced in Chapter V. The last chapter is the conclusion and future work. 


\section{CHAPTER II}

\section{EFFICIENT AND ROBUST DYNAMIC SPECTRUM ACCESS UNDER UNCERTAINTY (ERDSAU) ALGORITHM FOR SATCOM}

Dynamic spectrum access (DSA) has been extensively investigated over the past few years under the name of cognitive radio. Using DSA, the secondary users can utilize licensed spectrums to transmit their data without affecting the primary users. Figure 1 illustrates a cognitive satellite communication (SATCOM) system where the Low Earth Orbit (LEO) satellites dynamically utilize the unused spectrum of Geosynchronous (GEO) satellites. GEO satellite can reduce its communication payload by sharing tasks and spectrum reuse with LEO satellites. For such a purpose, LEO performs spectrum sensing and makes decision on spectrum access. The spectrum sensing can be conducted with or without the cooperation among LEO satellites, depending on the inter-satellite links. Aggregation and fusion again enhance the accuracy on the decision making. It is because each LEO satellite is mostly limited in its capabilities of sensing all spectrum bands and knows their status. Figure 1 also shows that GEO and LEO satellites beam spots are well formulated in a hierarchical way. A number of LEO hexagonal beam cells are located with the coverage of GEO to achieve a full coverage on the ground. The example in Figure 1 only shows one spot beam of GEO satellite downlink transmission and it can be generalized to the whole SATCOM system. 


\section{A Problem Significance, Motivations}

Due to the spectrum scarcity, Dynamic Spectrum Access (DSA) becomes a desirable technology over the past few years under the name of cognitive radio to improve the utilization of electromagnetic spectrum, especially for a satellite communication (SATCOM) system. Using DSA, the secondary users can utilize the licensed spectrums to transmit their data without affecting the primary users. Hence, a Low Earth Orbit (LEO) in a SATCOM system can be provided the capability to dynamically access free channels that are not occupied by Geosynchronous (GEO) satellites to augment the channel utilization. DSA has been extensively investigated in the last few years [1]-[2] for CR (Cognitive Radio) networks. However, most of the DSA approaches are developed for terrestrial communications without addressing the following unique challenges in a SATCOM environment:

- Error-prone Spectrum Sensing: Spectrum sensing in SATCOM environments is much more difficult compared to the terrestrial environments due to the long distance of the satellite transmissions. In this case, the sensor usually receives weak signals with long propagation delay, making accurate and timely detection a challenge. The LEO spectrum sensing may deviate from true of the spectrum status. In addition, a LEO can only sense a partial GEO spectrum bands.

- High Mobility: The high moving speed of LEO satellites (i.e., about 17,000 MPH) affects the reliability of the spectrum detection. With high mobility, LEO satellites need to constantly sense the spectrum and update the sensing results. For those LEO satellites near the edge of the primary beam, the sensing results can change drastically 
in a short time. The error probability of the spectrum sensing varies with the LEO locations and LEO mobility.

- Larger Coverage: The LEO satellites are usually separated far away from each other. This creates significant transmission delay when sensing data are exchanged among the sensors or collected at the coordinator that performs data aggregation and fusion. The transmission delays accordingly results in the delay on decision making while the spectrum status may change. Furthermore, due to the large area deployment of the sensors, integrating of sensing results from multiple sensors is difficult and may lead to a wrong decision at a specific location.

- High Interference or Jamming: For practical applications, GEOs and LEOs are operated in a high interference even hostile jamming environment. Those high or jamming interference could result in high high midsection probability (i.e., false alarm probability). Moreover, current DSA algorithms are unable to address the spectrum access when jamming occurs.

In this work, we leverage the existing sensors on LEO satellites using energy detection for cooperative spectrum sensing. A LEO satellite detects GEO downlink transmission by using directional antennas pointing to the GEO satellite. For the GEO earth station uplink transmission, each LEO satellites shall detect GEO ground users from all directions and make cooperative decisions about GEO spectrum availability. Based on such a model, our goal is to devise the algorithms to address DSA decision making under three types of uncertainties:

- Uncertainty of sensing channels: A LEO is unable to detect all spectrum channels. 
In other words, it only detects at most $L$ out of $N$ channels $(L<N)$ for a given time instant. A sensing strategy is needed to decide which $L$ channels to sense to achieve a high probability of finding the GEO idle channels;

- Uncertainty of sensed status: The spectrum status may not be accurately sensed by a LEO and the sensing results may have a high false alarm probability and a large miss detection probability. For example, the probability of false alarm could be very high (e.g., $30 \%$ or more) when external interference occurs;

- Uncertainty of spectrum access: Upon the high false alarm probability, it is a problem to determine the optimal channel for LEO to access which achieves a high data delivery ratio without collisions with the primary users.

In order to optimize the spectrum sensing and decision making strategies for the purpose of improving the SATCOM spectrum utilization with the consideration of the uncertainty in a SATCOM environment, our work propose a novel Efficient and Robust Dynamic Spectrum Access under Uncertainty (ERDSAU) algorithms. ERDSAU algorithms address above uncertainties and aforementioned challenges to provide optimal spectrum utilization at the LEO satellites, without degrading the GEO service of quality. Specifically, it is able to filter the interference, jamming signals and intelligently recognize the GEO presence by a GEO Signal-oriented Cyclostationary Feature Detector. Furthermore, it formulates the DSA uncertainties as a problem of Partially Observable Markov Decision Process (POMDP). Partial observation indicates that a LEO satellite is only able to sense a partial of spectrum channel. The secondary users (i.e., LEO satellites) partially observe the underlying states of the primary channels and sensing errors could 
occur in the the partially observed spectrum band. Under partial observation and imperfection awareness of channel, POMDP is an optimization problem that allows a LEO satellite to optimally take action on the spectrum channel. In a collaborative way, ERDSAU tracks each spectrum channel by a probability distribution over the set of possible states that is evaluated on a set of observations and observation probabilities and the underlying Markov decision process, providing high accuracy on decision making. Figure 2 illustrates our proposed ERDSAU algorithms. In ERDSAU, each LEO satellite acts as a fusion center and makes a joint decision. The well-developed POMDP theory allows us to develop the robust algorithms for solving the spectrum accessing uncertainties such that a LEO satellite can optimally decide whether or not to transmit its data on the observed channel.

ERDSAU proposes five algorithms to fill the technical gaps which have been left open in the existing approaches:

- Cyclostationary Feature Detector with Adaptive Detection Threshold: The detection threshold is adaptive to SNR in the interfering environment. It improves the DSA sensing capabilities by achieving much better performance in terms of Probability of Detection and Receiver Operating Characteristics, compared to Energy detector and Matched Filter Detector. Besides, adaptive detection threshold improves performance, compared to traditional Cyclostationary Feature Detector that employs constant detection threshold.

- LEO-Oriented Spectrum Sensing (LOSS) Algorithm: LOSS designs smart antenna array by considering Beanforming and DoA to differentiate the GEO and non-GEO signals. LOSS successively improves the spectrum sensing accuracy by 
reaching almost $100 \%$ of detection probability when the probability of false alarm is even less than $5 \%$. Furthermore, LOSS is able to mitigate the detection delay.

- Single LEO Satellite DSA (SLSD) Algorithm: SLSD is proposed for the case of a single satellite. It presents a mathematical constrained POMDP model for decision making to provide accurate decision making for LEO spectrum sensing. SLSD develops the separation principle to decouple the POMDP optimization constrains. SLSD allows optimization of the spectrum sensing and access strategies.

- Multiple LEO Satellite DSA (MLSD) Algorithm: MLSD algorithm is developed to integrate the sensing results from multiple sensors specially tailored for the SATCOM environments. Due to the hardware limitations, each LEO satellite can only sense a relatively small portion of the entire frequency band. Therefore, the sensed information is far from being sufficient for precisely determining the wide range of unused channels. MLSD addresses this issue by utilizing the sensed information from several LEO satellites. Particularly, SLSD algorithm collects the local sensing results, and then these results are weighted by the fusion center for final decision making. MLSD implements different fusion rules and considers the geographical information to improve the fusing accuracy. MLSD is the first approach that uses geographical information for spectrum sensing.

- Optimizing Joint Design of DSA (OJDD) Algorithm: The SLSD and MLSD decision making is conducted under imperfect awareness. We further incorporate the practical concerns into our decision making process to avoid potential interference with the GEO transmissions while maintaining the robustness of the 
cognitive LEO systems. OJDD prioritizes the LEO satellites in detecting spectrum holes to improve the resource allocation among multiple satellites. This approach allows the time-sensitive LEO satellites data to be delivered on time thus improves the performance.

\section{B Proposed Innovations}

ERDSAU models the spectrum access as a POMDP optimization problem that maximizes LEO satellites capacity under the GEO satellite communications collision provisions. It therefore implements a dynamic fusion process that improves the LEO systems performance under the primary GEO systems collision constraint and delay condition. The unique challenge on the satellite environments are well addressed. Our theoretical analysis indicates the following technical benefits for co-existing GEO and LEO environment:

- Improve Spectrum Sensing Accuracy: Cyclostationary Feature Detector and LEO-Oriented Spectrum Sensing (LOSS) Algorithm are developed to increase the accuracy of dynamic spectrum sensing, evaluated by detection probability and false alarm probability. Cyclostationary Feature Detector achieves the higher accuracy without needing any sophisticate sensors. Besides, ERDSAU takes advantage of POMDP to ensure the detection precision during the spectrum sensing and access. POMDP is well applicable for partial observed decision-making problem.

- Rapid Response: ERDSAU considers the unique LEO geographical information on the data fusion. The fusion rules are well design such that it can fast response to the status change on the spectrum channels. It also has the ability to optimally select the 
satellites for cooperation once the geographical information cannot precisely guide the cooperation. It updates the spectrum status with the ever fast changing sensing environment

- Computation Efficiency: ERDSAU presents a jointly design the decision making algorithm while decoupling the cooperative sensing on optimization. It achieves the high efficiency on data fusion with very little communication requirements among satellites.

- Optimize Spectrum Accessing Capability: The other three algorithms are designed to optimize the spectrum accessing for maximal spectrum utilization while restricting the collision probability in a GEO satisfiable threshold. In essential, ERDSAU models the DSA in the SATCOM environment as a POMDP problem. Partial observation indicates that a LEO satellite is only able to sense a partial of spectrum channels, e.g., 5 out of 15 GEO channels. Under partial observation and imperfection awareness of channel, POMDP is an optimization problem that allows a LEO to optimally take actions on the spectrum channel. Three algorithms are:

- SLSD Algorithm: Given a dynamic sensing environment with inevitable sensing errors and imperfectness, SLSD algorithm provide an efficient and optimal scheme using which the LEO satellite can decide the sensing and access actions such that it maximizes the transmission rate in the expectation sense. The single satellite algorithm uses the separation principle to highly reduce the sensing and processing complexity. This algorithm is further developed in the case where multiple LEO satellites are connected for 
performance improvement.

- MLSD Algorithm: In the case of multiple LEO satellites, MLSD algorithm is devised to integrate the sensed data to improve the sensing accuracy. MLSD proposes three different data fusion rules depending on the available information: (i) each satellite shares the final local sensing decision to the fusion center; (ii) each satellite shares the local sensing information instead of the final decision to the fusion center; (iii) sequential cooperative decision making with known geographical information. The cooperative sensing can significantly improve the accuracy of the sensing results. One important factor which is incorporated in the MLSD algorithm is the geographical information. By exploiting this information, the accuracy of decision making in the SATCOM environments can be substantially improved.

- OJDD Algorithm: The OJDD algorithm is proposed to incorporate the practical concerns into our decision making results to avoid the potential interference with GEO users while maintaining the robustness of the cognitive LEO systems. In particular, we prioritize the LEO satellites and detect spectrum holes to better allocate the resources among LEO satellites.

\section{System Model and Mathematical Formulation For ERDSAU}

This section is aiming to describe and clarify the terms and assumptions for developing ERDSAU algorithms. 


\section{Network Model}

We first describe the satellite network and communication models before discussing our proposed ERDSAU algorithms. Figure1 illustrates a cognitive satellite communication (SATCOM) system that includes Low Earth Orbit (LEO) and Geosynchronous (GEO) satellites. Figure1 shows the hierarchical SATCOM network:

- GEO and Its Links: GEO satellites are considered as the primary users in the satellite network. They are located in orbit $35,863 \mathrm{~km}$ above the earths surface along the equator, and revolve around the earth at the same speed as the earth rotates. Thus, they remain in the same position relative to the surface of earth. The GEO and the satellite earth stations, for example, can communicate by certain allocated spectrum, e.g., C band (3.4 C 6.65G Hz). In particularly, GEO is mostly used for broadcast and multipoint applications. GEO satellites distance also causes it to have both a relatively weak signal and a time delay in the signal.

- LEO and Its Links: LEO satellites are much closer to the earth than GEO satellites as they are located from 500 to $1,500 \mathrm{~km}$ above the surface. LEO satellites do not stay in fixed position relative to the surface and they are only visible for 15 to 20 minutes during a pass. Internal LEO links can be established for the communication between the LEOs, formulating a LEO network. On the other hand, LEO provides broadcast or point-to-point communications to the earth stations. As shown in Figure1, a LEO satellite has a much smaller coverage, compared to that of GEO.

- Earth Stations and Its Links: The earth stations are located on the ground and could connect to GEOs or LEOs. 
GEO, LEO, and Earth Stations form hierarchical network structure where GEO is located on the top, LEO is situated in the middle, and the Earth Stations are located on the GEO and LEO's coverage. They are interconnected (e.g., directional or bidirectional) on each other, depending on the actual applications. In the next subsection, we further discuss cognitive spectrum utilization on the satellite network.

2 Dynamic Spectrum Access (DSA) Model

The satellite frequency is divided into subcarriers (e.g., channels) and DSA allows dynamic utilization of these subcarriers in an effective way. Figure1 shows three types of satellite communicating links in the SATCOM network:

- Primary User: GEO is the primary user and let $\mathbf{N}=\{1,2, \ldots, N\}$ be the set of licensed channels that are used by GEO. FDMA, CDMA, or their variations can be used for them to share the number of $N$ channels among GEO communications.

- Seconder User: LEO is the second user as it could access $N$ channels, subject to the constraints imposed by GEO. The LEO links using these channels are cognitive links. In addition to the cognitive links by using GEO channels, LEOs could have their own licensed channels. There are two modes for the cognitive links:

- Overlay mode: LEO exploits a licensed channel only when GEO is absence of usage.

- Underlap mode: LEO can access the licensed channels even in the presence of GEO usage, however, subject to an interference constraint in a way to guarantee the GEOs performance. Suppose a pair of LEO where A is the transmitter and B 
is its intended receiver. A channel is an opportunity to A or B if these two LEOs can communicate successfully over this channel while limiting the interference to GEO below a predefined level determined by the regulatory policy, denoted by $\eta$

Our ERDSAU algorithms could work for both spectrum access modes. Its overall objective is to improve the LEO capability by using the licensed user while protecting the spectrum licenses from interference.

\section{Markov Process and Uncertainty}

The problem of decision making for SATCOM is that LEO dynamically determines the optimal licensed channels for augmenting its link capabilities, ensuring the GEOs performance. Let $B_{i}$ be the bandwidth of the $i$ th licensed channel in $N$ if it is occupied by LEO. Let $\mathbf{S}(t)=\left\{S_{1}(t), S_{2}(t), \ldots, S_{N}(t)\right\}$ represent the channel state at time slot $t$, where:

$$
s_{i}(t)=\left\{\begin{array}{rr}
0, & s_{i} \text { is busy } \\
1, & s_{i} \text { is idle }
\end{array}\right.
$$

The busy state means the $i$ th channel is occupied by GEO and otherwise it is idle. The channel states transfer over the time domain which can be stated by a discrete time Markov process which has a total of $M=2^{N}$ states. For the Markov process, let

$$
P=\operatorname{Pr}\left[s(t+1)=s^{\prime} \mid s(t)=s\right]
$$

be the transition probability. 
In the Markov process, LEO seeks for temporal spectrum holes to opportunistically access the idle channels for transmitting its data. $S_{i}(t)$ is unknown to LEO and it is responsible for tracking the channel states that are dynamic in both the time and space domain. However, SATCOM is an uncertainty environment caused by:

- Partial Sensing: A LEO can only sense a portion of licensed channels, due to timevariations in the dynamic channel range and bandwidth of the signals to be detected.

- Error-prone Spectrum Sensing: The LEO sensed $S_{i}(t)$ state may be different to the actual GEO state. As we stated in above Subsection, GEO is located far from the LEO and the GEO transmitted signals are relatively weak after long distance propagation, resulting in high error sensing probability.

- Noise and High Interference: GEO signals received at a LEO are generally contain noise, caused by interference. A low and noise signal results in a low SNR (signal to noise ratio) which again imposes the difficulty to detect the GEO signals, increasing the high error sensing probability.

- High Mobility: As we mentioned in the Introduction section, the LEO moves in a very high speed. In a high mobile environment, it is a challenge for LEO to constantly sense the spectrum and update the sensing results $S_{i}(t)$ for each channel. It is even more difficulty when the LEO is moving in or out of the GEOs coverage.

- Large Coverage: GEO has a large coverage and there is a long distance between the GEO and LEO. The long distance propagation of the GEO signals before or after LEOs sensing will affect the sensing results. 
The $S_{i}(t)$ uncertainty is modeled by three parameters:

- Set of Sensing Channels $\mathbf{L}(\mathbf{t})$ : We define $\mathbf{L}(t)=\{1,2, \ldots, L\}$ be set of the channels under the LEOs spectrum sensing at a given time $t$, where $\mathbf{L}(\mathbf{t})$ is a subset of $N$, i.e., $\mathbf{L} \subseteq \mathbf{N}$

- False Alarm Probability $\varepsilon(t)$ : Among the $\mathbf{L}(t)$ channels, the sensing results are not perfect and $\varepsilon(t)$ is the probability that channel is in the state of $s_{i}(t)=1$, i.e., idle, but the sensing result is $s_{i}(t)=0$, i.e., busy. If a channel is falsely alarmed, the opportunity of using this channel at time $t$ will be lost by LEO.

- Misdetection Probability $\delta(t)$ : Among the $\mathbf{L}(t)$ channels, $\delta(t)$ is the probability that channel is in the state of $s_{i}(t)=0$, i.e., busy, but the sensing result is $s_{i}(t)=1$, i.e., idle. The use of the channel $s_{i}(t)$ will degrade the GEOs performance, due to interference.

For simplicity of expression, we use $\pi_{s}$ to denote the sensing policy that is the set of channels planned for spectrum sensing, and $\pi_{\delta}$ to denote sensor operating policy associated by $(\varepsilon, \delta)$ probabilities, $\pi_{c}$ to represent the set of channels for transmitting data and each channel is associated with a pair of transmission probabilities:

- $f_{a}(0)$ is defined as the transmission probability when the channel state of sensing is busy, i.e., $S_{a}(t)=0$.

- $f_{a}(1)$ is defined as the transmission probability when the channel state of sensing is idle, i.e., $S_{a}(t)=1$. 
With the above definitions, the decision making at time $t$ under uncertainty for DSA is the problem that LEO:

- Determine the sensing channel set $\mathbf{L}(\mathbf{t})$ to perform sensing, i.e., policy $\pi_{s}$ is carried out,

- LEO performs sensing on the selected channels and evaluates the sensing reliability of the sensing results, denoted by $(\varepsilon, \delta)$, i.e., policy $\pi_{\delta}$ is carried out, and

- Determine the set of channels for the LEO to use and the transmission probabilities $\left\{f_{a}(0), f_{a}(1)\right\}$ for the chosen channels, i.e., $\pi_{c}$ is carried out. The determination of $\pi_{c}$ is based on the $(\varepsilon, \delta)$ policy and the constraints imposed by GEO, i.e., without affecting the GEOs performance or the interference to GEO is under predefined level (e.g., $\eta)$

In the next section, we first depict the SLSD algorithm in the scenario that each LEO independently performs decision making on the spectrum access. This algorithm is then extended to LEO collaborative decision making.

\section{LEO-Oriented Spectrum Sensing (LOSS) Algorithm}

LOSS algorithm is to optimize the spectrum sensing to achieve a high probability of GEO signal detection, i.e., $1-\delta$, where $\delta$ is the misdetection probability and a low probability of false alarm, i.e., $\varepsilon$. Currently, there are three types of spectrum sensing schemes: (i) Energy Detector, (ii) Matched Filter detector, and (iii) Cyclostationary feature detector. The evaluation of $(1-\delta, \varepsilon)$ of these three schemes in an uncertainty environment should be considered in a high interference or jamming environment. 
Therefore, LOSS algorithm should achieves excellent $(1-\delta, \varepsilon)$ in a high interference or jamming environment.

\section{LEO Spectrum Sensing}

There are two results for a LEO spectrum sensing detector for a given GEO channel: the absence of the signal or the presence of the signal, denoted by $H_{0}, H_{1}$ respectively, presented by:

$$
\begin{aligned}
& H_{0}: \quad x(t)=n(t) \\
& H_{1}: \quad x(t)=s(t) h(t)+n(t)
\end{aligned}
$$

where $x(t)$ represents the received signal by LEO detector, $s(t)$ is the original transmitted signal, $n(t)$ is noise signal and $h(t)$ is channel gain. It is noted that the jamming signal could be included in the noise or separated as we will further discuss. We first theoretically review the spectrum sensing schemes:

- Energy Detector: The energy detector compares the power of received signal against a threshold:

$$
\begin{aligned}
& H_{0}: y(k)=\sum_{\substack{k=0 \\
M-1}}^{M-1}|x(k)|^{2}<\lambda \\
& H_{0}: y(k)=\sum_{k=0}^{M}|x(k)|^{2}>\lambda
\end{aligned}
$$

where $\lambda$ is the threshold and $M$ is the number of sampling times. $H_{0}$ holds if the received power is less than the threshold. Otherwise, $H_{1}$ holds. The probability of false alarm (PFA) and detection probability (PD) can be calculated by the following equations: 


$$
\begin{gathered}
P_{D}=\operatorname{Pr}\left\{y>\lambda \mid H_{1}\right\}=1-\Gamma\left(M, \frac{\lambda}{\left(\sigma_{n}^{2}+\sigma_{s}^{2}\right)}\right) \\
P_{f}=\frac{\Gamma\left(\frac{M}{2}, \frac{\lambda}{2 \sigma_{n}^{2}}\right)}{\Gamma\left(\frac{N}{2}\right)}
\end{gathered}
$$

where $\sigma_{n}^{2}, \sigma_{s}^{2}$ are the variances of noise and original signal respectively. $\Gamma(\dot{)}$ is incomplete gamma function.

- Matched filter design: The matched filter detector is expressed as:

$$
y(n)=\sum_{k=0}^{M-1} x(n) x_{r}(n-k)
$$

where, $x(n)$ is the input transmitted signal, $x_{r}(t)$ is the stored GEO's signal, $y(n)$ is the matched filter output, $n$ represents the sampling sequence ( $M$ times sampling), $k$ is the coefficient of filter. Let $\lambda$ be the threshold and $y(n)>\lambda$ means the GEO signal is present. Otherwise, the channel will be idle. The probability of false alarm of matched filter detection is:

$$
P_{f}=\exp \frac{-\lambda_{2}}{E \sigma^{2}}
$$

where $E$ is the input signal power and $\sigma^{2}$ is the average white Gaussian noise variance. The probability of detection is:

$$
P_{D}=Q\left(\sqrt{\frac{2 E}{\sigma^{2}}}, \sqrt{\frac{2 \lambda^{2}}{E \sigma^{2}}}\right)
$$

where $Q(\dot{)}$ is Generalized Marcum Q-function.

\section{E Key ERDSAU Notations}


TABLE 1

ERDSAU Notations

\begin{tabular}{|c|c|c|c|}
\hline Symbols & Description & Symbols & Description \\
\hline $\begin{array}{l}\mathbf{N}= \\
\{1,2, \ldots, N\end{array}$ & Set of GEO Channels & $t$ & Time slot \\
\hline$x_{G}(t)$ & GEO Signal & $x_{J}(t)$ & Jamming Signal \\
\hline$n(t)$ & White Gaussian Noise & $\mathbf{Y}(t)$ & $\begin{array}{l}\text { Combined effective signal } \\
\text { of GEO, Jamming, and } \\
\text { noise signals. }\end{array}$ \\
\hline $\mathbf{y}(t)$ & $\begin{array}{l}\text { Received signal at LEO } \\
\text { antenna array }\end{array}$ & $\mathbf{R}_{y y}$ & $\begin{array}{l}\text { Covariance matrix of array } \\
\text { output signal }\end{array}$ \\
\hline$\lambda$ & $\begin{array}{l}\text { Eigenvector of covariance } \\
\text { matrix }\end{array}$ & $\hat{x}_{G}(t)$ & $\begin{array}{l}\text { GEO signal after filtering } \\
\text { the jamming and noise } \\
\text { signals }\end{array}$ \\
\hline $\mathbf{S}(t)$ & $\begin{array}{l}\text { Set of GEO channel state by } \\
\left\{S_{1}(t), S_{2}(t), \ldots, S_{N}(t)\right\}\end{array}$ & $S_{i}(t)$ & $\begin{array}{l}\text { The state of channel } i \text { at time } \\
\text { slot } t \text { (" } 0 \text { " for busy and " } 1 \text { " } \\
\text { for idle) }\end{array}$ \\
\hline $\begin{array}{l}\mathbf{L}(t) \quad= \\
\{1,2, \ldots, L\}\end{array}$ & $\begin{array}{l}\text { Set of sensing channels by a } \\
\text { LEO }\end{array}$ & $M=2^{N}$ & $\begin{array}{l}\text { Total number of channel } \\
\text { states }\end{array}$ \\
\hline$\varepsilon, P_{f}(t)$ & $\begin{array}{l}\text { False alarm probability on } \\
\text { sensing }\end{array}$ & $\delta$ & Misdetection probability \\
\hline$\pi_{\delta}$ & Sensor operating policy & $\pi_{s}$ & Sensing policy \\
\hline$\pi_{c}$ & Access policy & $\Theta_{a}(t)$ & $\begin{array}{l}\text { Sensing result of channel } a \\
\text { in slot } t\end{array}$ \\
\hline$\Phi_{a}(t)$ & $\begin{array}{l}\text { Access action of channel } a \\
\text { in slot } t\end{array}$ & $R(t)$ & $\begin{array}{l}\text { Reward which is the } \\
\text { throughput gain by using a } \\
\text { channel in time slot } t\end{array}$ \\
\hline $\mathbf{A}_{a}(t)$ & $\begin{array}{l}\text { Sensing action space: a set } \\
\text { of channels could be sensed } \\
\text { in slot } t\end{array}$ & $\mathbf{A}_{\delta}(t)$ & $\begin{array}{l}\text { Sensor operating space: a } \\
\text { feasible region of sensor } \\
\text { operating parameters of } \\
\text { channel } a\end{array}$ \\
\hline$\overline{\mathbf{\Lambda}(t)}$ & Belief vector & $A c k_{a}(t)$ & $\begin{array}{l}\text { Acknowledgement } \\
\text { channel } a \text { in slot } t\end{array}$ \\
\hline$f(0)$ & $\begin{array}{l}\text { Transmission probability } \\
\text { when sensing result is " } 0 \text { " } \\
\text { (busy) }\end{array}$ & $f(1)$ & $\begin{array}{l}\text { Transmission probability } \\
\text { when sensing result is " } 1 \text { " } \\
\text { (idle) }\end{array}$ \\
\hline$\lambda_{s}(t)$ & $\begin{array}{l}\text { Conditional probability that } \\
\text { the spectrum occupancy } \\
\text { state is s at slot } t\end{array}$ & $P C_{a}(t)$ & Collision probability \\
\hline$B_{i}$ & The bandwidth of channel $i$ & $\omega_{i}(t)$ & $\begin{array}{l}\text { The probability that channel } \\
i \text { is available in time slot } t\end{array}$ \\
\hline$\alpha_{i}(t)$ & $\begin{array}{l}\text { The transition probability } \\
\text { from state " } 0 \text { " to state " } 1 \text { " }\end{array}$ & $\beta_{i}(t)$ & $\begin{array}{l}\text { The transition probability } \\
\text { that channel } i \text { stays in state } \\
\text { " } 1 \text { " }\end{array}$ \\
\hline$\zeta$ & $\begin{array}{l}\text { Maximum collision } \\
\text { probability where the } \\
\text { case that a LEO seizes a } \\
\text { channel that GEO is using is } \\
\text { considered as a collision }\end{array}$ & & \\
\hline
\end{tabular}




\section{CHAPTER III}

\section{DISTRIBUTED GRAPH PARTITION AND EMBEDDING ON LARGE-SCALE STREAMING NETWORK}

This chapter presents a new graph embedding framework named as real-time and distributed graph embedding (RTDGE), which can distributively embed large scale graph in real-time. Specifically, we proposed an edge based graph partition to ensure balanced partition. To handle streaming data input, a dynamic graph embedding approach was provided without compromising the system efficiency and effectiveness. Then, we adopted a heuristic global aggregation method to combine the locally embedded vector spaces. Finally, our RTDGE algorithm was implemented and evaluated on the planform which combined with Apache Kafka, Apache Zookeeper and Apache Storm. The experimental results on various real-world data sets prove the effectiveness of our algorithm.

\section{A Mathematical Model}

Let $G(V, E)$ be a large graph, where $V$ and $E$ are respectively the vertex set and edge set with $|V|=N$. Edge $e_{i j}=\left(v_{i}, v_{j}\right)$ is defined as the directed link from vertex $v_{i}$ to $v_{j}$ with associated weight $\omega_{i j}$. The goal of graph embedding is to map the original graph to a $d$-dimensional feature representation vector space $(d<<N)$ while the original 
similarities among vertices are maximally preserved. Accordingly, the optimization of graph embedding can be mathematically written as:

$$
O=\min \left(s\left(\vec{v}_{i}, \vec{v}_{j}\right)-s\left(v_{i}, v_{j}\right)\right),
$$

where $\vec{v}_{i}$ and $\vec{v}_{j}$ are the embedded vectors for vertex $v_{i}$ and $v_{j}$, and $s(\cdot)$ is a pre-defined similarity function.

In this paper, we adopt the similarity defined in LINE [27] and extend that to the scenario of dynamic and distributed graph embedding. Specifically, the similarity is defined in two aspects: (i) first-order proximity, and (ii) second-order proximity. The first-order proximity is defined as the direct connection between two vertices. Since the first-order proximity is insufficient to present the global structure of the graph, we also use the secondorder proximity, which is defined as the number of shared neighbors between two vertices. For the graph shown in Fig.2, using the first-order proximity, the embedded vector $\vec{v}_{1}$ should be closer to $\vec{v}_{2}$ than to $\vec{v}_{7}$ since vertices $v_{1}$ and $v_{2}$ are directly connected in the original graph. Using the second-order proximity, $\vec{v}_{6}$ should be closer to $\vec{v}_{1}$ than to $\vec{v}_{5}$ because $v_{6}$ and $v_{1}$ have more shared neighbors in the original graph.

\section{Problem Formulation}

According to LINE [27], for the first-order proximity, the similarity between vertices $v_{i}$ and $v_{j}$ (i.e., strength of their direct connection) is calculated as the following empirical probability:

$$
s\left(v_{i}, v_{j}\right)=p_{1}\left(v_{i}, v_{j}\right)=\frac{\omega_{i j}}{W},
$$


where $W=\sum_{e \in E} \omega_{e}$ is the total weights.

After graph embedding, the similarity between embedded vectors $\vec{v}_{i}$ and $\vec{v}_{j}$ is calculated as the following probability:

$$
s\left(\vec{v}_{i}, \vec{v}_{j}\right)=\hat{p}_{1}\left(\vec{v}_{i}, \vec{v}_{j}\right)=\frac{1}{1+\exp \left(-\vec{v}_{i}^{T} \cdot \vec{v}_{j}\right)} .
$$

Let $d(\cdot)$ denote the KL distance, and the optimization problem Eq.(10) becomes:

$$
O_{1}=\min d\left(\hat{p}_{1}\left(\vec{v}_{i}, \vec{v}_{j}\right), p_{1}\left(v_{i}, v_{j}\right)\right)
$$

where vectors $\vec{v}_{i}$ and $\vec{v}_{j}$ are the optimization variables.

Plug Eq.(11) and Eq.(12) into Eq.(13) and apply KL distance, then Eq.(13) can be further written as:

$$
O_{1}=\min \left(-\sum_{\left(v_{i}, v_{j}\right) \in E} \omega_{i j} \log \hat{p}_{1}\left(\vec{v}_{i}, \vec{v}_{j}\right)\right) .
$$

For the second-order proximity, the similarity between vertices $v_{i}$ and $v_{j}$ is calculated as the following empirical probability:

$$
p_{2}\left(v_{j} \mid v_{i}\right)=\frac{\omega_{i j}}{\lambda_{i}}
$$

where $\lambda_{i}=\sum_{v_{j} \in N\left(v_{i}\right)} \omega_{i j}$ with $N\left(v_{i}\right)$ being $v_{i}$ 's neighborhood vertex set. Note that $\lambda_{i}$ represents the prestige of vertex $v_{i}$ in the network.

For the second-order proximity, according to LINE, each vertex in the original graph also acts as a "context". Let $\vec{v}_{j}^{\prime}$ denote the embedded vector when $v_{j}$ is treated as "context", the probability of $\vec{v}_{j}^{\prime}$ based on $\vec{v}_{i}$ can be expressed as:

$$
\hat{p}_{2}\left(v_{j} \mid v_{i}\right)=\frac{\exp \left(\vec{v}_{j}^{T} \cdot \vec{v}_{i}\right)}{\sum_{k=1}^{|V|} \exp \left(\vec{v}_{k}^{T} \cdot \vec{v}_{i}\right)}
$$


The goal is to make the conditional distribution $\hat{p}_{2}\left(\cdot \mid v_{i}\right)$ as close as possible to the empirical distribution $p_{2}\left(\cdot \mid v_{i}\right)$. Therefore, based on the second-order proximity, the optimization problem Eq.(10) becomes:

$$
O_{2}=\min \sum_{v_{i} \in V} \lambda_{i} d\left(\hat{p_{2}}\left(\cdot \mid v_{i}\right), p_{2}\left(\cdot \mid v_{i}\right)\right) .
$$

Plug Eq.(15) and Eq.(16) into Eq.(17) and apply KL distance. Therefore, Eq.(17) becomes:

$$
O_{2}=\min \sum_{e_{i j} \in E} \omega_{i j} \log p_{2}\left(v_{j} \mid v_{i}\right) .
$$

B Real-time Distributed Graph Partition and Embedding

\section{Graph Partition}

To facilitate big data applications, we divide the incoming big data into many clusters and then perform graph embedding distributively. The task of dividing a large graph into several subgraphs is a classic problem called graph partition. Most existing graph partition methods are based on vertex partition [20-22], which divide vertices into un-overlapped subgraphs. However, the main drawback of vertex based partitions is that they cannot guarantee balanced graph partitions due to the uncertainty of the degree of each vertex. In this paper, we propose a new edge based graph partition method with the following features:

- It avoids unequal graph partition. In real-time streaming applications, an edge is the basic input unit for graph partition and embedding. Meanwhile, the computational 


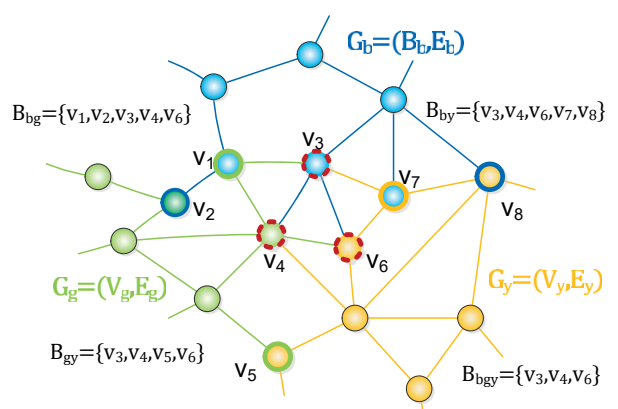

Figure 2: Edge Partition.

complexity of graph embedding depends on the number of edges (rather than vertices) in the subgraph. As a result, our edge based method simplifies the partition process and balances the complexity of the distributed machinery.

- The similarities among vertices (prior to partition) are maximally preserved after graph partition.

- It completely eliminates communication overhead among clusters during the partition process.

For a given graph $G=(V, E)$, all the edges are divided into $K$ different subgraphs $G_{k}=\left(V_{k}, E_{k}\right)$ without overlapping, i.e.,

$$
E=\cup_{k=1}^{K} E_{k} \quad \forall i, j: i \neq j \Rightarrow E_{i} \cap E_{j}=\emptyset,
$$

where $K$ is the pre-set total number of subgraphs. Note that adjacent subgraphs usually have overlapped vertices. We denote $B_{i j}=\left\{v_{k} \mid v_{k} \in V_{i} \cap V_{j}\right\}$ as the overlapped vertex set between subgraph $G_{i}$ and $G_{j}$, where $V_{i}$ and $V_{j}$ are the vertex sets of $G_{i}$ and $G_{j}$ respectively.

Fig. 2 shows three subgraphs $G_{b}, G_{g}, G_{y}$, whose edges are colored in blue, green and yellow respectively. As we can see, $v_{1}$ is connected by both blue and green edges so 
that $v_{1}$ belongs to both $G_{b}$ and $G_{g}$ (i.e., $v_{1} \in B_{b g}$ ). Accordingly, we have $B_{b g}=\left\{v_{1}, v_{2}, v_{3}, v_{4}, v_{6}\right\}, B_{b y}=\left\{v_{3}, v_{4}, v_{6}, v_{7}, v_{8}\right\}$ and $B_{g y}=\left\{v_{3}, v_{4}, v_{5}, v_{6}\right\}$. Meanwhile, vertices $v_{3}, v_{4}$ and $v_{6}$ are shared by all three subgraphs.

In order to have a balanced graph partition, the size of each subgraph (i.e., the number of edges) should be as close as possible to the average size of $|E| / K$. Therefore, we use the standard deviation of the subgraph size to measure the balance of graph partition:

$$
s t d=\sqrt{\frac{\sum_{k=1}^{K}\left(\frac{\left|E_{k}\right|}{|E| / K}-1\right)^{2}}{K}} .
$$

For subgraph $G_{k}$, let $\boldsymbol{Z}^{(k)}, \boldsymbol{Y}^{(k)}, \in \mathbb{R}^{\left|V_{k}\right| \times d}$ be the $d$-dimensional embedded vector sets by subgraph embedding and global embedding, respectively. The objective function for graph partition optimization is:

$$
\min \sum\left\|\boldsymbol{Z}^{(k)}-\boldsymbol{Y}^{(k)}\right\|^{2}
$$

Furthermore, the communication cost among the whole partitions also need to be considered. In order to have a effective performance on the following aggregation process, the communication cost should be as small as possible which is defined as the number of overlapping vertices:

$$
\min \sum_{i} \sum_{j}\left|B_{i j}\right|
$$

where $B_{i j}$ is the overlapping vertex set between two subgraphs $G_{i}$ and $G_{j}$, for $\forall i, \forall j \in$ $[1, K], i \neq j$ as we discussed above.

In [50], author provides an ideally simple solution that: to compute $K$ partitions, $K$ edges are chosen at random and each partition grows around those edges. Then, all 
partitions take control of the edges that are neighbors of those already in control and are not taken by other partitions. All partitions will incrementally get larger and larger until all edges have been taken. However, this method is not practical in real-world cases, since the starting position may influence the size of the partitions. For instance, a partition that starts from the center of the graph will have more space to expand than a partition that starts from the border and/or very close to another partition. Additionally, thus graph partition problem is a NP-hard problem in general $[20,51]$. To overcome this issuer, we propose our greedy one-pass edge partition algorithm consumes the subgraphs in a streaming fashion which means requiring only a single pass. Besides, in order to preserve the original graph similarity for each subgraph, we also consider balanced weights in partitions due to the above analysis that the empirical probability is effected by the total weights. It proceeds as followings:

- Initially, all $K$ subgraphs are open to accept new edges. A subgraph will be considered closed if its capacity (maximally number of edges allowed) is reached.

- An incoming edge $e=(u, v)$ will be assigned to the subgraph who has the minimum weight among all subgraphs containing vertex $u$ or $v$, where the weight of subgraph $G_{k}$ is given by $\sum_{e(u, v) \in E_{k}} \omega_{u v}$.

- In order to balance the number of edges $\left|E_{k}\right|$ in each subgraph $(k=1,2 \ldots K)$, we set a given threshold $t_{e}$, if $\max \left(\left|E_{k}\right|\right)-\min \left(\left|E_{k}\right|\right) \geq t_{e}$, the next incoming edge with the minimum weight over time (from the beginning to the current state) will be assigned to the subgraph with $\min \left(\left|E_{k}\right|\right)$. 


\section{Dynamic Graph Embedding}

In real-time applications, graph data come in a streaming fashion. Therefore, we focus on dynamic graph embedding of parallel subgraphs where the embedded vectors are updated when new edges come in.

For each subgraph, solving optimization problems Eq.(14) and Eq.(18) directly is computationally prohibitive. To reduce the complexity, negative sampling was proposed in $[14,15]$ that transforms Eq.(14) and Eq.(18) to the new problem of jointly maximizing the probabilities of positive samples and minimizing the probabilities of a small number of negative samples. For a given vertex, its positive samples are those vertices directly connected to it, while its negative samples are those vertices without direct connections. Accordingly, the new optimization problem becomes:

$$
O_{3}=\min \left(\sum_{n=1}^{M} E_{v_{n} \sim P_{n}(v)}\left[\psi_{v_{i}, v_{n}}^{-}\right]-\psi_{v_{i}, v_{j}}^{+}\right),
$$

where $\psi_{v_{i}, v_{n}}^{-}=\log \sigma\left(\vec{v}_{n}^{T} \cdot \vec{v}_{i}\right)$ and $\psi_{v_{i}, v_{j}}^{+}=\log \sigma\left(\vec{v}_{j}^{\prime T} \cdot \vec{v}_{i}\right)$ are the probabilities associated with the negative and positive samples, and $\sigma(x)=\frac{1}{1+\exp (-x)}$ is the sigmoid function. Note that negative samples $v_{n}$ are selected according to noise distribution $P_{n}(v)=\left(\frac{f(v)}{F}\right)^{\alpha}$, where $f(v)$ is the weight sum of the selected negative samples, $F=\sum_{v \in V_{\text {neg }}} f(v)$, and $\alpha$ is a smoothing parameter.

To solve Eq.(23), we use the stochastic gradient algorithm (SGD) shown in Algorithm 1. Given an edge $\left(v_{i}, v_{j}\right)$ and $M$ negative samples, the gradient $\frac{\partial O}{\partial \vec{v}_{i}}$ is computed and the embedded vector is updated as: $\vec{v}_{i}=\vec{v}_{i}+\eta \frac{\partial O}{\partial \vec{v}_{i}}$, where $\eta$ is the learning rate.

Algorithm 1 has two major limitations: (1) It is not applicable to streaming graph. Algorithm 1 needs to calculate the noise distribution up front. However, in steaming 


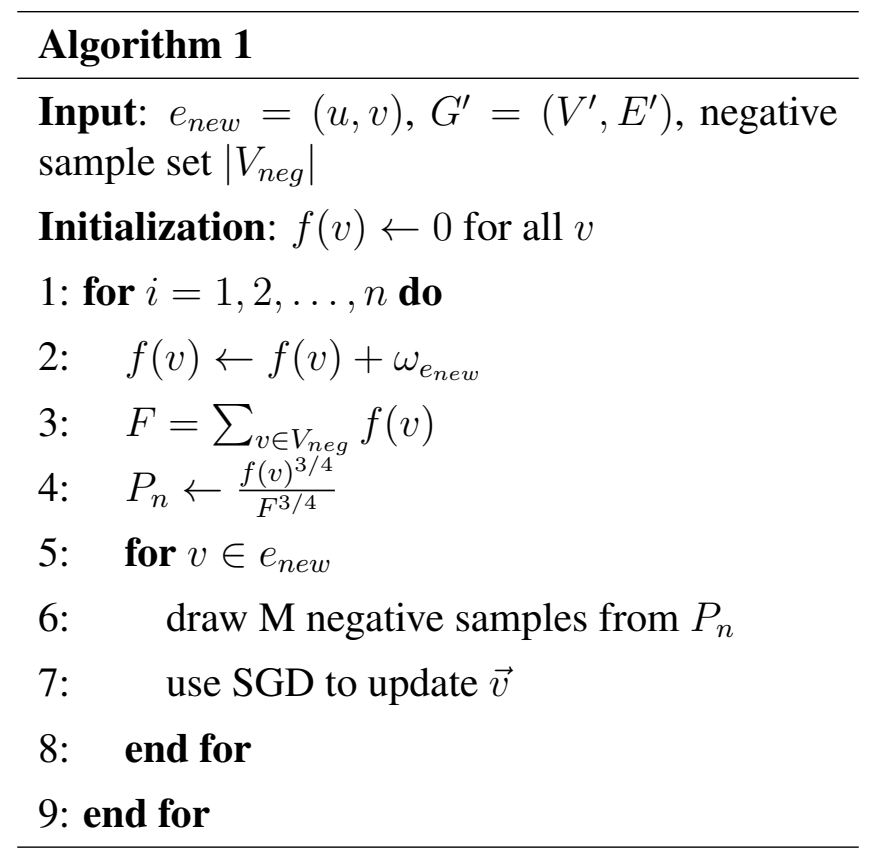

applications, the graph data is only partially available at any given time. (2) The complexity of Algorithm 1 is too high for large scale data sets. Calculating the noise distribution requires a search over the entire vertex set, which will exhaust the computing power and memory of distributed workers. To overcome these two issues, we propose Algorithm 2 to only keep track of the top- $L$ frequent vertices in calculating the noise distribution, based on partially observed graph. In this way, we can handle streaming big data input with significantly reduced complexity.

Remark 1: Algorithm 2 targets for streaming applications, where the top- $L$ frequent vertices and the noise distribution are based on current partial graph and they are constantly updated as more data come in.

Remark 2: The negative sampling method is implemented by a vertex array known as negative table $T$, where the number of copies of vertex $v$ appended into the table is proportional to $P_{n}(v)$. Then, a negative sample is generated by uniformly selecting an element from the negative table. In Algorithm 2, $\tau$ is the maximum size of the negative 


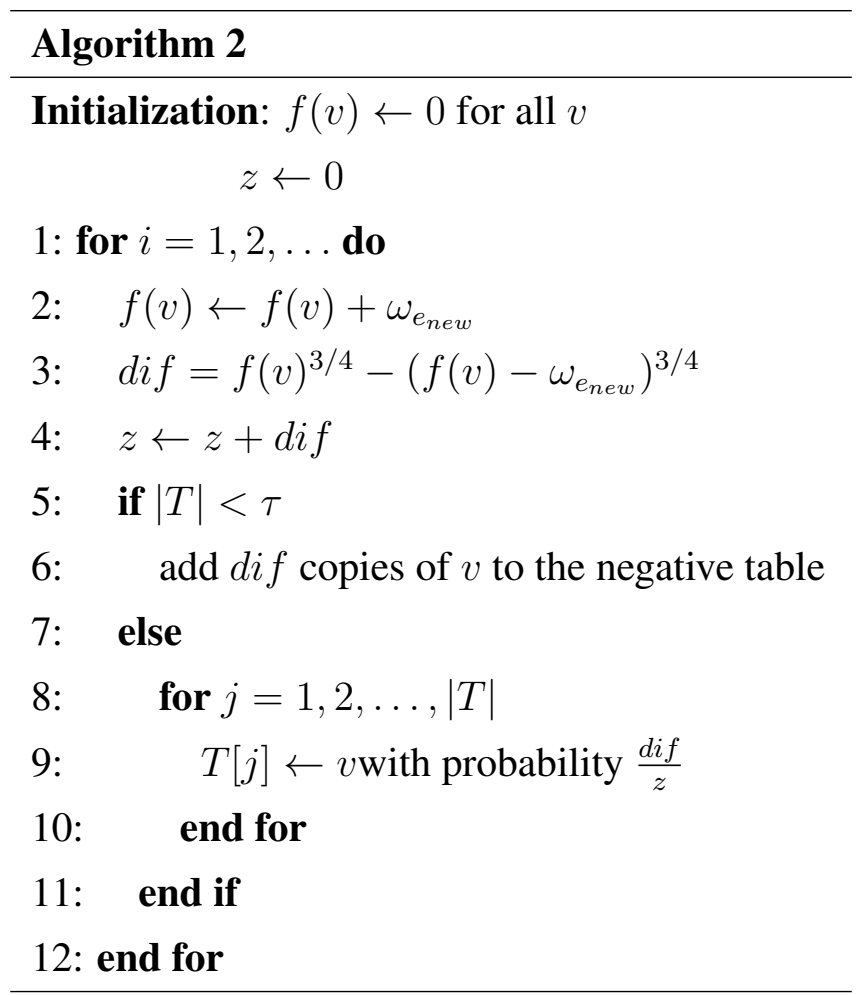

table. When $|T|<\tau$, dif copies of vertex $v$ can be added directly into the table. When $|T|=\tau$, element $T[j]$ will be replaced by vertex $v$ with probability $\frac{d i f}{z}$.

Remark 3: The complexities of Algorithm 1 and 2 are respectively $\mathcal{O}(|E|)$ and $\mathcal{O}(\tau)$, where $\tau<<|E|$.

\section{Heuristic Global Aggregation}

The last step of RTDGE is global aggregation of the distributively embedded subgraphs. The basic idea of our unsupervised global aggregation is to find a feasible global vector space which mapping from multiple local embedding sub-spaces by utilizing the overlapped vertex set $B$.

Assume vertex $v_{m}$ belongs to multiple subgraphs, e.g., $G_{i}$ and $G_{j}$ indicated by $v_{m} \in B_{i j} ; \boldsymbol{Z}^{(i)}=F\left(G_{i}\right)$ and $\boldsymbol{Z}^{(j)}=F\left(G_{j}\right)$ are the local embedded vector spaces of $G_{i}$ 
and $G_{j}$ respectively. As a result, the local embedded vectors of $v_{m}$ in subgraph $G_{i}$ and $G_{j}$ can be expressed as $\boldsymbol{z}_{m}^{(i)}$ and $\boldsymbol{z}_{m}^{(j)}$ respectively. If there exists a mapping function $h\left(\boldsymbol{z}_{m}^{(i)}, \boldsymbol{z}_{m}^{(j)}\right) \longrightarrow \boldsymbol{y}_{m}$ for the overlapped vertex $v_{m}$, we can use this function to map the entire subspace $\boldsymbol{Z}^{(i)}$ and $\boldsymbol{Z}^{(j)}$ into a global vector space $\boldsymbol{Y}$ :

$$
h\left(\boldsymbol{Z}^{(i)}, \boldsymbol{Z}^{(j)}\right) \stackrel{h\left(\boldsymbol{z}_{m}^{(i)}, \boldsymbol{z}_{m}^{(j)}\right)}{\longrightarrow} \boldsymbol{Y}
$$

We design a low complexity unsupervised global aggregation algorithm by applying the linear transformation method. The designed algorithm which is simple but effective includes two processes: (1) normalization and (2) combination. Specifically, we first find the overlapped vertex set $B_{\text {all }}=V_{1} \cap V_{2} \cap \ldots \cap V_{K}$ among all subgraphs. Then, for each vertex $v_{m} \in B_{\text {all }}$, we normalize its local embedded vector $\boldsymbol{z}_{m}^{i}=\left[z_{m}^{i}(1), z_{m}^{i}(2), \ldots, z_{m}^{i}(d)\right]$ in every cluster $i$ as:

$$
\boldsymbol{z}_{m}^{(i)^{\prime}}=\left[\frac{z_{m}^{i}(1)-e_{m}^{i}}{\sigma_{m}^{(i)}}, \frac{z_{m}^{i}(2)-e_{m}^{i}}{\sigma_{m}^{(i)}}, \ldots, \frac{z_{m}^{i}(d)-e_{m}^{i}}{\sigma_{m}^{(i)}}\right]
$$

where $e_{m}^{i}=\frac{\sum z_{m}^{i}(1)+z_{m}^{i}(2)+\cdots+z_{m}^{i}(d)}{d}$ is the mean value of $\boldsymbol{z}_{m}^{i}$ and $\sigma_{m}^{(i)}{ }^{2}$ is the variance.

After normalization process, the combination process is conducted to find/form the global space by combining the normalized embedded vectors in different local clusters of each overlapped vertex. While various combination method can be applied, we adopt the average operator. By using Eq.(25), the average of the normalized vectors of vertex $v_{m}$ is calculated as: $\boldsymbol{z}_{m}^{\prime}=\frac{\sum_{i=1}^{K} \boldsymbol{z}_{m}^{(i)^{\prime}}}{K}$. Therefore, the mapping function for vertex $v_{m}$ can be expressed as: $z_{m}^{i}=h_{m}\left(\boldsymbol{z}_{m}^{(i)}, \boldsymbol{z}_{m}^{(j)}\right)$. Then, the global standard embedded vector is calculated as:

$$
\boldsymbol{z}^{(\text {all })}=\frac{\sum_{m \in B_{\text {all }}} \boldsymbol{z}_{m}^{\prime}}{\left|B_{\text {all }}\right|}
$$


TABLE 2

Data sets used for experiments in Section IV.

\begin{tabular}{|c|c|c|}
\hline Name & Edges \# & Vertices \# \\
\hline \hline AstroPH & 196,972 & 17,903 \\
\hline USRoad & $3,083,796$ & $2,541,898$ \\
\hline Les Miserables & 254 & 77 \\
\hline BlogCatalog & 333,983 & 10,312 \\
\hline Wikipedia & 184,812 & 4,777 \\
\hline YouTube & $4,945,382$ & $1,138,499$ \\
\hline
\end{tabular}

Furthermore, the local graph embedded vector space $Z^{(i)}$ can be mapped to a partial global vector space $\boldsymbol{y}_{i}^{\prime}$ as:

$\boldsymbol{y}_{i}^{\prime}=\boldsymbol{Z}^{(i)}-$ dist $^{(i)}$,
where $d i s t^{(i)}=\frac{\sum_{v_{m} \in V_{i} \cap B_{\text {all }}}\left(\boldsymbol{z}_{m}^{(i)}-\boldsymbol{z}^{(a l l)}\right)}{\left|V_{i} \cap B_{\text {all }}\right|} \quad \forall v_{m} \in V_{i} \cap B_{(\text {all })}$.

Finally, the global feature representation $\boldsymbol{Y}$ can be produced as: $\boldsymbol{Y}=\left[\boldsymbol{y}_{1}^{\prime}, \boldsymbol{y}_{2}^{\prime}, \ldots, \boldsymbol{y}_{K}^{\prime}\right]$

C Experiments

1 System Setup

In order to implement our RTDGE algorithm and evaluate its real-world performance, we develop a real-time distributed graph embedding platform based on Apache Kafka and Apache Storm. The system consists of three servers: each server has 8GB memory, quad-core Intel Xeon CPU and 500GB disk space. The connection among servers are constructed by Apache Zookeeper. Unlike GraphX that is only built upon Apache Spark, in our platform the streaming data are retrieved by Apache Kafka and 


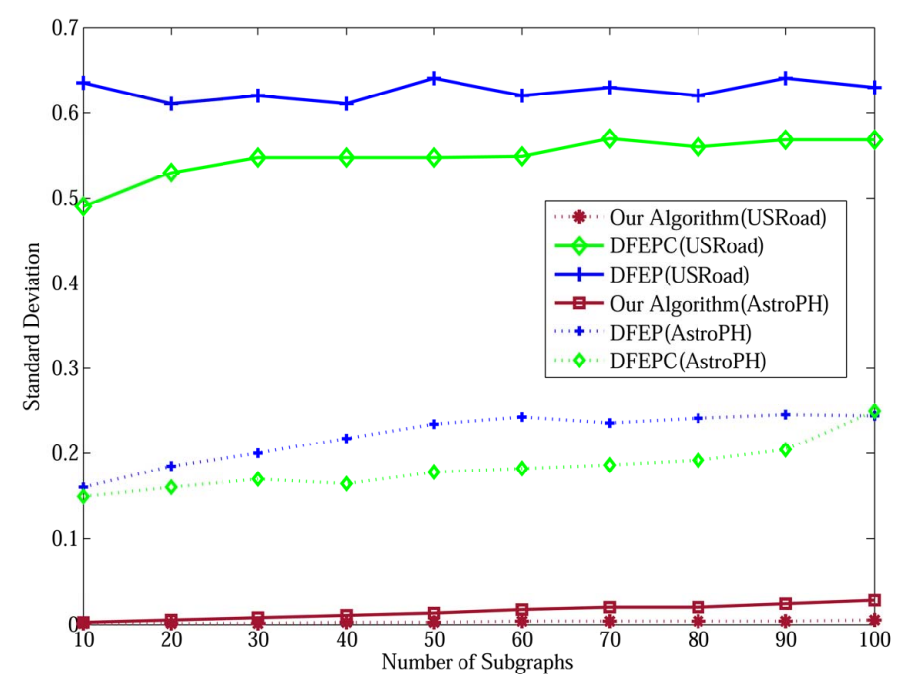

Figure 3: Subgraph Size Stand Deviation Evaluation.

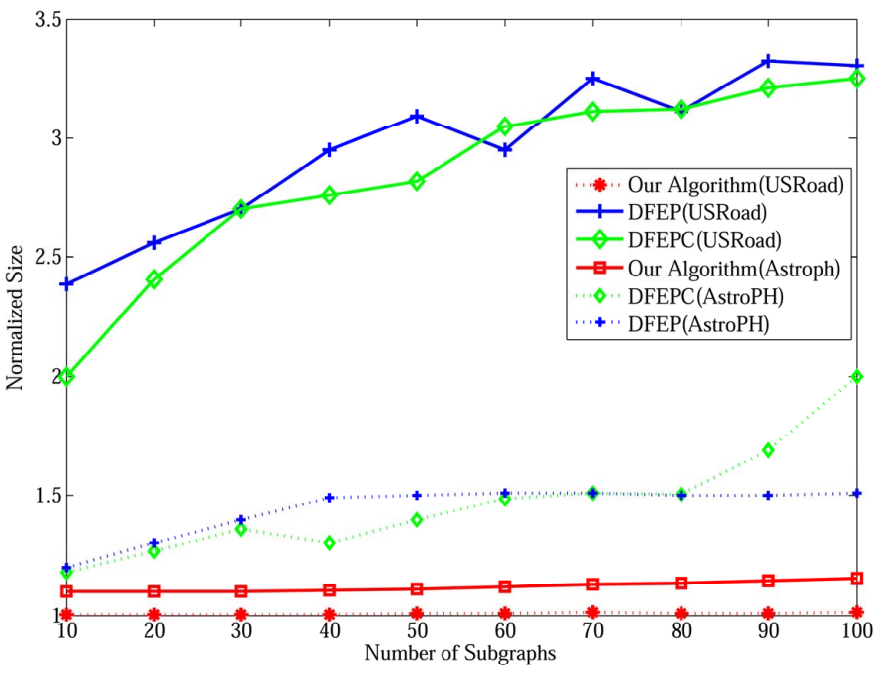

Figure 4: Maximum Subgraph Size.

distributed into multiple brokers according to our edge partition algorithm in Section III. A typical Apache Kafka consists of a producer and a consumer. Multiple producers and consumers can publish and retrieve data at the same time. A data message is defined as a topic, which can be divided into multiple partitions. One or more partitions can be stored in each broker. The data in each broker can be consumed by one or multiple Apache Storm clusters including one Apache Storm Nimbus and multiple Apache Storm Supervisors. The data sets we used for experiments are summarized in TABLE 2. 


\section{Graph Partition}

In Fig. 3 and Fig. 4, we compare the performance of our approach with two other methods. Distributed funding-based edge partitioning (DFEP) is an edge partition algorithm based on the concept that every vertex has certain amount of money to buy assigned edges, and DFEPC is another version of DFEP with different parameters. In [50], the authors show that DFEP and DFEPC outperform METIS using the following two data sets: (1) AstroPH [52] is a weighted network of co-authorships among scientists posting preprints on High-Energy Theory E-Print Archive between Jan 1, 1995 and December 31, 1999. It has 17,903 vertices and 196, 972 edges. (2) USRoad is a road network in Pennsylvania where nodes represent the intersections and endpoints while edges represent roads. The network has 2, 541, 898 nodes and 3, 083, 796 edges. The metrics we used to evaluate the performance are: (i) Standard deviation of subgraph size by Eq.(20); (ii) The size of the largest subgraph normalized by the average subgraph size. From Fig. 3 and

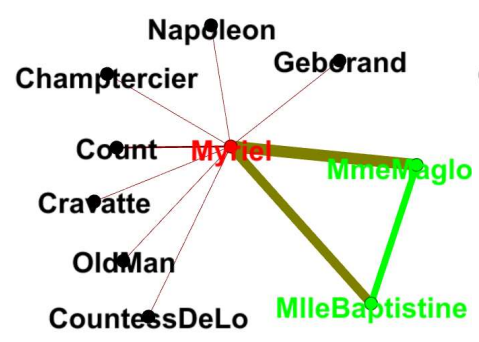

(a)

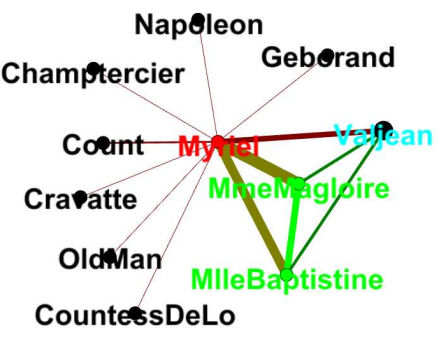

(b)

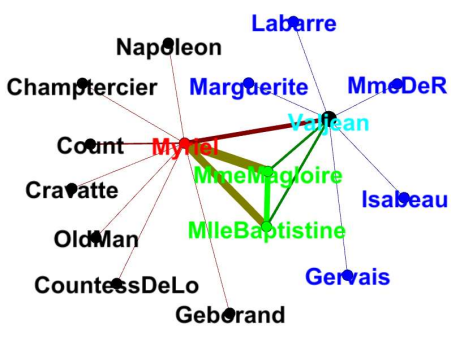

(c)

Figure 5: Original Les Miserables network at (a) $T=t_{i}$, (b) $T=t_{j}$, (c) $T=t_{k}$.

Fig. 4, we can see that both the variance of the subgraph size and the size of the largest subgraph increase with the number of subgraphs for all partition methods. Apparently, our algorithm outperforms others with a large margin in all cases. In particular, when $K$ is increased from 10 to 100 , our algorithm is able to keep the standard deviation close to zero 
and the size of the largest subgraph is very close to one (i.e., the average subgraph size).

\section{Dynamic Graph Embedding}

Since our dynamic graph embedding is designed for streaming data input, it is capable of revealing the evolution of network structure over time. In this section, we use the famous novel Les Miserables by Victor Hugo as a case study to illustrate the effectiveness of our dynamic graph embedding. The Les Miserables network contains co-appearances of 77 characters. In graph representation, a node represents a character and an edge means that these two characters appear in the same chapter of the book. There are 254 edges in this network. The weight of each link indicates how often such a co-appearance occurs. We embed the Les Miserable network into a 3 dimensional vector space and monitor the embedded results at three different time instances:

- $T=t_{i}$, the network has ten characters.

- $T=t_{j}$, the network has eleven characters (the main character Valjean appears).

- $T=t_{k}$, the network has sixteen characters.

When $T=t_{i}, t_{j}, t_{k}$, Fig. 5 shows the original Les Misrable graph. Fig. 6 consists of the visualizations of the embedded results. As we can see in Fig. 12a, the embedded nodes are clustered into three different groups. It turns out that the isolated red node (Node 0) is character Myriel who plays an important role at the beginning. Myriel is also shown in Fig. 5a as the center node with an unique connection pattern. Therefore, it is reasonable to be isolated in the embedded space. Furthermore, the green nodes are Node 2 (MlleBaptistine) and Node 3 (MmeMaglorie), who have the same connection pattern (i.e., connected with 
each other and only linked with Node 0) distinguished from others. As a result, they are located in the same position after graph embedding. We also see that the remaining black nodes are close since they are similar by the first-order proximity.

When $T=t_{j}$, Fig. 12b illustrates the embedded results. A new Node 11 (Valjean) is embedded closely to Node 2 and Node 3. In Fig. 5b, Node 11 is more similar to Node 2 and 3 by the first-order proximity. However, by the second-order proximity, Node 11 clearly has different connection pattern from Node 2 or 3 , as shown by their distances in Fig. 12b. On the other hand, the black nodes are almost overlapped because their connection patterns are very similar.

When $T=t_{k}$, the graph embedding results under first-order proximity and secondorder proximity are shown in Fig. 6c and 6c, respectively. In Fig. 6c, there are two isolated nodes, Node 0 (Myriel, colored in red) and Node 11 (Valjean, colored in cyan). Besides, there are two node clusters: one contains black stars which are existing characters; the other cluster contains blue circles which are new characters. According to our previous analysis, nodes in the same cluster should share similar connection pattern. As shown in Fig. 5c, new nodes are only connected with Node 11 (Valjean).

\section{Multi-label Classification}

In multi-label classification setting, each node is assigned one or more labels from a finite set $L$. During the training phase, we observe a certain fraction of nodes with their labels. The objective is to predict the labels of the remaining nodes, which is challenging when $L$ is large. In our experiments, we evaluate the performance of our RTDGE by comparing it with the following algorithms: 

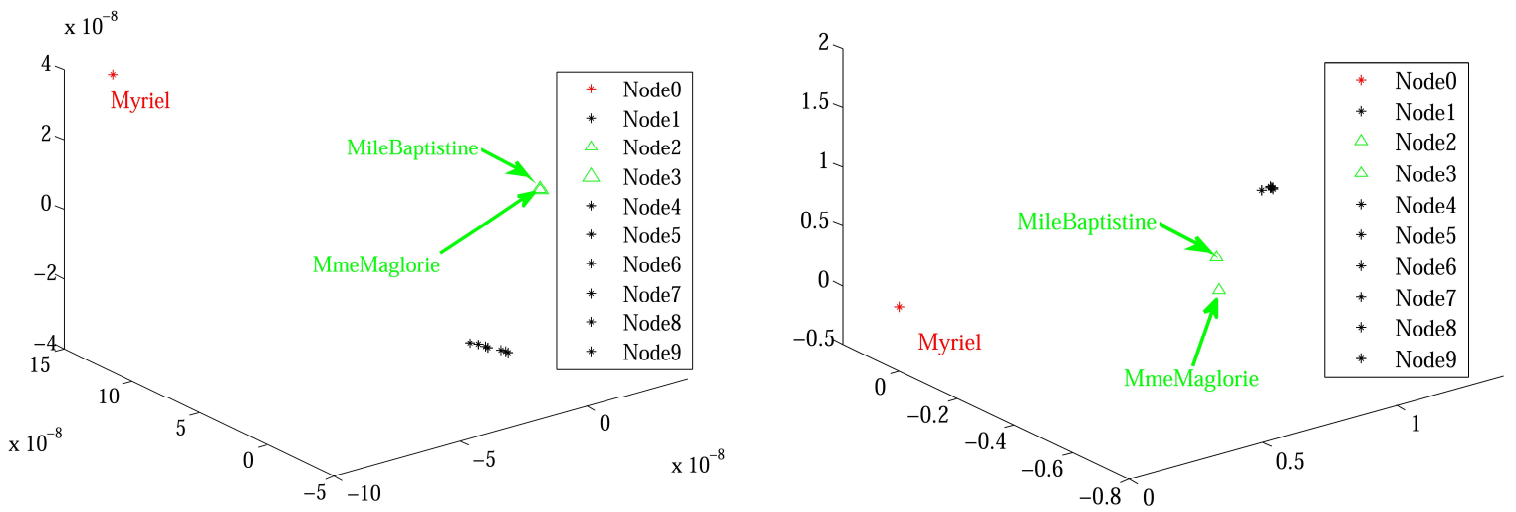

(a) 3D Graph embedding before Valjean appears $\left(T=t_{i}\right)$
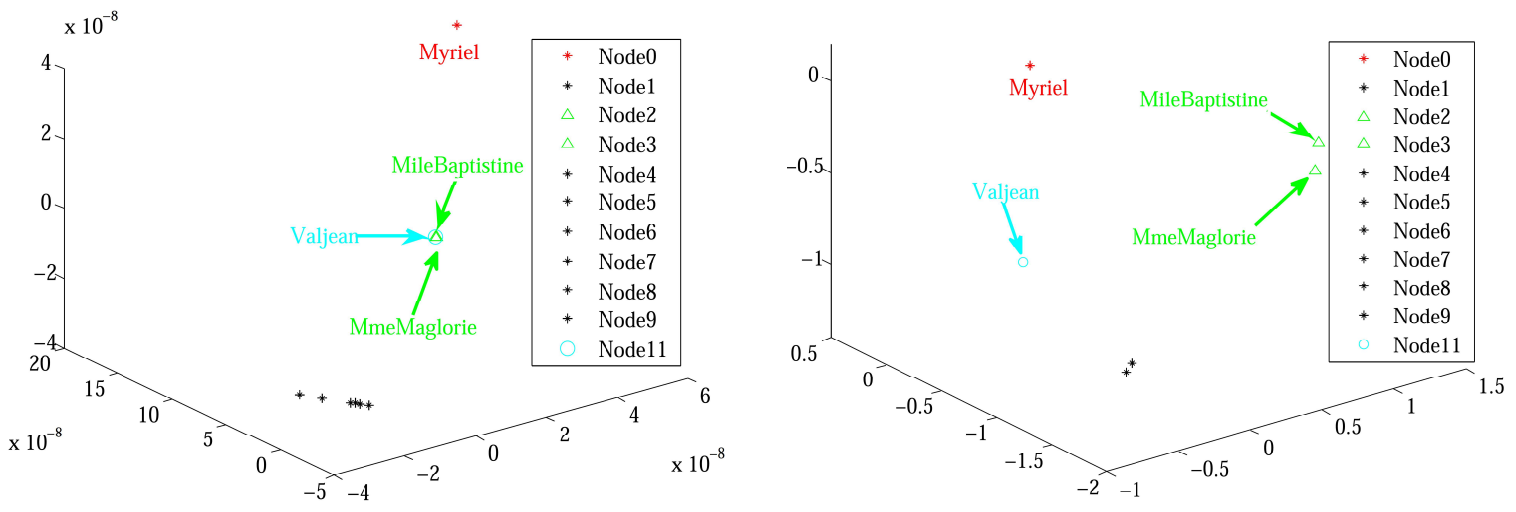

(b) 3D Graph embedding when Valjean appears $\left(T=t_{j}\right)$
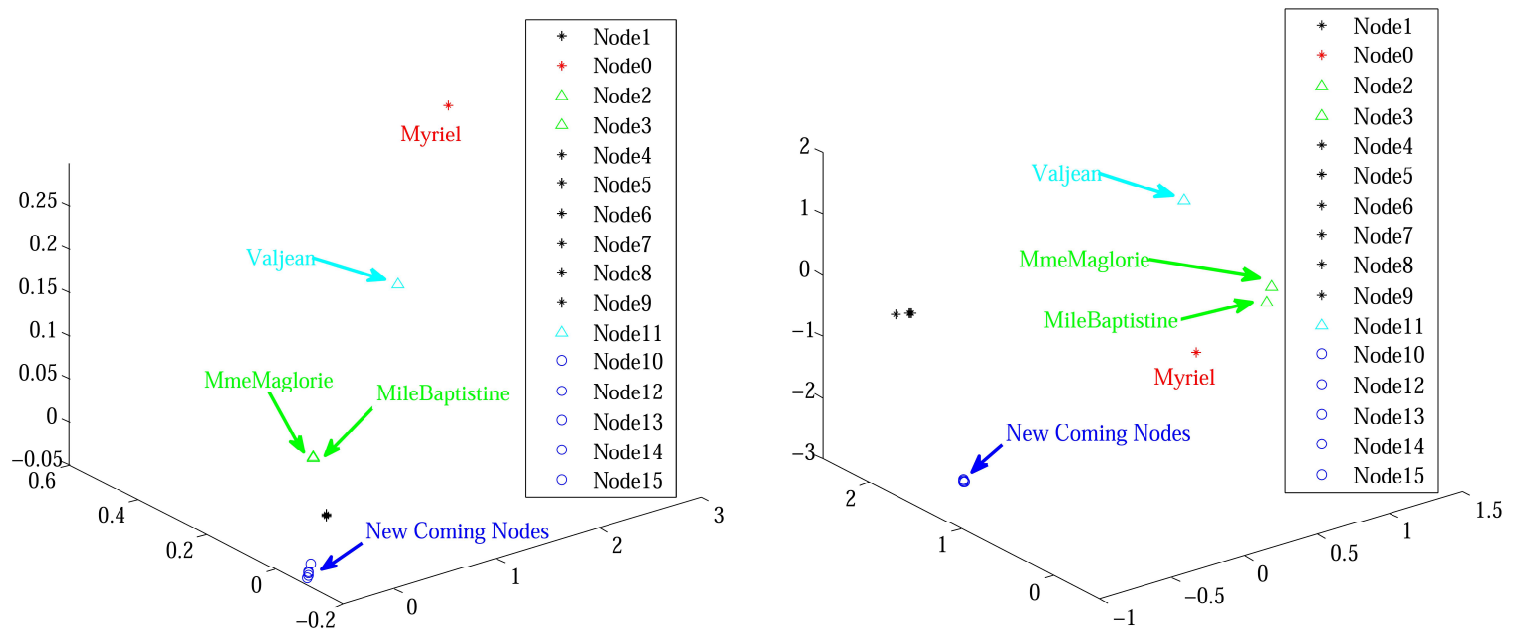

(c) 3D Graph embedding after Valjean appears $\left(T=t_{k}\right)$

Figure 6: Dynamic graph embedding results. 
TABLE 3

Multi-label classification results of BlogCatalog.

\begin{tabular}{|c|c|c|c|c|c|c|c|c|c|c|}
\hline Metric & Algorithm & $10 \%$ & $20 \%$ & $30 \%$ & $40 \%$ & $50 \%$ & $60 \%$ & $70 \%$ & $80 \%$ & $90 \%$ \\
\hline \hline Macro-F1 & SpectralClustering & 3.01 & 3.21 & 3.49 & 4.01 & 4.22 & 4.50 & 4.81 & 5.01 & 5.11 \\
\cline { 2 - 10 } & DeepWalk & 5.03 & 7.53 & 9.03 & 10.03 & 11.03 & 11.33 & 11.63 & 11.93 & 12.63 \\
\cline { 2 - 10 } & LINE1st & 13.65 & 13.69 & 13.77 & 13.57 & 14.06 & 13.51 & 13.31 & 14.69 & 13.27 \\
\cline { 2 - 10 } & LINE2nd & 13.88 & 13.75 & 13.54 & 13.88 & 13.71 & 13.37 & 12.91 & 13.18 & 13.08 \\
\cline { 2 - 10 } & RTDGE1st & 14.01 & 14.08 & 14.13 & 14.19 & 14.25 & 14.39 & 14.61 & 14.72 & 14.76 \\
\cline { 2 - 10 } & RTDGE2nd & 13.79 & 13.81 & 13.71 & 13.92 & 13.78 & 14.02 & 13.96 & 14.13 & 13.95 \\
\hline \hline Micro-F1 & SpectralClustering & 16.15 & 17.51 & 17.91 & 18.11 & 18.99 & 19.76 & 20.87 & 21.03 & 22.19 \\
\cline { 2 - 10 } & DeepWalk & 16.53 & 18.73 & 20.13 & 20.83 & 21.53 & 21.83 & 22.03 & 22.03 & 22.5300 \\
\cline { 2 - 9 } & LINE1st & 22.04 & 18.34 & 23.12 & 21.90 & 23.16 & 22.95 & 24.12 & 24.34 & 24.43 \\
\cline { 2 - 9 } & LINE2nd & 15.25 & 12.97 & 13.94 & 14.22 & 14.65 & 14.04 & 13.18 & 15.01 & 15.29 \\
\cline { 2 - 9 } & RTDGE1st & 24.57 & 24.77 & 24.76 & 24.85 & 24.24 & 24.71 & 24.21 & 24.29 & 24.66 \\
\cline { 2 - 9 } & RTDGE2nd & 24.21 & 24.25 & 24.10 & 24.44 & 24.23 & 24.58 & 24.49 & 24.76 & 24.29 \\
\hline \hline
\end{tabular}

- Spectral clustering [53] is a matrix factorization approach where the top $d$ eigenvectors of the normalized Laplacian matrix are used as nodes' feature vector representations.

- DeepWalk [16] is a classic graph embedding approach, where random walks are generated from each vertex to obtain the contextual information of the network.

- LINE [27] contains an edge sampling method that improves the computational efficiency over the traditional SGD method. It has two proximities: LINE1st and LINE2nd.

BlogCatalog: We first use BlogCatalog dataset as our input graph. It is a network of social relationships of the bloggers publicly available on BlogCatalog website (http://blogcatalog.com). There are 10, 312 users and 333,983 edges in the network. Each edge represents the friendship between two users. To accomplish the goal of classification, the embedded vectors are input to a one-vs-all logistic regression classifier with L2 
regularization. The well-known Macro-F1 and Micro-F1 scores are used as the performance metric.

TABLE 3 shows the MicroF1 and Macro-F1 scores with different percentage of train data, which is used to train the L2 logistic regression model with $\lambda=1$. The results are averaged over 10 different trials. Apparently, our algorithm achieves higher Micro-F1 scores than other methods, especially under the second-order proximity. This is because: (1) We use adaptive $N$-Gram Negative method instead of the fixed N-Gram Negative (i.e., $N=5$ ) method used by LINE. (2) Unlike LINE which uses negative samples from neighboring nodes, we carefully consider each vertex's connection pattern to avoid using neighboring nodes as negative samples and guarantee all negative samples are selected according to the noise distribution. For the Macro-F1 score, our algorithm is able to achieve almost the same performance as LINE.

\section{TABLE 4}

Multi-label classification results of Wikipedia.

\begin{tabular}{|c|c|c|c|c|c|c|c|c|c|c|}
\hline Metric & Algorithm & $10 \%$ & $20 \%$ & $30 \%$ & $40 \%$ & $50 \%$ & $60 \%$ & $70 \%$ & $80 \%$ & $90 \%$ \\
\hline \hline Macro-F1 & SpectralClustering & 4.01 & 4.02 & 4.03 & 4.11 & 4.51 & 4.69 & 4.78 & 4.91 & 5.01 \\
\cline { 2 - 11 } & DeepWalk & 8.91 & 10.02 & 11.09 & 12.14 & 12.51 & 13.71 & 15.88 & 17.09 & 22.46 \\
\cline { 2 - 10 } & LINE1st & 47.05 & 47.18 & 46.81 & 46.93 & 46.68 & 46.98 & 46.98 & 46.24 & 47.27 \\
\cline { 2 - 10 } & LINE2nd & 47.06 & 47.02 & 46.99 & 46.83 & 464.57 & 47.09 & 47.09 & 46.52 & 46.79 \\
\cline { 2 - 10 } & RTDGE1st & 47.05 & 47.18 & 46.89 & 46.95 & 47.4 & 47.08 & 46.96 & 46.75 & 47.51 \\
\cline { 2 - 10 } & RTDGE2nd & 47.12 & 47.04 & 47.16 & 47.28 & 47.08 & 47.32 & 47.35 & 47.23 & 47.15 \\
\hline \hline Micro-F1 & SpectralClustering & 41.01 & 40.50 & 40.31 & 40.22 & 40.28 & 40.27 & 40.17 & 40.06 & 38.51 \\
\cline { 2 - 9 } & DeepWalk & 46.49 & 48.27 & 51.12 & 52.17 & 52.35 & 52.25 & 52.26 & 52.25 & 52.24 \\
\cline { 2 - 9 } & LINE1st & 63.99 & 64.12 & 63.77 & 63.89 & 63.65 & 63.92 & 63.89 & 63.23 & 64.17 \\
\cline { 2 - 9 } & LINE2nd & 28.73 & 26.71 & 22.31 & 23.93 & 23.49 & 30.96 & 26.19 & 32.94 & 43.23 \\
\cline { 2 - 9 } & RTDGE1st & 63.92 & 63.95 & 63.79 & 63.89 & 64.31 & 64.02 & 63.75 & 63.71 & 64.38 \\
\cline { 2 - 9 } & RTDGE2nd & 64.06 & 63.96 & 63.94 & 63.79 & 63.55 & 64.03 & 64.02 & 64.14 & 63.74 \\
\hline \hline
\end{tabular}

Wikipedia: This is a co-occurrence network of words appearing in the first million bytes of Wikipedia. There are 40 different labels which represent the Part-of-Speech tags by using 
the Standard POS-Tagger. The network contains 4, 777 nodes and 184, 812 edges.

TABLE 4 illustrates the multi-label classification results. For the Macro-F1 score metric, our algorithm outperforms LINE and achieves the highest score at the end point. For the first-order proximity, our Micro-F1 score has $60 \%$ gain over the spectral clustering algorithm. For the second-order proximity, our Micro-F1 score has $55 \%$ gain over the spectral clustering method. Compared to LINE, RTDGE also performs better under both first-order and second-order proximity.

TABLE 5

Multi-label classification results of YouTube.

\begin{tabular}{|c|c|c|c|c|c|c|c|c|c|c|c|}
\hline Metric & Algorithm & $1 \%$ & $2 \%$ & $3 \%$ & $4 \%$ & $5 \%$ & $6 \%$ & $7 \%$ & $8 \%$ & $9 \%$ & $10 \%$ \\
\hline \hline Micro-F1 & SpectralClustering & 24.97 & 26.48 & 27.25 & 27.87 & 28.31 & 28.68 & 29.01 & 29.21 & 29.36 & 29.63 \\
\cline { 2 - 11 } & DeepWalk & 39.68 & 41.78 & 42.78 & 43.55 & 43.96 & 44.31 & 44.61 & 44.89 & 45.06 & 45.23 \\
\cline { 2 - 11 } & LINE & 40.20 & 42.70 & 43.94 & 44.71 & 45.19 & 45.55 & 45.87 & 46.15 & 46.33 & 46.43 \\
\cline { 2 - 11 } & RTDGE10 & 40.68 & 43.58 & 46.39 & 47.63 & 48.87 & 49.70 & 50.64 & 50.78 & 50.86 & 50.74 \\
\cline { 2 - 11 } & RTDGE30 & 44.32 & 44.51 & 44.53 & 44.66 & 44.96 & 45.27 & 5.31 & 45.84 & 48.20 & 52.03 \\
\cline { 2 - 11 } & RTDGE50 & 42.22 & 43.35 & 44.07 & 44.48 & 44.71 & 44.90 & 45.14 & 45.41 & 45.53 & 45.54 \\
\hline \hline Macro-F1 & SpectralClustering & 11.01 & 13.55 & 14.93 & 15.90 & 16.45 & 16.93 & 17.38 & 17.64 & 17.80 & 18.09 \\
\cline { 2 - 11 } & DeepWalk & 28.39 & 30.96 & 32.28 & 33.43 & 33.92 & 34.32 & 34.83 & 35.27 & 35.54 & 35.86 \\
\cline { 2 - 11 } & LINE & 29.85 & 31.93 & 33.96 & 35.46 & 36.25 & 36.90 & 37.48 & 38.10 & 38.46 & 38.82 \\
\cline { 2 - 10 } & RTDGE10 & 37.8800 & 38.0300 & 38.1000 & 38.1400 & 38.1600 & 38.2100 & 38.3100 & 38.4900 & 38.8000 & 39.2500 \\
\cline { 2 - 10 } & RTDGE30 & 38.1094 & 38.1476 & 38.1501 & 38.1755 & 38.2358 & 38.2962 & 38.3051 & 38.4099 & 38.8742 & 39.6300 \\
\cline { 2 - 10 } & RTDGE50 & 37.2082 & 37.2273 & 37.2297 & 37.3355 & 37.3498 & 37.4614 & 37.4800 & 37.5334 & 37.7235 & 38.4010 \\
\hline \hline
\end{tabular}

YouTube: YouTube network is much sparser than other social networks. The dataset has 4,945,382 edges and 1,138,499 nodes. The results are summarized in TABLE 5. We divide the original large-scale graph into 10, 30 and 50 partitions, respectively. We can see that the classification accuracy remains stable with different number of partitions. For the Micro-F1 score, our algorithm has nearly $20 \%$ and $10 \%$ gains over DeepWalk and LINE, respectively. For the Macro-F1 score, our algorithm also outperforms other approaches. 


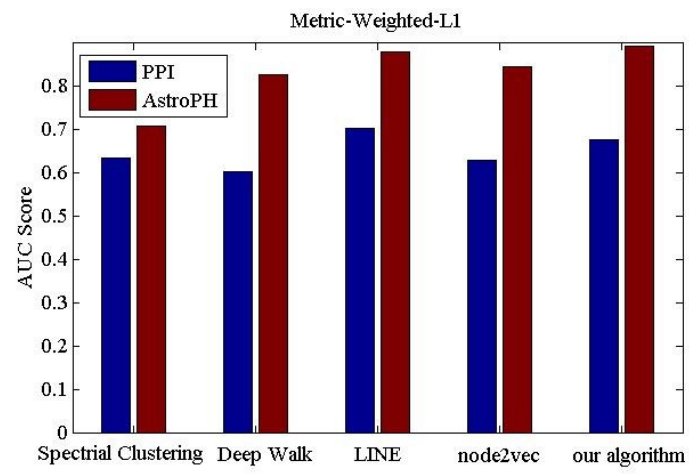

Figure 7: Link prediction results using weighted-L1 operator.

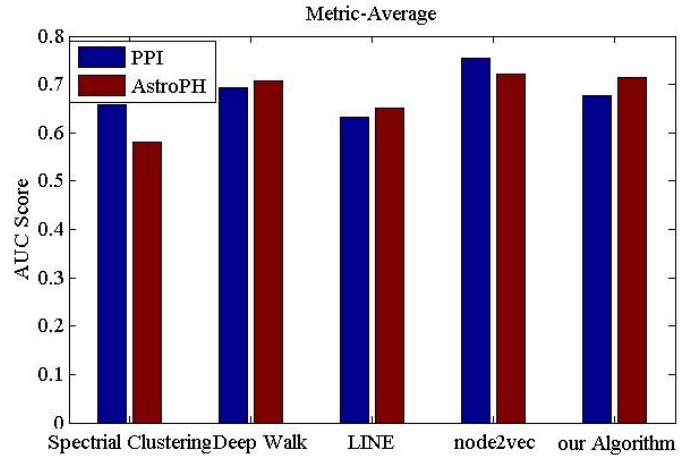

Figure 8: Link prediction results using weighted-L1 operator. 


\section{Link Prediction}

In social network, link prediction aims to predict the potential relations (i.e., missing edges) among existing nodes when only fractional edges are known. The positive samples are generated by removing $50 \%$ edges randomly, while the graph remains connected. To obtain negative samples, the same number of unconnected node pairs from the graph are selected. The following datasets are used for link prediction:

- Protein-Protein Interactions (PPI) network contains 19,706 nodes and 390,633 edges, where each node represents one type of proteins and each edge is the biological interaction between the pair of proteins.

- AstroPH is a weighted network of co-authorships among scientists posting preprints on High-Energy Theory EPrint Archive between Jan 1, 1995 and December 31, 1999. It has 17,903 vertices and 196,972 edges.

We follow the same edge feature learning settings as node2vec. Assume embedded vectors $\vec{v}_{i}, \vec{v}_{j}$ are the feature representations of node $v_{i}$ and $v_{j}$, the edge feature $g\left(v_{i}, v_{j}\right)$ between node $v_{i}$ and $v_{j}$ can be represented as: (1) Average operator: $g\left(v_{i}, v_{j}\right)=\frac{\vec{v}_{i}+\vec{v}_{j}}{2} ;$ (2) WeightedL1 operator: $g\left(v_{i}, v_{j}\right)=\left|\vec{v}_{i}-\vec{v}_{j}\right|$. By applying the logistic regression, the results are shown in Fig. 7 and Fig. 8. We can see that our algorithm has the best performance for AstroPH dataset. For PPI dataset, our RTDGE achieves similar AUC score as LINE with significantly reduced runtime.

TABLE 6 shows the runtime of all algorithms. Clearly, our RTDGE is the fastest algorithm. Specifically, we implement all algorithms in Java on a single machine with 32GB memory, AMD Threadripper 16-core CPU and 1TB SSD. Remarkably, our RTDGE 
TABLE 6

Performance evaluation w.r.t. dimensionality.

\begin{tabular}{|c|c|}
\hline Algorithm & Runtime \\
\hline SpectralClustering & $2.96 \mathrm{hr}$ \\
\hline DeepWalk & $16.6 \mathrm{hr}$ \\
\hline LINE1 st & $2.44 \mathrm{hr}$ \\
\hline LINE2nd & $2.55 \mathrm{hr}$ \\
\hline RTDGE1 st & $0.46 \mathrm{hr}$ \\
\hline RTDGE2nd & $0.51 \mathrm{hr}$ \\
\hline
\end{tabular}

reduces the runtime by at least one hour comparing with other algorithms. It is worth noting that, even with 32GB memory, LINE still suffers from out-of-memory errors.

6 Scalability

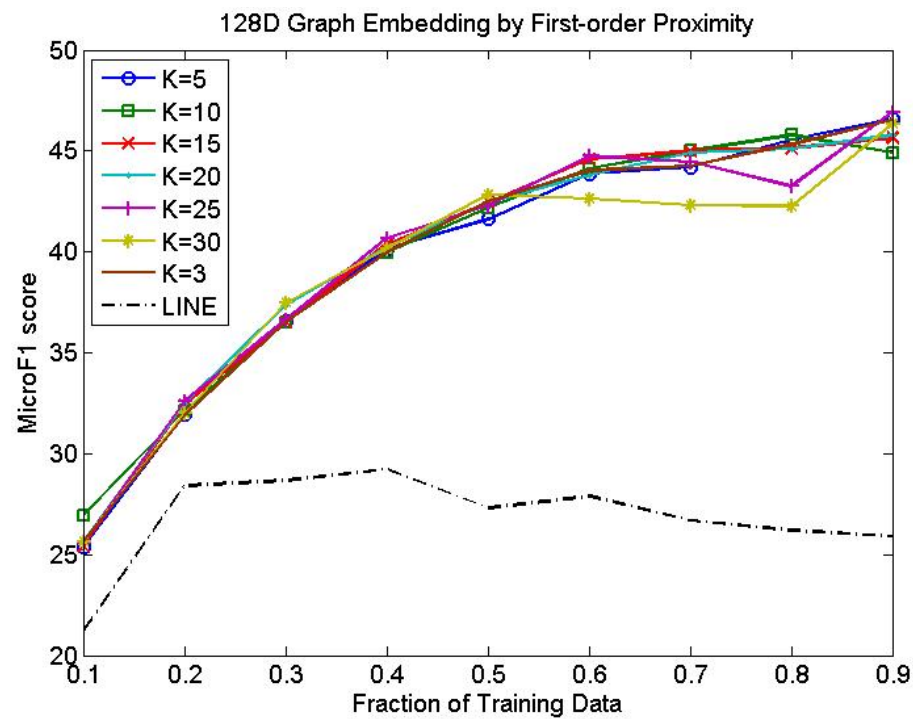

Figure 9: Micro-F1 Performance evaluation w.r.t. \# computing nodes.

The Number of Distributed Processors: We evaluate the Micro-F1 score and MacroF1 score for the multi-label classification application with different numbers of distributed computing nodes. The results are illustrated in Fig. 9 and Fig. 10. We can see that 


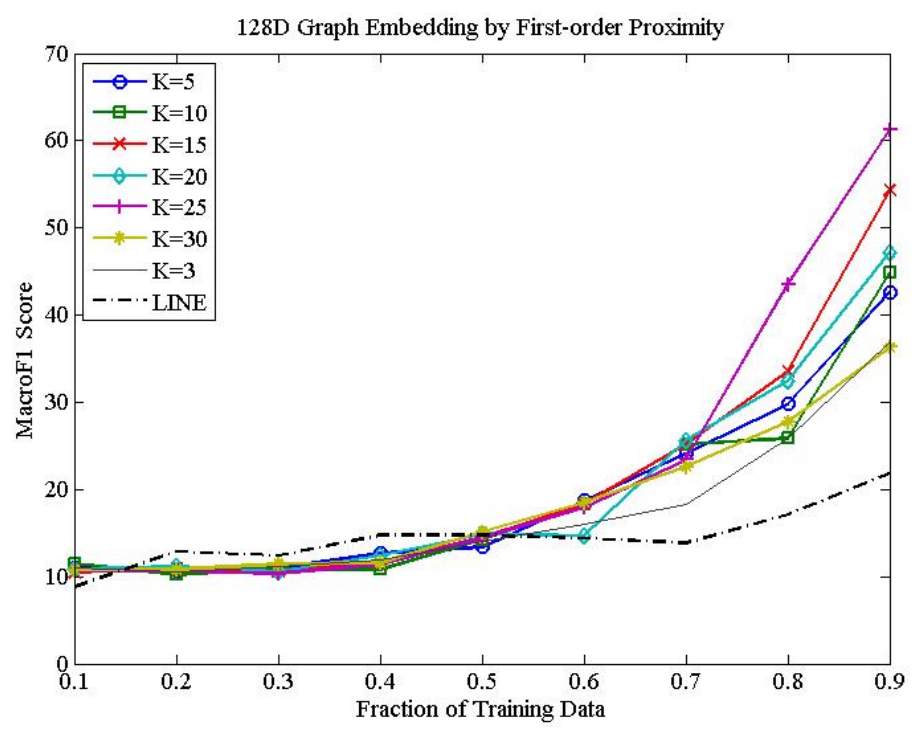

Figure 10: Macro-F1 Performance evaluation w.r.t. \# computing nodes.

our RTDGE is able to hold the performance when the number of distributed processors increases.

Dimensionality: To study the effect of dimensionality on classification, we embed the original Wikipedia network (i.e., $\mathbb{R}^{4777 \times 4777}$ ) into five different dimensional spaces: $\mathbb{R}^{4777 \times 3}, \mathbb{R}^{4777 \times 16}, \mathbb{R}^{4777 \times 64}, \mathbb{R}^{4777 \times 128}$ and $\mathbb{R}^{4777 \times 256}$. The results are illustrated in TABLE 7. We can see that the performance of RTDGE increases with the dimensionality of the embedded space, and 256D embedding achieve the highest scores. On the other hand, 3-D embedding is able to achieve a decent performance with significantly reduced computation cost.

Speed Performance: Fig. 11 shows the speed up versus the number of partitions. As expected, the speed up ratio increases with the number of partitions (linear when $K$ is less than 10). 
TABLE 7

Performance evaluation w.r.t. dimensionality.

\begin{tabular}{|c|c|c|c|c|c|c|c|c|c|c|}
\hline Metric & Dimensionality & $10 \%$ & $20 \%$ & $30 \%$ & $40 \%$ & $50 \%$ & $60 \%$ & $70 \%$ & $80 \%$ & $90 \%$ \\
\hline \hline Micro-F1 & 3D & 45.70 & 46.64 & 46.74 & 46.91 & 46.96 & 47.05 & 47.09 & 47.12 & 47.23 \\
\cline { 2 - 10 } & 16D & 46.57 & 46.79 & 46.83 & 46.90 & 46.98 & 47.06 & 47.12 & 47.34 & 47.36 \\
\cline { 2 - 10 } & 64D & 46.69 & 46.83 & 46.90 & 46.91 & 46.99 & 47.08 & 47.17 & 47.40 & 47.51 \\
\cline { 2 - 10 } & 128D & 46.75 & 46.84 & 46.90 & 46.96 & 47.03 & 47.10 & 47.18 & 47.41 & 47.74 \\
\cline { 2 - 10 } & 256D & 46.78 & 46.89 & 46.95 & 46.94 & 47.05 & 47.19 & 47.38 & 47.56 & 47.75 \\
\hline \hline Macro-F1 & 3D & 62.72 & 63.59 & 63.70 & 63.85 & 63.91 & 63.95 & 64.02 & 64.05 & 64.15 \\
\cline { 2 - 10 } & 16D & 63.65 & 63.75 & 63.79 & 63.86 & 63.92 & 63.99 & 64.03 & 64.26 & 64.27 \\
\cline { 2 - 10 } & 64D & 63.71 & 63.75 & 63.79 & 63.86 & 63.93 & 64.01 & 64.05 & 64.31 & 64.38 \\
\cline { 2 - 9 } & 128D & 63.54 & 63.79 & 63.84 & 63.88 & 63.94 & 64.04 & 64.11 & 64.32 & 64.61 \\
\cline { 2 - 10 } & 256D & 63.74 & 63.84 & 63.85 & 63.89 & 63.97 & 64.12 & 64.30 & 64.46 & 64.63 \\
\hline \hline
\end{tabular}

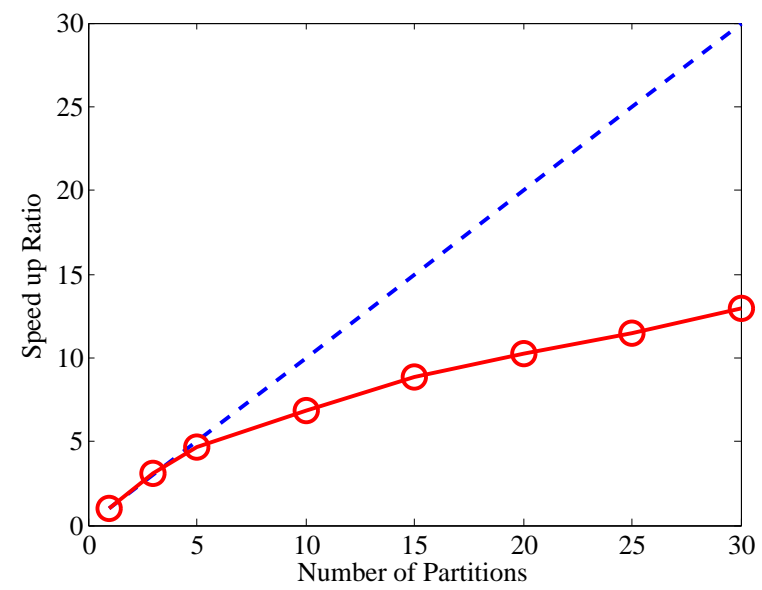

Figure 11: Speed up w.r.t Number of Partitions 


\section{CHAPTER IV}

\section{REAL-TIME DEEP ANALYSIS OF TWITTER NETWORK}

In previous chapter, the distributed graph embedding platform and the first algorithm RTDGE have been introduced. A real-world application: Twitter network analysis is demonstrated in this chapter. The Twitter data including posted twitters, following and follower lists of Twitter accounts can be retrieved in real-time. A comprehensive graph is generated to describe the relationships among Twitter users by combining those multiple data types from Twitter. Then, the proposed graph partitioning and embedding algorithm RTDGE is adopted to learn the representations of Twitter users in lower dimensional vector space. Furthermore, real-world tasks are applied including similar node detection, cluster and community classification and visualization.

Nowadays, millions people share their thoughts, stay in touch with friends, meet new people and make work-related connections on social medias. Such social networks whose nodes represent people or other entities embedded in the social context, and whose edges represent interactions, collaborations or influences between entities are highly dynamic objects. They grow and change quickly over time through the addition/deletion of edges, signifying the appearance of new interactions in the underlying social structure. Twitter network is one of the fastest growing social media network. It becomes a source of varied kind of information, and any new type of data can be harvested from it. People 
freely comment, discuss, compliment, argue and complain over topics they interested in, no matter where they come from, what religious belief they hold, rich or poor. Studies ([54-57]) have well recognized that those contents and interactions generated by Twitter users should be utilized for many applications. Collecting such rich and essential information and understanding the deep relationships of social interactions among users by which they evolves are fundamental questions that are still not well studied. Therefore, this forms the motivation for our work.

Most of Twitter analysis approaches only focus on the semantic analysis of tweets, i.e., instant messages created and spread by Twitter users or one simple relationship between pair of Twitter users, i.e., if they are friends in Twitter network. Semantic analysis of tweets is capable of extracting useful information from tweets, but the overall or general tendency towards topics and structural roles of people can rarely be captured due to their presentations in the diverse scenarios. For example, people read news about elections, it is expected to get an overview about the support and opposition for presidential candidates from Twitter. Fans of sports are fascinated about what is going on on the pitch and the reactions from other people. In all of these scenarios, a comprehensive analysis of the topics and interactions is needed. Twitter topic plays an important role in such scenarios. People are connected to community-driven content which has a certain topic, when they simply add a hash symbol \# in the tweets, known as topic of Twitter. It also benefits people to categorize and emphasize their options in their tweets by using topics hashtags. The statistics shows that among 0.5 million randomly selected tweets, around $35 \%$ of them have at lease one topic involved. In the Twitter network, it also shows a great potential that people more likely tweet similar content and 
follow each others, if they involve in same topics. Besides the relationship between user and topic, it is also important to dig the connection between topics. In the experiment, the probability for any two co-occurring topics to share same sentiment polarity is observed as over 0.8055 [58].

However, none of existing approaches is capable of real-time extraction and analysis of Twitter network. Furthermore, none of them jointly consider multiple relationships among the Twitter users. As a result, we propose a unique information extraction framework which is able to create a real-world complex graph from Twitter network by maximally retrieving and combining structural information and social interactions in real-time. The first implementation of the framework also is built with consideration of three types of relationship graphs: (1) friend relationships among users; (2) topic involving relationship between user and tweet; (3) Twitter topics co-occurrence relationship.

Once the graph is ready, a powerful social network analysis tool is desired. The traditional social network analysis approaches, e.g., PageRank [59], matrix factorization $[20,23,24]$, are unable to deal with large-scale networks, since they cannot avoid the iterative matrix calculation or the computationally expensive eigen-decomposition. Recent graph embedding approaches $([16,25-27])$ which are inspired by the advancement in natural language processing embed each node of the graph into a lower dimensional space while preserving the maximal structural information. The vectors is proved to facilitate future applications such as link prediction or node classification. While graph embedding is an intriguing idea, the existing algorithms have two major limitations: (1) None of them can perform real-time graph embedding of streaming data. Specifically, current graph 
embedding algorithms rely on prior knowledge of the entire graph and can only process the data in a batched fashion, which is not applicable to real-time streaming applications. (2) Most graph embedding algorithms are centralized, which is unable to handle big data. Twitter generates massive graph data (e.g., interactions) in a very short period of time. In this case, even a super computer could quickly deplete its resources (i.e., computation, memory and storage). A distributed graph embedding platform based Apache storm has been implemented which embeds the complex graph into a lower vector space.

Consequently, we propose a novel approach that generates a complex Twitter network by combining such multiple relationships and interactions, and analyses the similarity roles of Twitter users by using a distributed graph embedding method which is able to facilitate any future social network based tasks. Our contributions can be summarized as follows: (1) A framework is proposed to retrieve multi-types data by applying Twitter API in real-time; (2) the first implementation of the framework is built which dynamically generates a complex relation graph of certain Twitter users; (3) distributed graph embedding algorithm is adopt to our platform for real-time graph embedding.

\section{A Real-time Complex Twitter Network Extraction}

We propose a frame work in this section to jointly model the topological structure and social activity information. We also provide an first comprehensive implementation of our frame work. A complex Twitter network, denoted as $G^{*}\left(V^{*}, E^{*}\right)$, is created by combining multiple fundamental graphs in real-time. The link and its strength in the complex graph between any pair of users reflects the social activity information. 


\section{Fundamental Graphs}

To accomplish the goal of real-time retrieving data from Twitter, Twitter API has been developed. Twitter provides two different types of APIs: (i) RESTful API and (ii) Streaming API. RESTful is used for historical Twitter data by setting up certain time duration. On the contrary, Streaming API is able to monitor and retrieve data in real-time. We successively implement our method by using both types of Twitter APIs. Additionally, Twitter builds four libraries regarding to different coding languages, The library we use is twitter4j. Instead of using text-based Twitter data which could be heterogeneous and computationally expensive, we focus on combining multiple relationships from the Twitter API. Specifically, in this paper, we extract three different fundamental graphs to optimally represent the topological structure of the Twitter network and the social activity information. Note that any other extracted graphs can be added to our frame work.

Definition 1 User Following Graph: $G_{1}$ Intuitively, the following or followers in Twitter defines the most basic relationship between pair of nodes. Assume $G_{1}=\left(V_{1}, E_{1}\right)$ denotes the user following graph in which each node $n_{i} \in G_{1}$ is a Twitter user and the edge $e_{i j}$ between users $n_{i}$ and $n_{j}$ represents if they followed each other or not.

Definition 2 Topic Involving Graph: $G_{2}$ This graph describes the relation between Twitter topics and Twitter users. There two two different types of nodes in this graph: (i) Twitter user, e.g., realDonaldTrump and (ii) topics e.g., hashtag \#PRStrong. Each edge connects between a user node and a topic node, and it represents the number of times that one users has tweeted about the topic.

Definition 3 Topic Co-occurrence Graph: $G_{3}$ This graph is aiming to define the relationship among topics. Hence, the graph consists of topic nodes only. An edge exists, 
if two different topics are appeared in same tweet. For instance, the Twitter user realDonaldTrump uses \#NYCStrong\#USA in one of his tweets. Then, there is an edge between \#NYCStrong and \#USA. The weight of an edge is the number of times of the co-occurrence of two topics.

\section{Complex Graph Generation}

We now introduce how to generate a complex graph which represents the topological structure and social activity information from Twitter network. Assume the complex graph is generated by:

$$
G^{*}\left(V^{*}, E^{*}\right)=\operatorname{gen}\left(G_{1}, G_{2}, \ldots, G_{L} \mid \text { policy }\right)
$$

where $G_{1}, \ldots, G_{L}$ is the number of $L$ fundamental graphs can be retrieved by Twitter API, and the policy is the designed generation rule.

Definition 4 policy (Complex Graph Generation Rule) It defines how to combine multiple graphs into one complex graph. More specifically, the policy is given to find the feasible mappings from each fundamental graph to the complex graph. Therefore, the intensity, i.e., $\omega_{i j}^{*}$ of the relationship between pair of nodes, i.e., $e_{i j}^{*}$ in the complex graph $G^{*}$ can be computed once a policy is defined.

In this paper, we build the first implementation of this frame by using a simple linear combination method, such that the complex graph is given by:

$$
G^{*}=\lambda_{1} G_{1}+\lambda_{2} G_{2}+\ldots+\lambda_{L} G_{L}
$$

where $\lambda_{1}+\lambda_{2}+\ldots+\lambda_{L}=1$. We consider the three fundamental graphs discussed in 
Section 1. Each of simple graph has partial effort to impact the relationship of nodes, such as if two users join more common topics, or if the topic which they involved are strongly related, then they should be closer.

The first element in Eq. 29 is aiming to reflect the user following relationship by multiplying the weight coefficient parameter $\lambda_{1}$ with the edge $e_{i j}^{(1)}$ in graph $G_{1}$, i.e., the user following graph. Note that, $e_{i j}^{(l)}$ to indicate that if there is an edge existing between node $i$ and node $j$ in fundamental graph $G_{l}$ or not. More over, the weight of the edge is denoted as: $\omega_{i j}^{(l)}$. When $\omega_{i j}^{(l)}>0$, the edge $e_{i j}^{(1)}$ exists in graph $G_{l}$. Furthermore, the second item in Eq. 29 is to accomplish the goal of compromising topic involving relationship between user $i$ and user $j$, we assume Topic $_{i}$ indicates the topic set that user $i$ has involved, similarly, we have Topic $_{j}$ to be the topic set for user $j$. So that, if both user $i$ and user $j$ join the topic $h$ (i.e., they have tweeted at least one tweet with regarding to topic $h$ ), we are able to find the corresponding edges $e_{i h}^{(2)}$ and $e_{j h}^{(2)}$ in fundamental graph $G_{2}$, i.e., topic involving graph, and the corresponding weights are $\omega_{i h}^{(2)}$ and $\omega_{j h}^{(2)}$, respectively. It is reasonable to assume that if two users tweet more frequently with respect to a same topic, their Twitter activities are more in common. Noticing that we do not try to distinguish the users attitude to the topic (i.e., positive or negative) yet, we only focus on the degree of the involvement between user and the topic. Therefore, the compromised intensity of the relationship in terms of topic $h$ between user $i$ and user $j$ can be expressed as: $\lambda_{2} *\left(\omega_{i h}^{(2)}+\omega_{j h}^{(2)}\right)$. Consequentially, for all shared topic $h$, i.e., $\forall h \in T o p i c_{i} \cap T o p i c_{j}$, we use the sum of the weights and times with weight coefficient $\lambda_{2}$ to represent the impact of the relationship between $i$ and $j$ from graph $G_{2}$. The last but not least, we consider the relationship from $G_{3}$ by checking the relationships among the uncommon topics. For instance, topic $k \in T o p i c_{i}$ and topic 


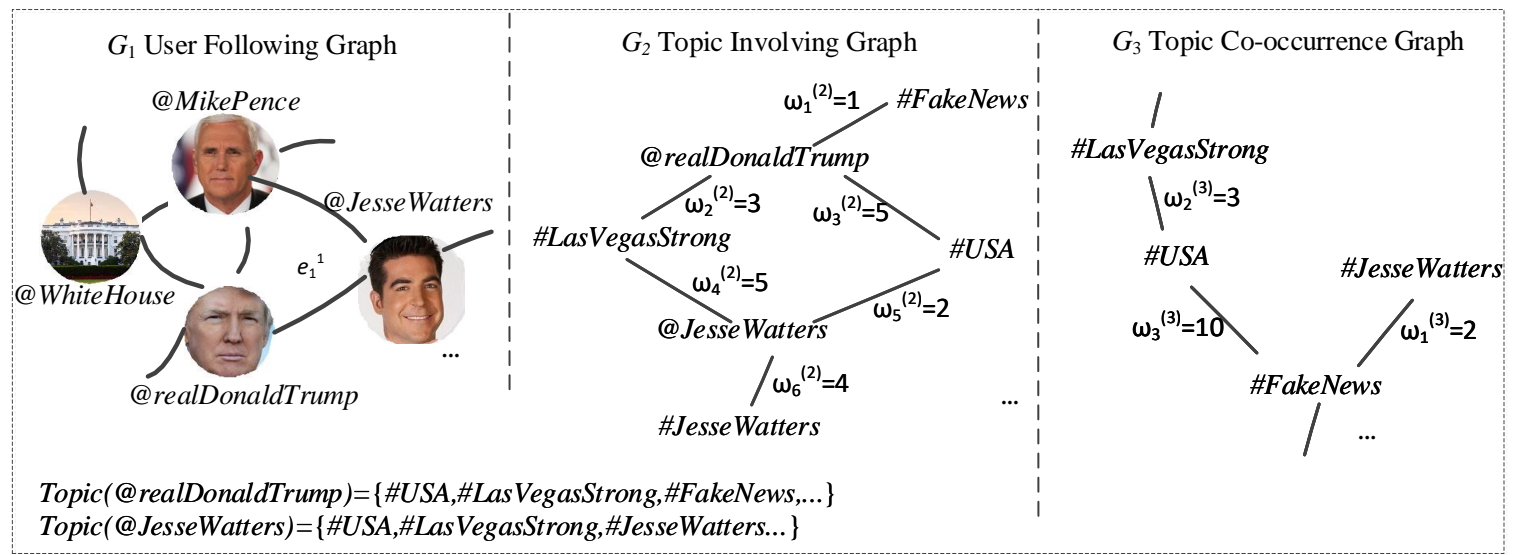

(a) Three fundamental graphs

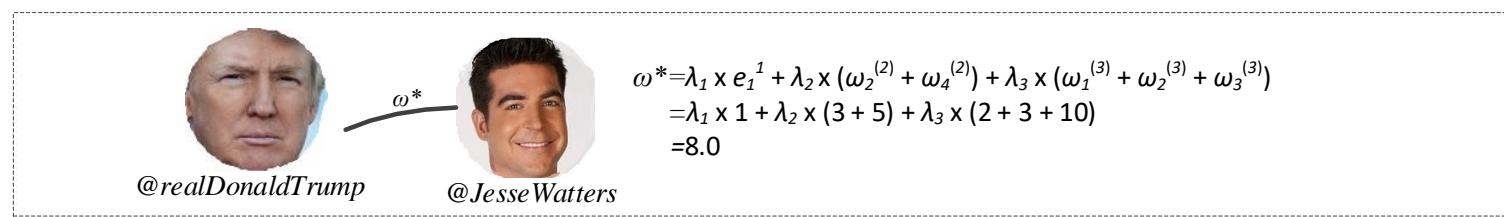

(b) An edge in the complex graph

Figure 12: An example of the complex graph generation.

$p \in$ Topic $_{j}$, and if $\omega_{k p}^{(3)}>0$ (i.e., topic $k$ and topic $p$ used to appear in at least one tweet together), we compromise such relationship by weighted with coefficient $\omega_{3}$ in the essential intensity between user $i$ and user $j$.

Hence, the edge set $E^{*}$ of the complex graph $G^{*}$ by combing fundamental graph $G_{1}, G_{2}$ and $G_{3}$ can be written as:

$$
E^{*}=\left\{\forall e_{i j} \mid \omega_{i j}^{*} \neq 0\right\}
$$

where $\omega_{i j}^{*}=\lambda_{1} * e_{i j}^{(1)}+\lambda_{2} *\left(\sum_{h \in \text { Topic }_{i} \cap \text { Topic }_{j}} \omega_{i h}^{(2)}+\omega_{j h}^{(2)}\right)+\lambda_{3} * \sum_{h, k \in \text { Topic }_{i} \cup \text { Topic }_{j}} \omega_{j h}^{(3)}$.

We demonstrate an example in Fig.12. As we can see from Fig.12(a), realDonaldTrump and JesseWatters are friends. Furthermore, there are two common topics they have involved: \#LasVegasStrong and \#USA. Additionally, among all the topics of theirs, the topic \#FakeNews and topic \#JesseWatters have co-occurrence relations as shown in $G_{3}$. Hence, the final weight of the edge between realDonaldTrump and 
JesseWatters in the complex graph is calculated as demonstrated in Fig.12(b).

\section{B Results}

In this section, the implementation of our system and the results are demonstrated. The experiments show that our algorithm outperforms other graph representation learning approaches, and more importantly, it is able to process real-time Twitter data and complete practical applications. The experiments include two parts: (i) Firstly, the analysis of real-time and real-world Twitter network is completed by implementing our real-time graph embedding and analysis platform; (ii) Then we apply our approach on some classic applications, i.e., link prediction and node classification with some popular sets to verify our performance.

\section{System Setup}

In order to implement our algorithm and evaluate its real-world performance, we develop a real-time distributed graph embedding platform based on Apache Kafka and Apache Storm. The system consists of three servers: each server has 8GB memory, quad-core Intel Xeon CPU and 500GB disk space. The connection among servers are constructed by Apache Zookeeper. Unlike GraphX that is only built upon Apache Spark, in our platform the streaming data are retrieved by Apache Kafka and distributed into multiple brokers according to an advanced real-time edge partition algorithm from [60]. A typical Apache Kafka consists of a producer and a consumer. Multiple producers and consumers can publish and retrieve data at the same time. A data message is defined as a topic, which can be divided into multiple partitions. One or more partitions can be stored 
in each broker. The data in each broker can be consumed by one or multiple Apache Storm clusters including one Apache Storm Nimbus and multiple Apache Storm Supervisors.

TABLE 8

Generated complex graphs

\begin{tabular}{|c|c|c|c|}
\hline Graph Notation & $|V|$ & $|E|$ & Description \\
\hline$G_{1}$ & 267761 & 142879 & 3 level user following graph for realDonalTrump \\
\hline$G_{1}^{\prime}$ & 46 & 873 & User following graph among realDonaldTrump friends \\
\hline$G_{2}$ & 3154 & 5497 & Topic involving graph \\
\hline$G_{3}$ & 2540 & 3859 & Topic co-occurrence graph \\
\hline$G_{1}^{*}$ & 46 & 568 & $G_{1}^{*}=$ gen $\left(G_{1}, G_{2}, G_{3} \mid\right.$ policy $\left.y_{1}^{*}\right)$, policy $=\left\{\lambda_{1}=0.1, \lambda_{2}=0.8, \lambda_{3}=0.1\right\}$ \\
\hline$G_{2}^{*}$ & 46 & 568 & $G_{1}^{*}=$ gen $\left(G_{1}, G_{2}, G_{3} \mid\right.$ policy $\left.y_{2}^{*}\right)$, policy $=\left\{\lambda_{1}=0.1, \lambda_{2}=0.8, \lambda_{3}=0.1\right\}$ \\
\hline$G_{3}^{*}$ & 46 & 568 & $G_{1}^{*}=$ gen $\left(G_{1}, G_{2}, G_{3} \mid\right.$ policy $\left.y_{3}^{*}\right)$, policy $=\left\{\lambda_{1}=0.1, \lambda_{2}=0.8, \lambda_{3}=0.1\right\}$ \\
\hline$G_{1}^{* *}$ & 46 & 568 & Complex graph generated by policy $y_{1}^{* *}$ \\
\hline$G_{T H}$ & 813 & 62907 & User following graph for realDonalTrump and HillaryClinton \\
\hline$G_{T H}^{*}$ & 443 & 34966 & Complex graph by combining $G_{T H}, G_{2}$ and $G_{3}$. \\
\hline
\end{tabular}

\section{Twitter Network Analysis}

In this section, we implement the task based on real-time and real-world Twitter network data. A new task named synonymic user detection has been designed to verify the capability of our system to learn the similarities and dig deep relationships among Twitter users. The goal of this task is to find the analogical user for a given Twitter user. More specifically, for a particular Twitter account, we try to locate its closest node who holds/acts similarly, e.g., share same structural pattern, interests or social activities. Furthermore, such model can be extended to friend or foe detection (FFD). Firstly, we extract data from the Twitter network, as shown in Table 8. The data is collected from Oct.22 to Nov 3rd, 2017. We choose Twitter account realDonaldTrump as our test node. The complex graphs are generated by using three different parameter settings, i.e., polic $y_{1}^{*}$, polic $y_{2}^{*}$, and policy* as illustrated in the Table 8. To find the synonymic node of realDonaldTrump, the node who 

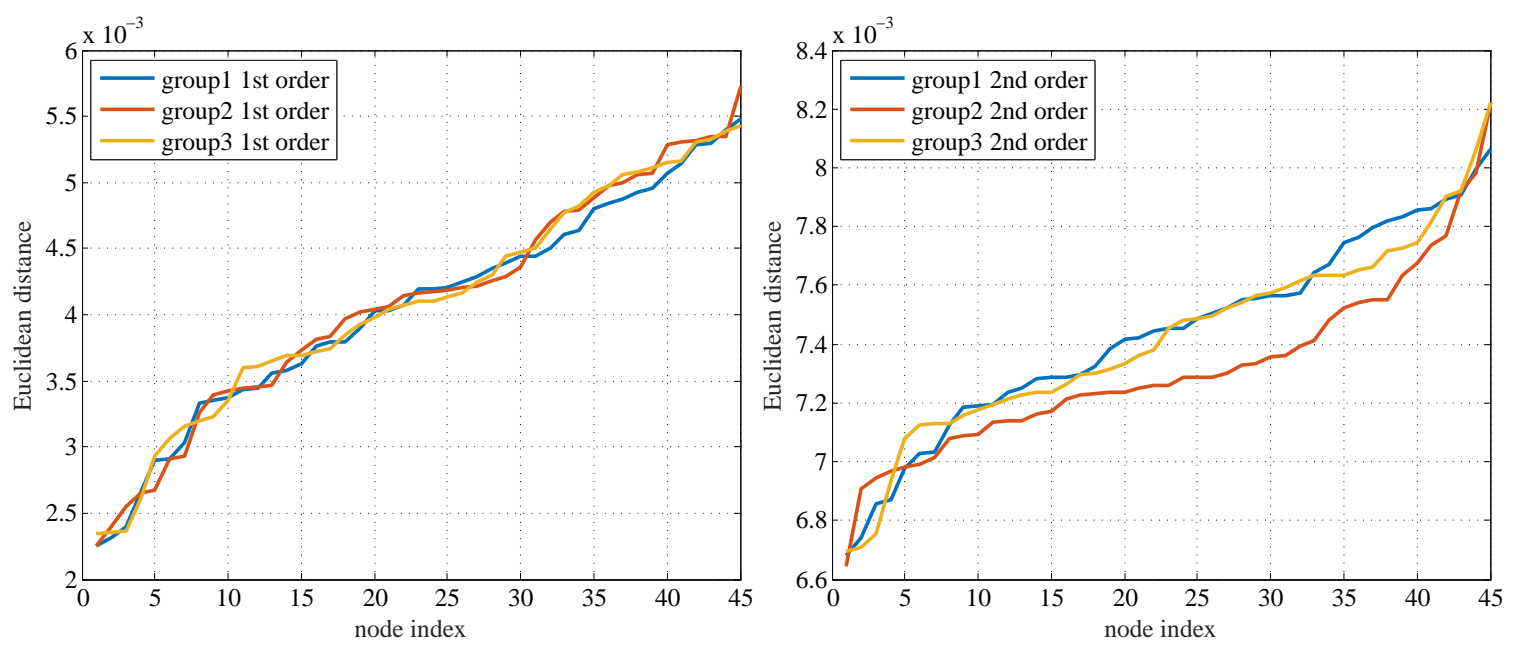

(a) Complex graph is generated by using $G_{1}, G_{2}, G_{3}$.
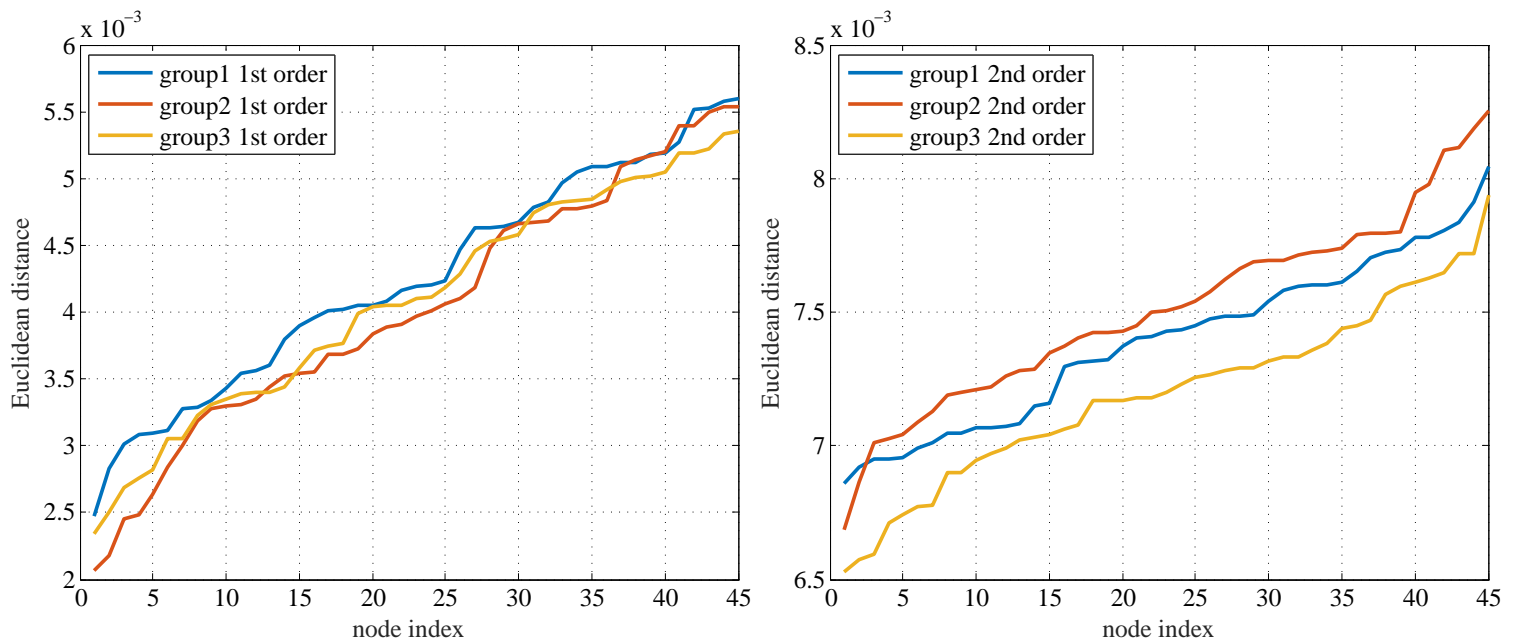

(b) Complex graph is generated by using $G_{1}^{\prime}, G_{2}, G_{3}$.

Figure 13: Distance between realDonaldTrump and his friends under policy $y_{1}^{*}$ ), policy $y_{2}^{*}$ ) and policy ${ }_{3}^{*}$ ), respectively. 
has shortest distance to node realDonaldTrump is defined as the most similar node in the network. Note that the distance can be calculated by various measurements, e.g., cosine distance, Euclidean distance. Here, we use Euclidean distance. The results regarding to the three different policy are demonstrated in Fig. 13. Y-axis is the Euclidean distance to realDonaldTrump, and the $\mathrm{X}$-axis is the node index from the closest to farthest. The more steeper slope means the nodes are more distributed under the policy. So that, the most similar node is more easier to be distinguished. Table 9 lists the top three closest nodes to realDonaldTrump under first-order proximity and second-order proximity, respectively. We find out all of them are President Trump's relations, e.g., IvankaTrump or strong supporters, e.g., jessebwatters.

TABLE 9

Closest Twitter users of realDonaldTrump

\begin{tabular}{|c|c|c|c|}
\hline \multirow{2}{*}{ Generation policy } & \multicolumn{3}{|c|}{ Top three closest Twitter users of realDonaldTrump } \\
\cline { 2 - 4 } & 1st order proximity & 2nd order proximity & 1st+2nd order proximity \\
\hline \multirow{3}{*}{ policy $y_{1}^{*} \cdot$} & 'jessebwatters' & 'Trump' & 'jessebwatters' \\
& 'Trump' & 'IvankaTrump' & 'Reince' \\
& 'LaraLeaTrump' & 'CLewandowski & 'seanhannity' \\
\hline \multirow{2}{*}{ policy $y_{2}^{*}$} & 'LaraLeaTrump' & 'TeamTrump' & 'TeamTrump' \\
& jessebwatters & 'garyplayer' & 'KellyannePolls' \\
& 'KellyannePolls' & 'DiamondandSilk' & 'LaraLeaTrump' \\
\hline \multirow{2}{*}{ policy $y_{3}^{*}$} & 'jessebwatters' & 'garyplayer' & 'IngrahamAngle' \\
& 'MrsVanessaTrump' & 'TuckerCarlson' & 'MrsVanessaTrump' \\
& 'KellyannePolls' & 'IngrahamAngle' & 'LaraLeaTrump' \\
\hline
\end{tabular}

The Table 10 shows the result when we also retrieve Twitter data for both realDonaldTrump and HillaryClinton. By applying policy, the complex graph $G_{T H}^{*}=\operatorname{gen}\left(G_{T H}, G_{2}, G_{3} \mid\right.$ policy $\left.y_{1}^{*}\right)$ is generated which contains 443 Twitter users and 34966 edges among them. Note that, each of those 443 users either has friend relationship with realDonaldTrump or HillaryClinton, or is involved in same topic(s). Among the 
closest nodes to realDonaldTrump, they have very strong Twitter activities with him. We find that most of their tweets are about realDonaldTrump's options or actives. For HillaryClinton, the closest nodes are all her supporters while they follow each others' Twitter accounts.

\section{TABLE 10}

Closest nodes detection

\begin{tabular}{|c|c|c|}
\hline Rank & Closest nodes to realDonaldTrump & Closest nodes to HillaryClinton \\
\hline 1 & 'GradyKeefe' & 'GregHale1' \\
2 & 'EmmyA2' & 'JW4Hillary' \\
3 & 'mayaharris_' & 'JessLivMo' \\
4 & 'shondarhimes' & 'GirlsWhoCode' \\
5 & 'CraigBrownNH' & 'emilieswp' \\
\hline
\end{tabular}




\section{CHAPTER V}

\section{HIERARCHICAL STRUCTURE EMBEDDING OF KNOWLEDGE}

\section{GRAPH}

In this chapter, the approach I proposed to learn the representations in a knowledge network is demonstrated. It successively captures the structural identity of entities and relations. A multi-layer hierarchical tree is constructed that measures the structural similarity between nodes pair, and applied to generate the context by random walks for a given node. Furthermore, the representations is trained for the entities and relations by a novel learning model with an advanced compositional operator. The experimental results have shown that our model successfully improve the performances of the embedded vectors in multiple tasks and on a variety of benchmark data sets, in comparison to state-of-art algorithms. Our approach overcomes their limitations by capturing explicitly the structural information of the knowledge graphs.

Our main contributions are: (i) By considering the multi-hop structure of a complex knowledge graph, a multi-layer graph is generated where each layer corresponds to the structural similarity of both entity and relation at each level of the hierarchy. (ii) Instead of only using a set of independent triples, the relationships between relations are also considered. (iii) A novel embedding method is proposed to learn the representation of entities by using an efficient and effective composition operator. 

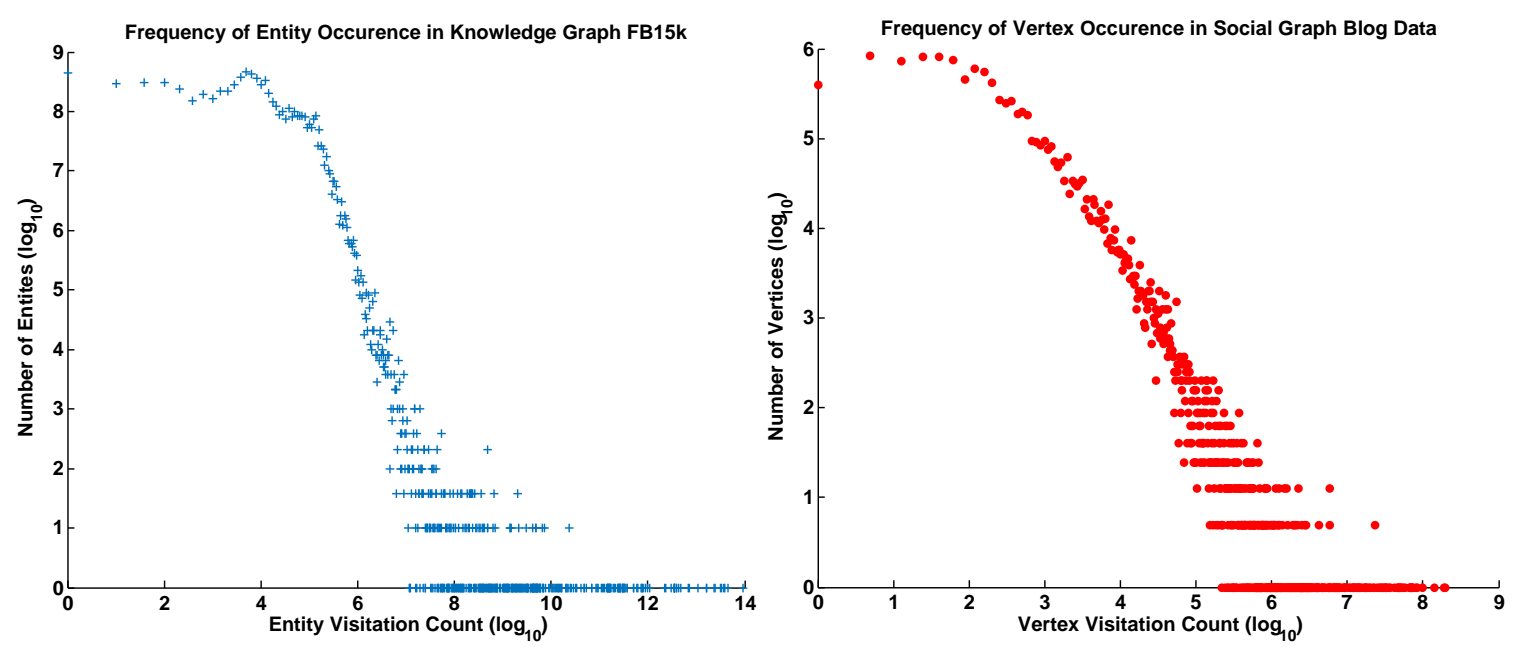

Figure 14: The distribution of entities appearing in random walks in FB15k follows a similar power-law distribution as in Blog data social network.

\section{A System Model}

\section{Scale-free Network}

Most of existing knowledge graph learning algorithms barely discuss the structure of the knowledge graph. In order to confirm the relation between classic graph and knowledge graph, we test the distribution of the knowledge graph by taking random walks. Usually, in a typical social graph, the degree of any node follows a power law distribution, i.e., scalefree network. We observe that the frequency which vertices appear in the random walks also follow a power-law distribution. The idea is to testify that if the random walks taken in a knowledge graph should also follow same distribution. Such that, the techniques from social graphs account for knowledge graphs. Figure 14 shows two distributions. The left figure is the entities occurrence in random walks which taken in knowledge graph FB15k, and the right one comes from random walks among social media graph: BlogCatalog which is a network of social friendship relations of the bloggers publicly available on BlogCatalog web site (http://blogcatalog.com). The result proves that a typical knowledge graph can be 
treated as a scale-free network.

\section{Mathematical Definitions}

For a knowledge graph, we follow the classic definition that it consists of triples. A triple is denoted as $(h, r, t)$, where $h$ is the head entity, $t$ is the tail entity, and $r$ is the relation connecting these two entities. The representation learning of the knowledge graph is conducted by the triples in aforementioned translate-based approaches. In the translate model (i.e., $|h+r| \approx t$ ), all the original triples are positive samples, and those triples constructed from the original graph are called negative samples. The latent representations of entities and relations in a lower vector space are learned by simultaneously maximizing the likelihood of positive samples and minimizing the likelihood of negative samples. While this model is simple and effective, it cannot capture the deep structure of the graph since the triple set and the sampling method cannot explore multiple-hop relations. In order to capture the structural identities of nodes in a graph, the embedded representations of nodes should be strongly correlated to their structural similarities. More specifically, since knowledge graphs have multi-type edges, the structural identity considers the impacts from both the connections between entities and relationships between relations.

The skeleton graph of a knowledge network is denoted as $G=(V, E)$, where vertex set $V$ contains nodes representing head and tail entities, and an edge $e=(u, v) \in E$ represents a relation between node $u$ and $v$. The structural similarity between two nodes $u, v$ is denoted as $f(u, v)$. Moreover, the relation similarity between two nodes $u, v$ is defined as $l(u, v)$. 


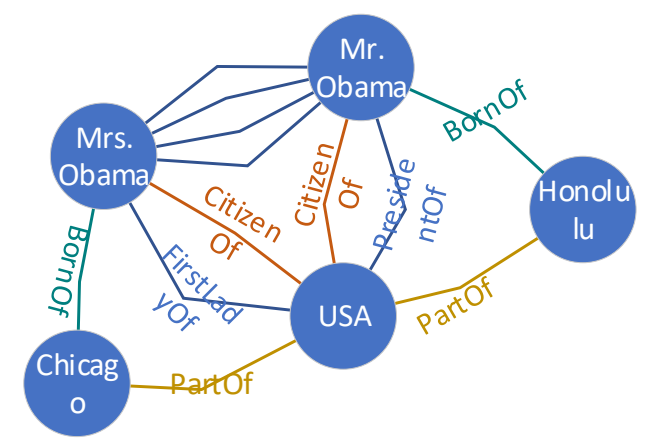

Figure 15: Knowledge Graph.

\section{Structural Distance Calculation}

In a knowledge graph, without considering edge and node attributes, the structural identity of a node is measured by its connections such as degrees (the number of direct neighbors), weights of edges, and multi-hop neighborhoods. Apparently, two nodes with the same degree have some structural similarity, but it only describes the local (single-hop) connection pattern. In this paper, we propose a new approach to capture the hierarchical structure of the knowledge graph. More specifically, the similarity between any pair of entities is measured accumulatively from their first hop neighbors to their $k$-th hop neighbors. By considering up to $k$-hop neighbors, the structural distance between two nodes $(u, v)$ is defined as:

$$
\begin{gathered}
f_{k}(u, v)=f_{k-1}(u, v)+g_{1}\left(s\left(N_{k}(u)\right), s\left(N_{k}(v)\right)\right)+ \\
g_{2}\left(N_{k}(u), N_{k}(v)\right)
\end{gathered}
$$

where $s($.$) represents the ordered degree sequence of a node set S$, and $g_{1}(\cdot, \cdot)$ measures the distance between the two integer sequences. The first term is the hierarchy of structural similarity. The goal of the second term is to measure the difference of the k-hop neighborhood sets. The ordered degree sequence $s(\cdot)$ is used to describe the degree of 
each node in the set. Figure. 15 shows partial of FreeBase knowledge graph. With respect to the node $u=M r$. Obama, we know its 1-hop neighbor set $N_{1}(u)=\{$ Mrs.Obama, Honolulu, USA $\}$, and $N_{2}(u)=\{$ Chicago $\}$. Hence, we can have $s\left(N_{1}(u)\right)=[2,6,7]$. The last term in (2) is used to measure the differences of the $k$-hop neighborhood set in order to distinguish the difference when they have identical degree sequences.

We adopt the Dynamic Time Warping method to calculate the distance between two integer sequences. Commonly, DTW $g(A, B)$ finds the optimal alignment between two numerical sequences $A$ and $B$. Given a distance function $d(a, b)$ for the elements of the sequences, DTW matches each element $a \in A$ to $b \in B$, such that the sum of the distances between matched elements is minimized. Since for our case, the elements of the sequences $s(\cdot)$ are degrees of nodes, we are using the following distance function:

$$
d(a, b)=\frac{\max (a, b)-\min (a, b)}{\min (a, b)}
$$

Note that when $a=b$, we have $d(a, b)=0$ instead of 1 . Therefore, the calculated DTW distance between two identical sequences is zero. However, even if the entity Mrs.Obabma and Mr.Obama have the same degree sequence, we cannot claim they have identical structure since they have different neighbor nodes as illustrated in Figure.15. As a result, $g_{2}\left(N_{k}(u), N_{k}(v)\right)$ is applied to reflect such differences. We have:

$$
g_{2}\left(N_{k}(u), N_{k}(v)\right)=\log \left(\left|\overline{N_{k}(u) \cap N_{k}(v)}\right|\right)
$$

which indicates the number of different $k$-hop neighbor nodes. Note that $f_{k}$ accumulatively records the hierarchy structure differences between node $u$ and $v$ which means it is nondecreasing. Furthermore, we know that if the k-th hop neighbors of $u$ and $v$ are isomorphic, 
then $f_{k}(u, v)=0$.

\section{Relation Similarity Calculation}

Similarly to the structural similarity calculation, the impact of relation type is also considered. In previous work, the relations are categorized into four types in term of number of entities: 1-to-1, 1-to-N, N-to-1 and N-to-N. This classification of the relation is not sufficient since intuitively it only describes the local connection, i.e., directly links. What is more, it ignores the relationships between relations. Therefore, our goal is to preserve global relationships between relations and to distinguish the impact power of different relations to a same node.

In Figure.15, the relation PresidentOf has more impact power than the relation CitizenOf to define the role of entity Mr.Obama. Let $R(u)$ denote the relation set which are linked to entity $u$. Besides, $R^{+}(u)$ indicates the relations where entity $u$ acts as head, and $R^{-}(u)$ contains the relations where $u$ is the tail entity. $R_{k}(u)$ is the relation set of $N$-th order neighborhoods of node $u$. Move over, the impact power of a relation $r$ can be denoted as $\eta_{r}$. Such an impact power is evaluated by the number of entities it connected. Relations that are unique to a few entities are weighted more that commonly occurring relations. The weighting scheme we apply is the inverse log frequency of the relation occurrence. Therefore, the values of impact power from a relation $r$ on its head entity (denoted as $\eta_{r}^{+}$) and tail entity (denoted as $\eta_{r}^{-}$) are given as:

$$
\begin{aligned}
& \eta_{r}^{+}=\frac{1}{\log \left(\left|r^{+}\right|+1\right)}, \\
& \eta_{r}^{-}=\frac{1}{\log \left(\left|r^{-}\right|+1\right)},
\end{aligned}
$$




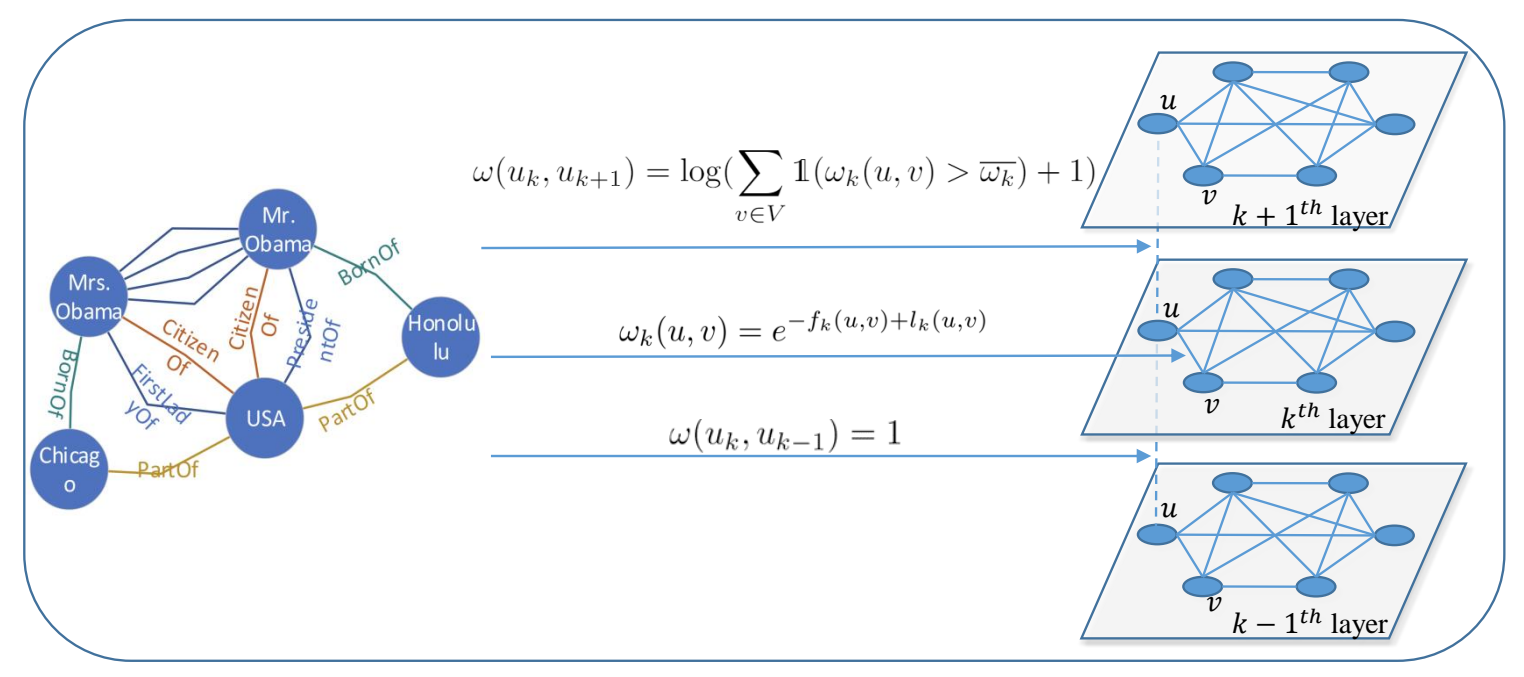

Figure 16: Construction of Hierarchy Graph $M$.

where $\left|r^{+}\right|$denotes the number of entities act as head in relation $r$. Similarly, $\left|r^{-}\right|$is the number of tail entities of relation $r$.

Relation similarity between entities are important to reflect and distinguish the similar roles that entities are treated as. The relation similarity between pair of entities is defined by the number of relations they shared, and the number of same position they located, i.e., head or tail in a triple. Then the relation similarity between any two entities $u$ and $v$ is defined as:

$$
l_{k}(u, v)=l_{k-1}(u, v)+\frac{\sum_{r \in R_{k}^{+}(u, v)} \eta_{r}^{+}-\sum_{r \in R_{k}^{-}(u, v)} \eta_{r}^{-}}{\sum_{r \in R_{k}(u, v)} \eta_{r}},
$$

where $R_{k}(u, v)=R_{k}(u) \cup R_{k}(v)$ indicates the overlapped $k$-hop relation set of entity $u$ and $v$. Note that other methods that measures the impact factor can also be applied.

\section{Structural Graph Creation}

We construct a multi-layer weighted graph that encodes the structure and relation similarities between nodes. Recall that skeleton graph $G=(V, E)$ denotes the extracted 
graph and $k^{*}$ is the diameter. Let $M$ denote the multi-layer graph with the maximal layer $k^{*}$. Each layer is formed by a weighted undirected graph with the node set $V$. The edge weight between two nodes in a layer is defined as:

$$
\omega_{k}(u, v)=e^{-f_{k}(u, v)+l_{k}(u, v)}, \quad k=0,1, \ldots, k^{*}
$$

Note that the weight is inversely proportional to structural similarity and proportional to relation similarity we calculated in previous sections.

Then the layers are connected via corresponding entities. The weight of a link between entity $u$ in level $k$ and in level $k+1$ is calculated as:

$$
\omega\left(u_{k}, u_{k+1}\right)=\log \left(\Gamma_{k}(u)+1\right), \quad k=0,1, \ldots, k^{*}-1,
$$

where $\Gamma_{k}(u)$ is the number of edges connected to $u$ whose weights are greater than the average edge weight in layer $k$ of the multi-layer graph $M$. More specifically:

$$
\Gamma_{k}(u)=\sum_{v \in V} \mathbb{1}\left(\omega_{k}(u, v)>\overline{\omega_{k}}\right)
$$

Thus, $\Gamma$ measures the similarity of node $u$ to other nodes in layer $k$. Since there are less similar nodes, when moving up to a higher layer in the content graph $M$, the context generated from $M$ for a given entity benefits the embedded representation. The weight of link between entity $u$ in layer $k$ and in layer $k+1$ is defined as:

$$
\omega\left(u_{k}, u_{k-1}\right)=1, \quad k=1,2, \ldots, k^{*} .
$$

A complexity reduction method is introduced in section 8 .

\section{Knowledge Path Generation}

The interactions of the knowledge graph (i.e., relation similarity) and the structural similarity of entities can be captured by having the hierarchy graph $M$. It is capable to 
generate rich context via taking random walks on the multi-layer graph $M$ based on the weights. Specifically, a walk steps from entity $u$ to entity $v$ in the layer $k$ is based on a probability $\operatorname{Pr}_{k}(u, v)$ which is defined as:

$$
\operatorname{Pr}_{k}(u, v)=\frac{\omega_{k}(u, v)}{\sum_{v \in V} \omega_{k}(u, v)}
$$

Note that such random walk strategy likely prefers to walk onto nodes with higher weight $\omega$. Therefore, the context of node is constructed by more structurally similar nodes which contains more information of node $u$. Intuitively, the diversity of the context would benefit the representation learning, and more importantly, we have to avoid containing duplicate nodes in the walk (otherwise it includes circles). As a result, the random walk changes to its corresponding node either in layer $k+1$ or layer $k-1$ according to the following probabilities:

$$
\begin{aligned}
& \operatorname{Pr}_{k}\left(u_{k}, u_{(k+1)}\right)=\frac{\omega\left(u_{k}, u_{k+1}\right)}{\omega\left(u_{k}, u_{k+1}\right)+\omega\left(u_{k}, u_{k-1}\right)}, \\
& \operatorname{Pr}_{k}\left(u_{k}, u_{(k-1)}\right)=\frac{\omega\left(u_{k}, u_{k-1}\right)}{\omega\left(u_{k}, u_{k+1}\right)+\omega\left(u_{k}, u_{k-1}\right)},
\end{aligned}
$$

where $\omega\left(u_{k}, u_{k+1}\right)$ and $\omega\left(u_{k}, u_{k-1}\right)$ have been given in Eq. 37 and Eq. 39.

\section{Latent Representation Learning}

In this section, we introduce a compositional representation learning model for knowledge graphs which is inspired by the skip-gram model from the nature language process (NLP) field. The basic idea of the skip-gram model is to maximize the probability of a word that appeared when given its certain context. For the knowledge graph, we treat the pair of entities i.e., triple, as a word. A critical point is how to represent the triple in vector space by building a operator $\theta_{r}(h, t)$. An existing compositional operator is tensor 
product which is denoted as $\vec{r}^{T} \cdot(\vec{h} \circ \vec{t})$. Here, we adopt a compositional operator from Plate [61] and Nickel at. al[44] that $\theta_{r}(h, t)=\vec{r}^{T}(\vec{h} * \vec{t}) . \vec{h} * \vec{t}$ is the circular correlation given by:

$$
[a * b]_{i}=\sum_{j=1}^{d} a_{j} b_{(i+1) \bmod } \quad d
$$

where $d$ is the dimensionality of the latent representation vector. Then the learning model can be written as:

$$
\operatorname{Pr}\left(\theta_{r}(h, t) \mid \Theta\right)=\frac{e^{\left(\vec{r}^{T}\left(\eta_{r}(h) \vec{h} * \eta_{r}(t) \vec{t}\right)\right.}}{\sum_{\left(h^{\prime}, r^{\prime}, t^{\prime}\right) \in \Theta} e^{\left(\vec{r}^{\prime}{ }^{T}\left(\eta_{r^{\prime}}\left(h^{\prime}\right) \vec{h}^{\prime} * \eta_{r^{\prime}}\left(t^{\prime}\right) \vec{t}^{\prime}\right)\right)}}
$$

where $\eta_{r}$ is the impactor factor for the relation $r$, and the $\Theta$ represents the context. In this paper, we apply random walks to generate sequences to determine the the context of a given node. Details are discussed in Section 6.

Remark 1 Even TransE is very simple and efficient, it can only reflect the linear and local relationships between entity pairs.

Remark 2 Tensor product is a popular method of the compositional representations of triple. However, it costs large amount of memory due to its characteristic. Details are discussed in Section 8.

Remark 3 Nickel at. al[44] successfully adopts a powerful compositional operator from Plate at.al [61] for the expression of the triples in knowledge graph. However, the optimization function used in the paper is the simple sigmoid function $\sigma(x)$, ie., $\operatorname{Pr}\left(\theta_{r}(h, t) \mid \Theta\right)=\frac{1}{1-e^{-\vec{r}^{T}(\vec{h} * \vec{t})}}$, which is only able to describe the local relations of the target entity and its context.

Skip-gram representation learning model has been studied well previously. Given the linear nature of text, the notion of a neighbor can be naturally defined using a sliding 
window over consecutive words. However, knowledge graphs are not linear, and thus a richer notion of a neighborhood is needed. To resolve this issue, we propose a knowledge path generation method that samples many different neighborhoods of a given entity by using the content graph $M$. In order to learn the representations of the relations and entities in the knowledge graph, the stochastic gradient descent (SGD) is applied.

\section{Complexity and optimization}

DTW is applied to compute the distance of two integer sequences for building the content graph $M$. While classic implementation of DTW has complexity $\mathcal{O}\left(l^{2}\right)$, fast techniques have been completed witin $\mathcal{O}(l)$, where $l$ is the size of the largest sequence [? ]. Assume the maximal degree in the original KG is $d_{\max }$. Hence, the hypothesis holds that $\left|s\left(R_{k}(u)\right)\right| \leq \min \left(d_{\max }^{k}, n\right)$, for $\forall u, \forall k$. The number of edges in each layer of graph $M$ is $\frac{n(n-1)}{2}$ pairs. Therefore, the complexity to build one layer is $\mathcal{O}\left(n^{2} \min \left(d_{\text {max }}^{k}, n\right)\right)$. As a result, it takes $\mathcal{O}\left(k * n^{3}\right)$ to generate graph $M$. Complexity reduction method is discussed as follows.

Compressed sequence During the DTW process, the complexity is affected by the length of the degree sequence. Instead of keeping the original degree sequence, we only record the number of occurrences of that degree. Therefore, the compressed degree sequence is a tuple with the degree and the number of occurrences. Since many nodes in a network tend to have the same degree, in practice the compressed ordered degree sequence can be an order of magnitude smaller than the original.

Assume the sequence $A$ and $B$ have compressed sequence as $A^{\prime}$ and $B^{\prime}$, 
respectively. Then, the DTW pairwise distance can be calculated as:

$$
\operatorname{dist}(\boldsymbol{a}, \boldsymbol{b})=\left(\frac{\max \left(a_{0}, b_{0}\right)-\min \left(a_{0}, b_{0}\right)}{\min \left(a_{0}, b_{0}\right)}\right) \max \left(a_{1}, b_{1}\right)
$$

where $\boldsymbol{a}=\left(a_{0}, a_{1}\right)$ and $\boldsymbol{b}=\left(b_{0}, b_{1}\right)$ are the compressed tuples; the degrees are represented by $a_{0}$ and $b_{0}$ while the occurrences are denoted as $a_{1}$ and $b_{1}$. Since $A^{\prime}$ and $B^{\prime}$ are much shorter than $A$ and $B$, the DTW is more efficient.

TABLE 11

\section{Compositional Representation Operator}

\begin{tabular}{ccc}
\hline Operator & Memory & Runtime \\
\hline$|h+r-t|$ & $\mathcal{O}\left(n_{e} d+n_{r} d\right)$ & $\mathcal{O}\left(n_{e}+n_{r}\right)$ \\
TensorProduct & $\mathcal{O}\left(d^{2}\right)$ & $\mathcal{O}\left(d^{2}\right)$ \\
CircularCorrelation & $\mathcal{O}(d)$ & $\mathcal{O}(d \log d)$ \\
\hline
\end{tabular}

Efficiency of circular correlation In Section 7, a novel compositional knowledge graph feature learning model is proposed which adopts the circular correlation operator to combine the triple elements. Table 11 compares the runtime and memory complexity for compositional representations. Although TransE requires very few parameters and easy to train, it compromises with the modeling power which reduces the accuracy of the embedding. As we can see, compared with tensor product, the circular correlation improves the complexity both on runtime and memory storage from $\mathcal{O}\left(d^{2}\right)$ to $\mathcal{O}(d \log d)$ where $d$ is the dimensionality of the embedded representations.

\section{B Implementation and Experiments}

In this section, the experimental results are demonstrated to evaluate the performance of our model on capturing the structural similarity of knowledge networks in 
different scenarios. Our experiments are implemented on a work station which has $32 \mathrm{~GB}$ memory, 512G SSD, and 16 AMD cores.

TABLE 12

Data Sets

\begin{tabular}{cccccc}
\hline Data Set & Relation\# & Entity\# & Train\# & Valid\# & Test\# \\
\hline WN11 & 11 & 55,166 & 164,467 & 4,000 & 4,000 \\
WN18 & 18 & 40,943 & 141,442 & 5,000 & 5,000 \\
FB15k & 1,345 & 14,951 & 483,142 & 50,000 & 59,071 \\
FB38k & 607 & 37,516 & 322,696 & 8,914 & 9,954 \\
\hline
\end{tabular}

1 Implementation

For fair comparison with benchmark works, we use the same data setup as in [41].

\section{Data Sets}

As shown in Table 12, two typical knowledge graphs are used: WordNet and FreeBase. WordNet is a large lexical database of English words, where nouns, verbs, adjectives and adverbs are grouped as set of entities (AKA synsets). The links represent the conceptual-semantic and lexical relations between entities. In this paper we employ WN18 data set which contains 18 relation types. FreeBase is known as "an open shared database of the world's knowledge", which is a collaboratively edited database of cross-linked general facts. For instance, a triple: "Obama, /people/person/place_of_birth, USA" represents that Obama was born in USA. The current read-only version of FreeBase has more than 362 million facts. In this paper we use a subset known as FB15k, which is pre-processed by [38] with 592,213 triples, 14,951 entities and 1,345 relationships. It is worth noting that the setting of FreeBase is 
TABLE 13: Entity Prediction.

\begin{tabular}{|c|cc|cc|cc|cc|}
\hline Data Sets & \multicolumn{4}{|c|}{ WN18 } & \multicolumn{4}{c|}{ FB15k } \\
\hline \multirow{2}{*}{ Metric } & \multicolumn{2}{|c|}{ Mean Rank } & \multicolumn{2}{|c|}{ Hits@ 10(\%) } & Mean Rank & \multicolumn{2}{c|}{ Hits@ 10(\%) } \\
\cline { 2 - 10 } & Raw & Filter & Raw & Filter & Raw & Filter & Raw & Filter \\
\hline SE & 1,011 & 985 & 28.8 & 39.8 & 273 & 162 & 28.8 & 39.8 \\
\hline TransE & 263 & 251 & 75.4 & 89.2 & 243 & 125 & 34.9 & 47.1 \\
\hline TransR & 238 & 225 & 79.8 & 92.0 & 226 & 78 & 43.8 & 65.5 \\
\hline CTransR & 231 & 218 & 79.4 & 92.3 & 233 & 82 & 44.0 & 66.3 \\
\hline TransHR & 210 & 75 & 81.4 & 89.1 & 212 & 67 & 47.8 & 70.0 \\
\hline HSE & 204 & 66 & 86.0 & 95.7 & 192 & 56 & 57.6 & 85.1 \\
\hline
\end{tabular}

profoundly different from WordNet. While WordNet contains arbitrary entities, entities in FreeBase are restricted for a certain relation. For instance, for relation Gender, the head entity is a person's name, and the tail can only be male or female; for relation nationality, the tail entity can only be the name of one of the 188 countries.

\section{Entity Prediction}

Unlike the traditional link prediction in single relation networks, link prediction for a knowledge graph is to complete a triple $(h, r, t)$ with missing $h$ or $t$. Furthermore, instead of one best answer, this task feeds back a set of candidates from the whole entity set of the graph. The ranking is calculated by a score function $f_{r}$ introduced by [38]. We also use the setup from [46] and [38]). There are two metrics to evaluate the results: (1) mean rank of correct entities; (2) proportion of correct entities in top-10 ranked entities (Hits@10). Hence, results in lower mean ranking and higher Hits@10 are considered better performance. Since there exists corrupted entities in the knowledge graph, a filter is applied before ranking so that the performance are evaluated based on "Raw" and "Filter" 
data sets. We compare our algorithm with TransE, TransR ${ }^{1}$ and TransHR. The learning rate of the SGD is set in the range of $[0.001,0.1]$. For these benchmark algorithms, besides the optimal configurations presented in their papers, we also select the margin value $\gamma \in\{1,1.5,2,2.5,3,3.5,4,4.5,5\}$, the embedding dimensionality as $20,50,100,128$ and the mini-batch size $B \in\{20,120,480,960,4800\}$. Our experimental results are averaged over 100 trials. The evaluations are illustrated in Table 13. As we can see, our algorithm outperforms other baseline methods consistently for both WN18 and FB15 data sets. Notably, our approach has more than $20 \%$ performance improvement over TransHR for FB15k data set with Hits@10. Table 13 clearly shows that our approach has the best performance even if the knowledge graph has a large number of relationships. The reason is that we preserve more structural information by creating the hierarchy graph and walking more reasonable paths among the relation trees.

TABLE 14

Entity Prediction by Relation Types on FB38k

\begin{tabular}{|c|c|cc|cc|}
\hline \multirow{2}{*}{ Relation Type } & Metric & \multicolumn{2}{|c|}{ Mean Rank } & \multicolumn{2}{c|}{ Hits @ 10(\%) } \\
\cline { 2 - 6 } & Models & Raw & Filter & Raw & Filter \\
\hline \multirow{4}{*}{ Sole-Relation } & TransE & 514 & 159 & 51.2 & 59.3 \\
\cline { 2 - 6 } & TransR & 503 & 112 & 49.8 & 56.5 \\
\cline { 2 - 6 } & CTransR & 517 & 103 & 50.0 & 66.0 \\
\cline { 2 - 6 } & TransHR & 475 & 92 & 51.2 & 65.8 \\
\cline { 2 - 6 } & HSE & 410 & 78 & 55.6 & 69.3 \\
\hline \multirow{3}{*}{ Hyper-Relation } & TransE & 565 & 198 & 55.4 & 67.5 \\
\cline { 2 - 6 } & TransR & 568 & 196 & 54.7 & 66.2 \\
\cline { 2 - 6 } & CTransR & 569 & 188 & 60.0 & 77.1 \\
\cline { 2 - 6 } & TransHR & 561 & 185 & 59.8 & 76.8 \\
\cline { 2 - 6 } & HSE & 478 & 161 & 65.1 & 90.7 \\
\hline
\end{tabular}

Table 14 and Table 15 show the performance of entity prediction from different

\footnotetext{
${ }^{1}$ By using the source codes and the optimal parameter settings given in [38] and [39], we cannot repeat the same results.
} 
perspectives. Table 14 shows the results for FB38k data set by considering different relation categories (i.e., sole-relation Vs. hyper-relation [42]). For fair comparison, we follow the same experimental setup as in TransHR. As expected, HSE performs the best among all models for both relation types. For example, using filtered data, our model has $14.8 \%$ and 18.1\% performance gains over TransHR for mean rank and Hits@ 10 metrics respectively. Table 15 illustrates the prediction results for head and tail entities. As we can see, our algorithm has a stable performance for both the head prediction and tail prediction. The interaction information is better preserved in our model, due to the un-commutative feature of circular correlation operation.

TABLE 15

Hits@ 10(\%) on FB38k

\begin{tabular}{|c|c|c|c|c|c|}
\hline \multirow{2}{*}{ Task } & \multirow{2}{*}{ Model } & \multicolumn{2}{|c|}{ Sole-Relation } & \multicolumn{2}{c|}{ Hyper-Relation } \\
\cline { 2 - 6 } & & Raw & Filter & Raw & Filter \\
\hline \multirow{4}{*}{$\begin{array}{c}\text { Head Prediction } \\
\text { Hits @ 10(\%) }\end{array}$} & TransE & 57.1 & 63.9 & 53.3 & 65.6 \\
\cline { 2 - 6 } & TransR & 55.5 & 68.4 & 56.8 & 72.6 \\
\cline { 2 - 6 } & CTransR & 54.7 & 68.4 & 58.5 & 76.1 \\
\cline { 2 - 6 } & TransHR & 56.3 & 70.4 & 57.0 & 74.7 \\
\cline { 2 - 6 } & HSE & 60.2 & 74.1 & 62.7 & 87.1 \\
\hline \multirow{4}{*}{$\begin{array}{c}\text { Tail Prediction } \\
\text { Hits @ 10(\%) }\end{array}$} & TransE & 45.3 & 54.7 & 57.6 & 69.5 \\
\cline { 2 - 6 } & TransR & 44.1 & 57.9 & 60.9 & 75.5 \\
\cline { 2 - 6 } & CTransR & 44.9 & 57.1 & 62.0 & 78.5 \\
\cline { 2 - 6 } & TransHR & 46.2 & 61.1 & 62.7 & 78.9 \\
\cline { 2 - 6 } & HSE & 51.0 & 64.5 & 67.5 & 94.3 \\
\hline
\end{tabular}

\section{Multi-relations Prediction}

In order to testify the capability of our model under the multi-relation scenarios, i.e., $\mathrm{N}$-to-N, N-to-1 and 1-to-N relation types between a pair of entities, we examine the results of the most frequently occurring five multi-relations. Table 16 illustrates the experimental 
TABLE 16

Multi-relation Prediction.

\begin{tabular}{cccc}
\hline \multirow{2}{*}{ Relation } & \multirow{2}{*}{ Occurring Frequency } & Hits@ 10(TransE/TransR/TransH/CTransR/TransHR/HSE) $(\%)$ \\
\cline { 3 - 4 } & & Head & Tail \\
\hline /location/location/contains & 20,597 & $95.0 / 94.4 / 96.0 / 95.9 / 97.8 / 97.6$ & $28.3 / 27.3 / 30.2 / 27.0 / 45.9 / 58.1$ \\
/location/location/containedby & 20,578 & $46.4 / 47.3 / 67.5 / 71.0 / 72.5 / 74.2$ & $95.5 / 96.2 / 91.0 / 95.0 / 98.3 / 96.3$ \\
/people/person/place_lived & 14146 & $47.5 / 42.2 / 53.7 / 53.9 / 61.1 / 73.4$ & $86.6 / 86.4 / 92.0 / 94.5 / 93.8 / 91.5$ \\
/people/place_lived/location & 14,119 & $87.4 / 87.4 / 93.5 / 96.3 / 94.6 / 97.1$ & $50.3 / 46.9 / 55.7 / 60.5 / 66.2 / 78.2$ \\
/location/location/perople_born_here & 13,715 & $91.4 / 90.0 / 96.3 / 97.9 / 97.1 / 97.3$ & $57.5 / 56.0 / 61.9 / 67.0 / 73.2 / 81.4$ \\
\hline
\end{tabular}

results. As we can see from the table, our algorithm has a better accuracy compared to the baseline approaches. For the relation /location/location/contains whose appearance achieves 20,597 in total, the accuracy of $97.6 \%$ for the head prediction can be reached. Although some time TransHR has a better performance than ours, our algorithm has a better balance between the head prediction and tail prediction. More specifically, TransHR has reduced $53.1 \%$ accuracy for the tail prediction of relation /location/location/contains (45.9\%), compared to its high accuracy for the head prediction which is $97.8 \%$. Other baseline algorithms also reveal the same trend.

\section{Triple Detection}

This task is to confirm if a given triple is correct or not, based on a binary classification of each triple. Socher et al. has used this to evaluate NTN model [62]. The metric determines accuracy based on how many triples are identified correctly.

As knowledge graphs have only positive triples and classification evaluation requires negative labels, equal number of negative samples are created by corrupting the positive triples. We follow the same generating rule as in [62] that corrupts each positive triple in the selected testing set to create a corresponding negative triple to generate a total set of double testing triples. Note that, the negative sample is generated by only replacing 
the tail entity which makes the task more difficult due to the lack of obvious non-relation triples.

The decision is made by calculating a dissimilarity score for a triple $(h, r, t)$ which is given by $s c_{r}$. The triple is labeled as correct when $s c_{r}<\sigma_{r}$, otherwise it is corrupted triple. $\sigma_{r}$ is optimized by maximizing classification accuracy on the validation set. For the comparing methods, we set the learning rate among $\{0.1,0.01,0.001,0.0001\}$, the margin $\gamma$ among $\{1,1.5,2,2.5,3,3.5,4,4.5,5\}$, embedding dimensions among $\{20,50,100,128\}$ and the batch size $B$ among $\{20,120,480,960,4800\}$. For both data sets, we traverse all the training triples for 100 rounds. The evaluation results are illustrated in Table 17. From Table 17, we observe that HSE significantly outperforms other baseline methods both on WN11 data set and FB15k data set. There is a $16.8 \%$ increasing of the accuracy between our model and TransE on WN11 data set, and a 11.2\% on FB15k data set.

TABLE 17

Triple Classification

\begin{tabular}{|c|c|c|}
\hline Dodels & Wata Sets & FB15k \\
\hline TransE & 75.9 & 79.2 \\
\hline TransR & 85.9 & 83.9 \\
\hline CTransR & 85.7 & 84.5 \\
\hline TransHR & 87.5 & 85.5 \\
\hline HSE & 91.2 & 89.2 \\
\hline
\end{tabular}

\section{Scalability}

In order to illustrate its scalability, we record the average execution time for 10 independent runs on graphs with size from 100 to 1, 000,000 entities. To accomplish the goal of speeding up the training process, skip-gram model with negative sampling method 


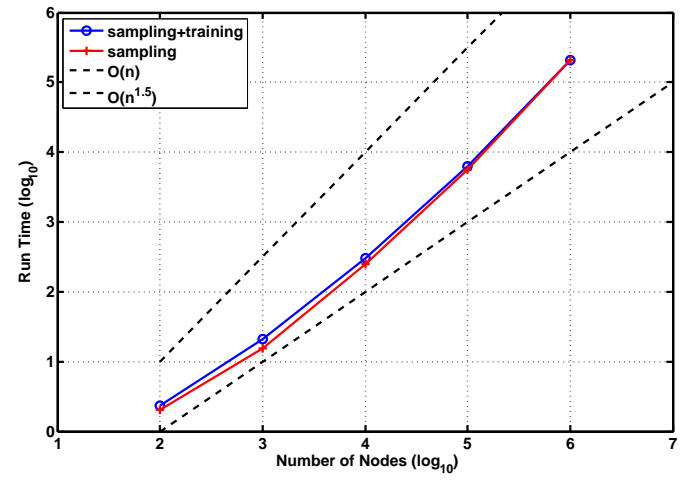

Figure 17: Scalability of Execution Time

has been adopted [63]. Figure.11 illustrates the run time indicating that our model scales close-to-linearly, compared to $n^{1.5}$ linear speed-up, i.e., the upper dash line. Therefore, despite the worst case scenario, in practice our model can be applied to large-scale knowledge networks. 


\section{CHAPTER VI}

\section{CONCLUSION AND FUTURE WORK}

\section{A Conclusion}

DSA has been extensively investigated in the last few years for CR (Cognitive Radio) networks. However, most of the DSA approaches are developed for terrestrial communications where the secondary users are fixed or moving with a low speed in a small geographic area without addressing the unique challenges in a SATCOM environment. The challenges include the error-prone spectrum sensing, the high mobility, the large GEO and LEO coverage, and a long signal delay due to long distance signal propagation. Furthermore, the LEO spectrum sensing suffers from weak GEO signals and long delay due to a long distance of the GEO signal propagation. Therefore, DSA should provide the spectrum sensing and accurate decision making under uncertainty environments for the SATCOM communications. An optimal channel access strategy increases the channel utilization while reducing the collision probability with the primary user. In this paper, we proposed a dynamic spectrum access decision-making approach to address the uncertainty in the SATCOM systems. In my approach, the optimal policies $\left\{\pi_{s}^{*}, \pi_{\delta}^{*}, \pi_{c}^{*}\right\}$ are evaluated that can maximize the overall throughput while reducing the collision probability in the high interference or jamming environment. Our simulations demonstrate the effectiveness in terms of the accumulated throughput gain. In addition, 
our approach is promising for a LEO to cognitively utilize the GEO spectrum bands. A new graph embedding framework called RTDGE has been proposed in this work, which can distributively embed large scale graph in real-time. Specifically, I proposed an edge based graph partition to ensure balanced partition. To handle streaming data input, a dynamic graph embedding approach was provided without compromising the system efficiency and effectiveness. Then, I adopted a heuristic global aggregation method to combine the locally embedded vector spaces. Finally, the RTDGE algorithm was implemented and evaluated on the planform which combined with Apache Kafka, Apache Zookeeper and Apache Storm. The experimental results on various benchmark data sets prove the effectiveness of our algorithm. A real-world application on analyzing the deep relationships among Twitter users in real-time also has been implemented.

Besides, a novel approach named hierarchical structure embedding (HSE) has been proposed to embed knowledge graphs. The algorithm is capable to learn the representations. It captures the structural identity of entities and relations in knowledge graphs. A multi-layer hierarchical graph is constructed to measure the structural similarity between entities, and applied to generate the entity's context by random walks. Furthermore, the representations is trained for the entities and relations by a novel learning model with an advanced compositional operator. The experimental results have shown that the model successfully improve the performances of the embedded vectors in multiple tasks and on a variety of benchmark data sets, in comparison to state-of-art algorithms. Our approach overcomes their limitations by capturing explicitly the structural information of the knowledge graphs. 


\section{B Future Work}

Research on graph embedding has drawn a lot of attentions these years, due to its wide usage in real-world scenarios. More and more companies are trying to gain the ability to analyze and learn the structural and hidden information from their massive graph data sets (especially for real-time applications). Our previous work successively proposed such a graph embedding framework for both single-relation and multi-relation graphs. However, there are some remaining challenges to be addressed by future work:

- Additional Proximity: All existing works apply the first-order proximity or the second-order proximity. However, the two proximities cannot be conducted at the same time. Therefore, our future work will study a third proximity which combines the first-order proximity and second-order proximity.

- Detecting the Change of Graph Topology: Our current work is able to detect the effects caused by incoming vertices or edges in the graph. In future, we aim to dig into the topology variances of dynamic graphs.

- Advanced Global Aggregation Approach: The beauty of our design is that the graph embedding results are numerical vectors, which means we can apply any supervised or un-supervised machine learning algorithms with reasonable complexity to aggregate the local results. In order to balance the computational complexity and accuracy, we plan to design two different aggregation algorithms for our framework. One is unsupervised global aggregation which has been designed in our previous work. The other approach is to apply a learning process for the global aggregation using the historical graph embedded data. 


\section{REFERENCES}

[1] C. Xin, M. Song, L. Ma, G. Hsieh, and C. C. Shen, "An incentivized cooperative architecture for dynamic spectrum access networks," IEEE Transactions on Wireless Communications, vol. 12, pp. 5154-5161, October 2013.

[2] J. Backens, C. Xin, M. Song, and C. Chen, "Dsca: Dynamic spectrum co-access between the primary users and the secondary users," IEEE Transactions on Vehicular Technology, vol. 64, pp. 668-676, Feb 2015.

[3] Q. Zhao, L. Tong, A. Swami, and Y. Chen, "Decentralized cognitive mac for opportunistic spectrum access in ad hoc networks: A pomdp framework," IEEE Journal on Selected Areas in Communications, vol. 25, pp. 589-600, April 2007.

[4] S. Kandeepan, L. D. Nardis, M. G. D. Benedetto, A. Guidotti, and G. E. Corazza, "Cognitive satellite terrestrial radios," in 2010 IEEE Global Telecommunications Conference GLOBECOM 2010, pp. 1-6, Dec 2010.

[5] A. Sahai, N. Hoven, and R. Tandra, "Some fundamental limits on cognitive radio," in in Forty-second Allerton Conference on Communication, Control, and Computing, 2004.

[6] Y. Chen, Q. Zhao, and A. Swami, "Joint design and separation principle for opportunistic spectrum access in the presence of sensing errors," IEEE Transactions on Information Theory, vol. 54, pp. 2053-2071, May 2008.

[7] G. Yang and W. Yiming, "Multi-channel access algorithm with channel state information unknown," in 2012 Fifth International Conference on Intelligent Computation Technology and Automation, pp. 427-430, Jan 2012.

[8] Y. Yilmaz, Z. Guo, and X. Wang, "Sequential joint spectrum sensing and channel estimation for dynamic spectrum access," IEEE Journal on Selected Areas in Communications, vol. 32, pp. 2000-2012, November 2014.

[9] K. Liu, Q. Zhao, and Y. Chen, "Distributed sensing and access in cognitive radio networks," in 2008 IEEE 10th International Symposium on Spread Spectrum Techniques and Applications, pp. 28-31, Aug 2008.

[10] S. Sahu, A. Mhedhbi, S. Salihoglu, J. Lin, and M. T. Özsu, "The ubiquity of large graphs and surprising challenges of graph processing: A user survey," CoRR, vol. abs/1709.03188, 2017.

[11] P. Goyal and E. Ferrara, "Graph embedding techniques, applications, and performance: A survey,” CoRR, vol. abs/1705.02801, 2017. 
[12] G. Hinton and Y. Bengio, "Visualizing data using t-sne," in Cost-sensitive Machine Learning for Information Retrieval 33, 2008.

[13] G. Hinton and S. Roweis, "Stochastic neighbor embedding," in Advances in Neural Information Processing Systems 15, pp. 833-840, MIT Press, 2002.

[14] T. Mikolov, I. Sutskever, K. Chen, G. S. Corrado, and J. Dean, "Distributed representations of words and phrases and their compositionality," in Advances in Neural Information Processing Systems 26 (C. J. C. Burges, L. Bottou, M. Welling, Z. Ghahramani, and K. Q. Weinberger, eds.), pp. 3111-3119, Curran Associates, Inc., 2013.

[15] T. Mikolov, K. Chen, G. Corrado, and J. Dean, "Efficient estimation of word representations in vector space," CoRR, vol. abs/1301.3781, 2013.

[16] B. Perozzi, R. Al-Rfou, and S. Skiena, "Deepwalk: Online learning of social representations," CoRR, vol. abs/1403.6652, 2014.

[17] J. Dean and S. Ghemawat, "Mapreduce: Simplified data processing on large clusters," Commun. ACM, vol. 51, pp. 107-113, Jan. 2008.

[18] G. Malewicz, M. H. Austern, A. J. Bik, J. C. Dehnert, I. Horn, N. Leiser, and G. Czajkowski, "Pregel: A system for large-scale graph processing," in Proceedings of the 2010 ACM SIGMOD International Conference on Management of Data, SIGMOD '10, (New York, NY, USA), pp. 135-146, ACM, 2010.

[19] S. Sakr, F. M. Orakzai, I. Abdelaziz, and Z. Khayyat, Large-Scale Graph Processing Using Apache Giraph. Springer Publishing Company, Incorporated, 1st ed., 2017.

[20] A. Ahmed, N. Shervashidze, S. Narayanamurthy, V. Josifovski, and A. J. Smola, "Distributed large-scale natural graph factorization," in Proceedings of the 22Nd International Conference on World Wide Web, WWW'13, (New York, NY, USA), pp. 37-48, ACM, 2013.

[21] B. W. Kernighan and S. Lin, "An efficient heuristic procedure for partitioning graphs," The Bell System Technical Journal, vol. 49, pp. 291-307, Feb 1970.

[22] C. Tsourakakis, C. Gkantsidis, B. Radunovic, and M. Vojnovic, "Fennel: Streaming graph partitioning for massive scale graphs," in Proceedings of the 7th ACM International Conference on Web Search and Data Mining, WSDM '14, (New York, NY, USA), pp. 333-342, ACM, 2014.

[23] A. K. Menon and C. Elkan, "Link prediction via matrix factorization," in Proceedings of the 2011 European Conference on Machine Learning and Knowledge Discovery in Databases - Volume Part II, ECML PKDD'11, (Berlin, Heidelberg), pp. 437-452, Springer-Verlag, 2011.

[24] Y. Zhang, M. Zhang, Y. Liu, S. Ma, and S. Feng, "Localized matrix factorization for 
recommendation based on matrix block diagonal forms," in Proceedings of the 22Nd International Conference on World Wide Web, WWW'13, (New York, NY, USA), pp. 1511-1520, ACM, 2013.

[25] A. Grover and J. Leskovec, "node2vec: Scalable feature learning for networks," CoRR, vol. abs/1607.00653, 2016.

[26] S. Cao, W. Lu, and Q. Xu, "Deep neural networks for learning graph representations," in Thirtieth AAAI Conference on Artificial Intelligence, 2016.

[27] J. Tang, M. Qu, M. Wang, M. Zhang, J. Yan, and Q. Mei, "Line: Large-scale information network embedding.," in $W W W$, ACM, 2015.

[28] K. D. Bollacker, C. Evans, P. Paritosh, T. Sturge, and J. Taylor, "Freebase: a collaboratively created graph database for structuring human knowledge," in SIGMOD Conference, 2008.

[29] G. A. Miller, "Wordnet: A lexical database for english," Commun. ACM, vol. 38, pp. 39-41, Nov. 1995.

[30] J. Lehmann, R. Isele, M. Jakob, A. Jentzsch, D. Kontokostas, P. N. Mendes, S. Hellmann, M. Morsey, P. van Kleef, S. Auer, and C. Bizer, "Dbpedia - a largescale, multilingual knowledge base extracted from wikipedia," Semantic Web, vol. 6, pp. 167-195, 2015.

[31] F. M. Suchanek, G. Kasneci, and G. Weikum, "Yago: a core of semantic knowledge," in Proceedings of the 16th international conference on World Wide Web, pp. 697-706, ACM, 2007.

[32] A. Carlson, J. Betteridge, B. Kisiel, B. Settles, E. R. Hruschka, Jr., and T. M. Mitchell, "Toward an architecture for never-ending language learning," in Proceedings of the Twenty-Fourth AAAI Conference on Artificial Intelligence, AAAI'10, pp. 1306-1313, AAAI Press, 2010.

[33] R. Hoffmann, C. Zhang, X. Ling, L. Zettlemoyer, and D. S. Weld, "Knowledge-based weak supervision for information extraction of overlapping relations," in Proceedings of the 49th Annual Meeting of the Association for Computational Linguistics: Human Language Technologies-Volume 1, pp. 541-550, Association for Computational Linguistics, 2011.

[34] J. Daiber, M. Jakob, C. Hokamp, and P. N. Mendes, "Improving efficiency and accuracy in multilingual entity extraction," in Proceedings of the 9th International Conference on Semantic Systems, pp. 121-124, ACM, 2013.

[35] A. Bordes, J. Weston, and N. Usunier, "Open question answering with weakly supervised embedding models," in Joint European Conference on Machine Learning and Knowledge Discovery in Databases, pp. 165-180, Springer, 2014. 
[36] A. Bordes, S. Chopra, and J. Weston, "Question answering with subgraph embeddings," arXiv preprint arXiv:1406.3676, 2014.

[37] Q. Wang, Z. Mao, B. Wang, and L. Guo, "Knowledge graph embedding: A survey of approaches and applications," IEEE Transactions on Knowledge and Data Engineering, vol. 29, no. 12, pp. 2724-2743, 2017.

[38] A. Bordes, N. Usunier, A. Garcia-Duran, J. Weston, and O. Yakhnenko, “Translating embeddings for modeling multi-relational data," in Advances in neural information processing systems, pp. 2787-2795, 2013.

[39] Y. Lin, Z. Liu, M. Sun, Y. Liu, and X. Zhu, "Learning entity and relation embeddings for knowledge graph completion," in Proceedings of the Twenty-Ninth AAAI Conference on Artificial Intelligence, AAAI'15, pp. 2181-2187, AAAI Press, 2015.

[40] Y. Lin, Z. Liu, H. Luan, M. Sun, S. Rao, and S. Liu, "Modeling relation paths for representation learning of knowledge bases," arXiv preprint arXiv:1506.00379, 2015.

[41] Z. Wang, J. Zhang, J. Feng, and Z. Chen, "Knowledge graph embedding by translating on hyperplanes.," in AAAI, pp. 1112-1119, 2014.

[42] C. Zhang, M. Zhou, X. Han, Z. Hu, and Y. Ji, "Knowledge graph embedding for hyper-relational data," Tsinghua Science and Technology, vol. 22, pp. 185-197, April 2017.

[43] K. Guu, J. Miller, and P. Liang, "Traversing knowledge graphs in vector space," arXiv preprint arXiv:1506.01094, 2015.

[44] M. Nickel, L. Rosasco, and T. A. Poggio, "Holographic embeddings of knowledge graphs," CoRR, vol. abs/1510.04935, 2015.

[45] P. J. Werbos, "Backpropagation through time: what it does and how to do it," Proceedings of the IEEE, vol. 78, no. 10, pp. 1550-1560, 1990.

[46] A. Bordes, J. Weston, R. Collobert, and Y. Bengio, "Learning structured embeddings of knowledge bases," in Twenty-Fifth AAAI Conference on Artificial Intelligence, 2011.

[47] K. Toutanova, V. Lin, W.-t. Yih, H. Poon, and C. Quirk, "Compositional learning of embeddings for relation paths in knowledge base and text," in Proceedings of the 54th Annual Meeting of the Association for Computational Linguistics (Volume 1: Long Papers), vol. 1, pp. 1434-1444, 2016.

[48] M. Nickel, V. Tresp, and H.-P. Kriegel, "Factorizing yago: scalable machine learning for linked data," in Proceedings of the 21st international conference on World Wide Web, pp. 271-280, ACM, 2012.

[49] R. Hoffmann, C. Zhang, X. Ling, L. Zettlemoyer, and D. S. Weld, "Knowledge-based 
weak supervision for information extraction of overlapping relations," in Proceedings of the 49th Annual Meeting of the Association for Computational Linguistics: Human Language Technologies-Volume 1, pp. 541-550, Association for Computational Linguistics, 2011.

[50] A. Guerrieri and A. Montresor, "Distributed edge partitioning for graph processing," CoRR, vol. abs/1403.6270, 2014.

[51] K. Andreev and H. Räcke, "Balanced graph partitioning," in Proceedings of the Sixteenth Annual ACM Symposium on Parallelism in Algorithms and Architectures, SPAA '04, (New York, NY, USA), pp. 120-124, ACM, 2004.

[52] J. Leskovec, J. Kleinberg, and C. Faloutsos, "Graph evolution: Densification and shrinking diameters," ACM Trans. Knowl. Discov. Data, vol. 1, Mar. 2007.

[53] L. Tang and H. Liu, "Leveraging social media networks for classification," Data Mining and Knowledge Discovery, vol. 23, pp. 447-478, Nov 2011.

[54] J. Scott, Social network analysis. Sage, 2017.

[55] L. C. Freeman, Research methods in social network analysis. Routledge, 2017.

[56] M. A. Ferrag, L. Maglaras, and A. Ahmim, "Privacy-preserving schemes for ad hoc social networks: A survey," IEEE Communications Surveys \& Tutorials, vol. 19, no. 4, pp. 3015-3045, 2017.

[57] J. Kim and M. Hastak, "Social network analysis: Characteristics of online social networks after a disaster," International Journal of Information Management, vol. 38, no. 1, pp. 86-96, 2018.

[58] X. Wang, F. Wei, X. Liu, M. Zhou, and M. Zhang, "Topic sentiment analysis in twitter: a graph-based hashtag sentiment classification approach," in Proceedings of the 20th ACM international conference on Information and knowledge management, pp. 1031-1040, ACM, 2011.

[59] L. Page, S. Brin, R. Motwani, and T. Winograd, "The pagerank citation ranking: Bringing order to the web.," Technical Report 1999-66, Stanford InfoLab, November 1999. Previous number $=$ SIDL-WP-1999-0120.

[60] W. Liu, H. Li, and B. Xie, "Real-time graph partition and embedding of large network," in 2018 18th IEEE/ACM International Symposium on Cluster, Cloud and Grid Computing (CCGRID), pp. 432-441, May 2018.

[61] T. A. Plate, "Holographic reduced representations," IEEE Transactions on Neural networks, vol. 6, no. 3, pp. 623-641, 1995.

[62] R. Socher, D. Chen, C. D. Manning, and A. Ng, "Reasoning with neural tensor networks for knowledge base completion," in Advances in neural information processing systems, pp. 926-934, 2013. 
[63] T. Mikolov, K. Chen, G. Corrado, and J. Dean, "Efficient estimation of word representations in vector space," arXiv preprint arXiv:1301.3781, 2013. 


\section{CURRICULUM VITAE}

NAME: $\quad$ Wenqi Liu

ADDRESS: $\quad$ W.S. Speed Hall

University of Louisville

Louisville, KY 40208

EDUCATION: $\quad$ Ph.D. Electrical Engineering, University of Louisville, expected in 2019

M.S. Compute Engineering, Stevens Institute of Technology, 2013

B.S. Electrical Engineering, Donghua University, 2011

RESEARCH: $\quad$ Wireless Communication, Representation Learning, Knowledge Graph

Large-scale Network, Graph Embedding, Distributed Computing

TEACHING: $\quad$ ECE 322 Introduction to the Computation Tools

ECE 550 Communication and Modulation - GTA

ECE 252 Introduction to Electrical Engineering - GTA

\section{PUBLICATION:}

[1] Wenqi Liu, Hongxiang Li, Bin Xie, "Real-time Distributed Graph Partition and Embedding of Large Network" 2018 IEEE/ACM International Symposium on Cluster, Cloud and Grid Computing (CCGRID), Washington, DC, 2018, pp. 432-441.

[2] Wenqi Liu, Hongxiang Li, Bin Xie, "Optimize the Spectrum Sensing and Decision Making Strategies under Uncertainty for SATCOM" IEEE MILCOM, Baltimore, MD, 2016, pp. 168-173.

[3] Xiaohui Zhang, Hongxiang Li, Wenqi Liu, "Iterative IQ Imbalance Compensation Receiver for Single Carrier Transmission," IEEE Transactions on Vehicular Technology, 
vol. 66, no. 9, pp. 8238-8248, Sept. 2017.

[4] Chen Cao, Hongxiang Li, Zixia Hu, Wenqi Liu and Xiaohui Zhang, "Physical-Layer Secrecy Performance in Finite Blocklength Case," 2015 IEEE Global Communications Conference (GLOBECOM), pp. 1-6, San Diego, CA, 2015.

[5] Wenqi Liu, "Fuzzy Synthetic Evaluation on the Campus Network," Technology Wind Journal, ISSN1671-7341, China, 2011.

[6] Wenqi Liu, "Study on the current status and development tendency of Chinese retail chains industry," Charming China , ISSN1673-0992, Dec, 2010. 